\title{
Astronomy in Antarctica
}

\author{
Michael G. Burton
}

Received: 01/05/10 / Accepted: 10/06/10

\begin{abstract}
Antarctica provides a unique environment for astronomers to practice their trade. The cold, dry and stable air found above the high Antarctic plateau, as well as the pure ice below, offers new opportunities for the conduct of observational astronomy across both the photon and the particle spectrum. The summits of the Antarctic plateau provide the best seeing conditions, the darkest skies and the most transparent atmosphere of any earth-based observing site. Astronomical activities are now underway at four plateau sites: the AmundsenScott South Pole Station, Concordia Station at Dome C, Kunlun Station at Dome A and Fuji Station at Dome F, in addition to long duration ballooning from the coastal station of McMurdo, at stations run by the USA, France / Italy, China, Japan and the USA, respectively. The astronomy conducted from Antarctica includes optical, infrared, terahertz and sub-millimetre astronomy, measurements of cosmic microwave background anisotropies, solar astronomy, as well as high energy astrophysics involving the measurement of cosmic rays, gamma rays and neutrinos. Antarctica is also the richest source of meteorites on our planet.
\end{abstract}

An extensive range of site testing measurements have been made over the high plateau sites. In this paper we summarise the facets of Antarctica that are driving developments in astronomy there, and review the results of the site testing experiments undertaken to quantify those characteristics of the Antarctic plateau relevant for astronomical observation. We also outline the historical development of the astronomy on the continent, and then review the principal scientific results to have emerged over the past three decades of activity in the discipline. These range from determination of the dominant frequencies of the 5 minute solar oscillation in 1979 to the highest angular scale measurements yet made of the power spectrum of the CMBR anisotropies in 2010. They span through infrared views of the galactic ecology in star formation complexes in 1999, the first clear demonstration that the Universe was flat in 2000, the first detection of polarization in the CMBR in 2002, the mapping of the warm molecular gas across the $\sim 300 \mathrm{pc}$ extent of the Central Molecular Zone of our

M.G. Burton

School of Physics, University of New South Wales, Sydney, NSW 2052, Australia

Tel.: +61-2-9385-4553

Fax: +61-2-9385-6060

E-mail: m.burton@unsw.edu.au 
Galaxy in 2003, the measurement of cosmic neutrinos in 2005, and imaging of the thermal Sunyaev Zel'dovich effect in galaxy clusters in 2008.

This review also discusses how science is conducted in Antarctica, and in particular the difficulties, as well as the advantages, faced by astronomers seeking to bring their experiments there. It also reviews some of the political issues that will be encountered, both at national and international level. Finally, the review discusses where Antarctic astronomy may be heading in the coming decade, in particular plans for infrared and terahertz astronomy, including the new facilities being considered for these wavebands at the high plateau stations.

Keywords Methods: observational - Telescopes - Site testing - Atmospheric effects · Astroparticle physics $\cdot$ Cosmic background radiation

\section{Why Astronomy in Antarctica?}

\subsection{The Antarctic Continent and its high ice plateau}

The Antarctic continent is the highest, driest and coldest of the continents on the Earth. It is the end of the Earth, literally as well metaphorically. As with all endeavours in Antarctica, it was the last continent where humans began to conduct astronomical observations from. The first astronomical discovery was made less than a century ago, and it has only been in the past two decades that major ventures in the discipline have taken place. Yet, with current technology, astronomy is no longer difficult to undertake in Antarctica, given appropriate resources and fore-planning. Moreover, the potential Antarctica offers for furthering a wide and diverse range of frontier investigations in astronomy is unmatched, in comparison with any other location on our planet.

Antarctica is the fifth largest continent, with a land area of 14 square million kilometres. The amount of exposed land is, however, tiny, about $2 \%$ of the total and confined almost entirely to the coastal fringe. As a continent, Antarctica is dominated by ice, with the area covered virtually doubling between the summer and winter extremes; the ice sheets extend up to one thousand kilometres over the Southern Ocean from the coast at their September peak. From an astronomer's perspective it is the ice mass of the Antarctic plateau that draws the attention. For, while the continent is crossed by one of the world's great mountain ranges, the Trans Antarctic Mountains that stretch nearly 5,000 km from the Weddell Sea to the Ross Sea, all but its highest peaks (the nunataks) are obscured from view, buried under the ice sheet that makes up the Antarctic plateau.

The land itself lies under up to four kilometres of ice, with the ice surface very gradually rising from the coast, over a distance of several hundred kilometres, to reach over 4,000 $\mathrm{m}$ at Dome A (though the peaks of some of the mountains rise higher than this, for instance the Vinson Massif in West Antarctica is 4,897 m high). The area of ice over 3,000 m elevation is almost as large as the continent of Australia. This is the Antarctic plateau. Its great extent makes Antarctica the highest continent, as measured by average elevation. It also contains the coldest and driest regions of our planet. A temperature of $-90^{\circ} \mathrm{C}$ was once measured at the Russian Vostok station, the lowest ever recorded, and winters average $-60^{\circ} \mathrm{C}$ over the plateau. Typical precipitable water vapour levels of $250 \mu \mathrm{m}$ exist for much of the year and levels can fall below $100 \mu \mathrm{m}$ in places at times, the driest air on Earth.

Of crucial importance for the conduct of astronomy is that there is little wind on top of the plateau. The Antarctic atmospheric circulation pattern centres about the South Pole, and 
the dominant airflow is a slow settling from the stratosphere, to feed a steady, downwardflowing wind off the plateau. This wind is katabatic in origin, starting from the highest points and picking up speed as it falls towards the coast, under gravity. With an average slope of about one tenth of a degree, the wind is also gentle. Over the highest parts of the plateau typical wind speeds are only $1-2 \mathrm{~m} / \mathrm{s}$. Though, as it nears the coast and the gradient increases, the wind speed picks up - and can lead to the ferocious storms that are a part of Antarctic folklore.

The debilitating effects of the atmosphere are largely confined to a thin turbulent surface boundary layer. In winter this can be only a few metres thick over the summits of the plateau. Above it, the seeing is exceptionally low. So too is the level of scintillation noise, and both the isoplanatic angle and coherence time are large, in comparison with the best temperate latitude sites. These facets provide conditions than can yield extraordinary image clarity and stability for a wide range astronomical observations. As these conditions have been quantified over the past decade, and their implications understood, they have drawn the interest of astronomers to the continent for its potential to provide superlative observatory sites.

\subsection{This Review}

This paper provides an overview of the subject of astronomy in Antarctica. We describe the features of the continent of special interest to astronomers, and the sites under consideration for observatories. We also summarise the principal results obtained from the extensive program of site testing of the Antarctic plateau that has taken place over the past two decades. A brief history of the development of astronomy in Antarctica is given, followed by examples of some of the science obtained. As will be apparent, this includes a diverse range of fields as well as techniques. A section discusses how science is conducted in Antarctica today, and what the principal difficulties are for the investigator, in comparison to undertaking temperate-latitude astronomy. The review finishes with some personal thoughts of where astronomy will develop on the continent over the coming years. Many sources have been drawn upon for this review, and in particular it extends the earlier reviews of Storey (2005), Burton (2005) and Storey (2009). Several books have been devoted entirely to Astronomy in Antarctica, the proceedings of conferences held on the subject. They include the proceedings of the American Institute of Physics conference on Astronomy in Antarctica in Newark, USA in 1989 (Pomerantz 1990), the Astronomical Society of the Pacific symposium on Astronomy in Antarctica in Chicago, USA in 1997 (Novak \& Landsberg, 1998), the Concordia station workshop in Capri, Italy in 2003 (Fossat \& Candidi 2003) and the three European ARENA conferences, held in Roscoff, France in 2006 (Epchtein \& Candidi 2007), Potsdam, Germany in 2007 (Zinnecker, Epchtein \& Rauer 2008) and Frascati, Italy in 2009 (Spinoglio \& Epchtein 2010).

\subsection{Sites for Astronomy in Antarctica}

The South Pole, at 2,835 m elevation, lies on the flank of the Antarctic plateau. While there are better places in Antarctica for many kinds of observation, the logistics of ready access have made the Pole the place where most of the astronomy has so far been conducted in Antarctica, at the US Amundsen-Scott South Pole station. Named after the pioneers who first reached the Pole in 1911-12, the station was established in 1957 during the International 


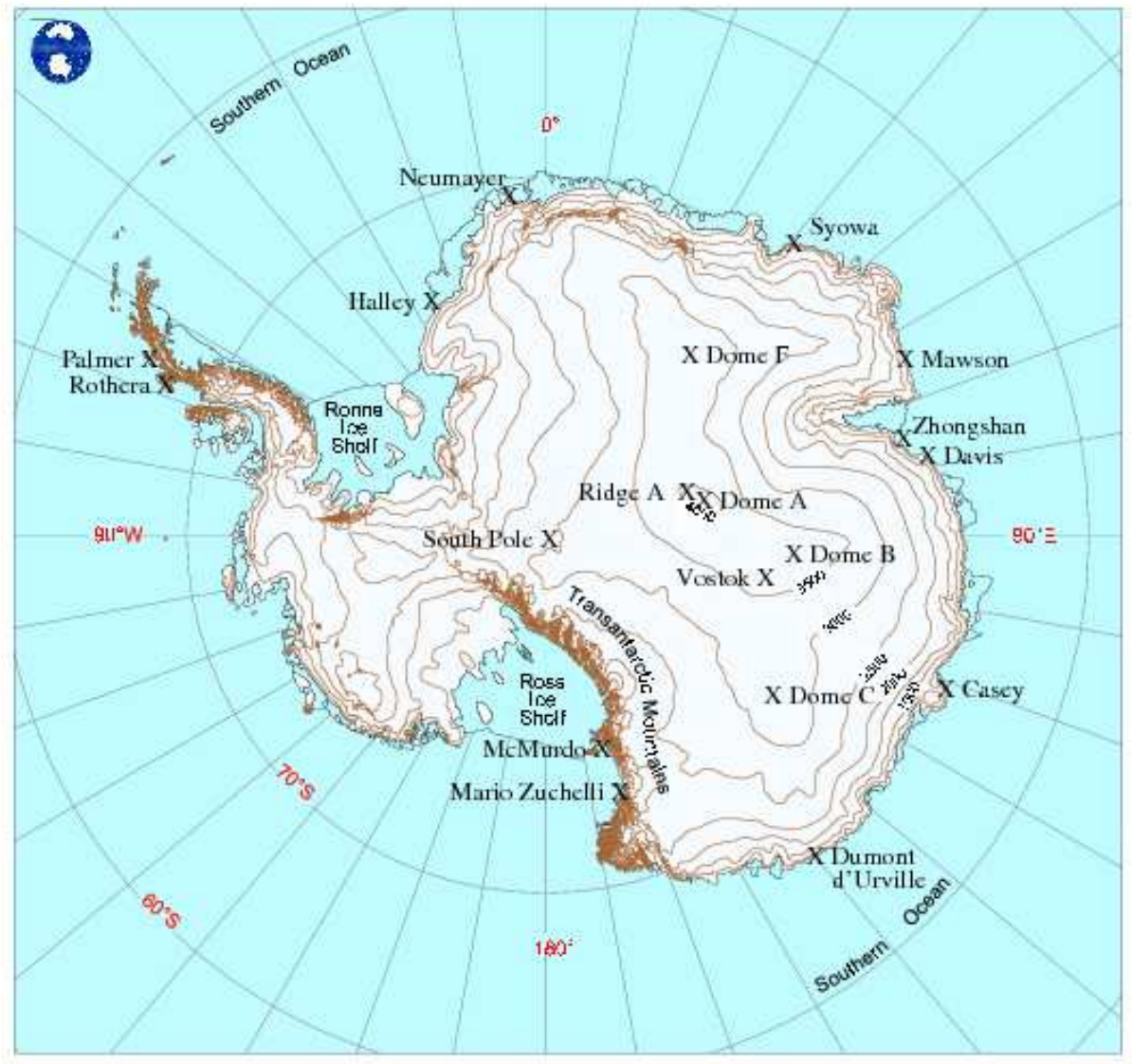

Fig. 1 Topographic map of Antarctica, with the location of the principal research stations discussed in the text indicated. The high Antarctic plateau runs along the ridge from Dome $\mathrm{F}$ to Dome $\mathrm{C}$, through Dome A, Dome B and Vostok. Ridge A lies $400 \mathrm{~km} \mathrm{SW}$ of Dome A. The South Pole lies on the flank of the Antarctic plateau. Coastal stations supporting high plateau operations are also marked; McMurdo (USA), Mario Zuchelli (Italy), Dumont d'Urville (France), Zhongshan (China) and Syowa (Japan). In addition, the locations of major coastal stations at Casey, Davis \& Mawson (Australia), Halley \& Rothera (UK), Palmer (USA) and Neumayer (Germany) are shown. Map adapted from a figure supplied by the Australian Antarctic Division, with acknowledgment.

Geophysical Year (IGY). It recently completed a major refurbishment, and supports around 60 personnel over-wintering and $\sim 250$ in the summer months. Access is via ski-equipped LC130 aircraft from McMurdo station on the coast, with daily flights over the summer period from November to February.

Concordia station at Dome C, built by France and Italy, was opened for winter operation in 2005 following a decade-long construction phase. At 3,268 m Dome $\mathrm{C}$ is one of the high 
Table 1 Astronomical Sites in Antarctica

\begin{tabular}{|c|c|c|c|c|c|c|c|}
\hline Location & $\begin{array}{l}\text { Elevation } \\
\mathrm{m}\end{array}$ & $\underset{\circ}{\text { Latitude }}$ & $\underset{\circ}{\text { Longitude }}$ & $\begin{array}{l}\text { National } \\
\text { Operator }\end{array}$ & Established & $\begin{array}{l}\text { Astronomical Applications } \\
\text { of Particular Interest }\end{array}$ & Comment \\
\hline South Pole & 2,835 & -90 & $\ldots$ & USA & 1957 & CMBR, Neutrinos, Sub-mm & $\begin{array}{l}\text { Amundsen-Scott station. } \\
\text { On flank of Antarctic plateau. }\end{array}$ \\
\hline Dome A & 4,083 & -80 & +78 & China & 2009 & $\begin{array}{l}\text { Optical, THz, IR } \\
\text { Time-series }\end{array}$ & $\begin{array}{l}\text { First visited 2005. Construction } \\
\text { of Kunlun station started } 2009 .\end{array}$ \\
\hline Dome B & 3,809 & -76 & +95 & $\ldots$ & $\ldots$ & THz, IR & Not yet visited by humans. \\
\hline Dome C & 3,268 & -75 & +123 & $\begin{array}{l}\text { France } \\
\& \text { Italy }\end{array}$ & 2005 & $\begin{array}{l}\text { Optical, Sub-mm, IR, Solar } \\
\text { Time-series, Interferometry }\end{array}$ & Concordia station. \\
\hline Dome F & 3,810 & -77 & +39 & Japan & 1995 & $\mathrm{THz}, \mathrm{IR}$ & $\begin{array}{l}\text { Fuji station, wintered (ice cores) } \\
\text { Site testing now commencing. }\end{array}$ \\
\hline Vostok & 3,488 & -78 & +107 & Russia & 1957 & Sub-mm, IR & No site quantification as yet. \\
\hline Ridge A & 4,053 & -82 & +74 & $\ldots$ & $\ldots$ & THz, IR & $\begin{array}{l}\text { Possibly 'best' site? } \\
\text { Not yet visited by humans. }\end{array}$ \\
\hline McMurdo & 0 & -78 & +167 & USA & 1956 & $\begin{array}{l}\text { Long Duration Ballooning } \\
\text { (CMBR, THz, sub-mm, Solar, } \\
\text { neutrinos), Cosmic Rays }\end{array}$ & $\begin{array}{l}\text { Coastal station hosting } \\
\text { the LDBF. }\end{array}$ \\
\hline Mawson & 0 & -68 & +63 & Australia & 1954 & Cosmic Rays & Coastal station. \\
\hline
\end{tabular}

This table summarises the sites in Antarctica where astronomy is conducted or is under consideration, including the applications of particular interest at individual sites.

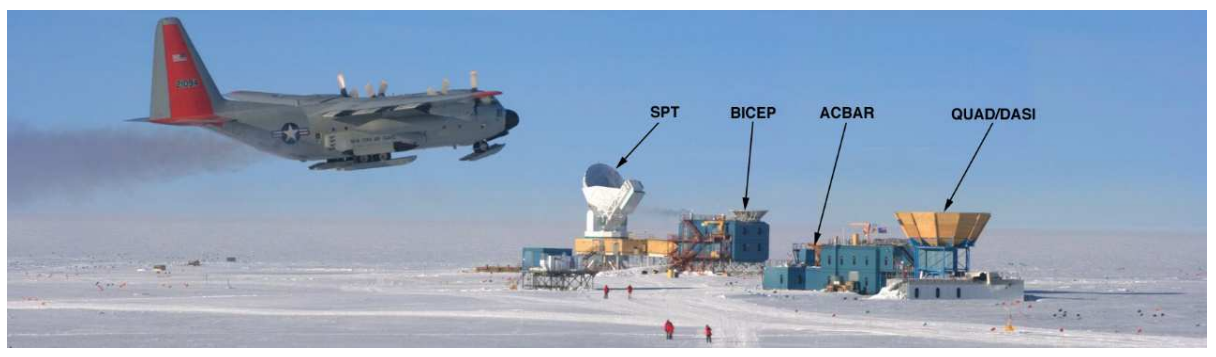

Fig. 2 The Martin A Pomerantz Observatory (MAPO) in the "Dark Sector" of the US Amundsen-Scott South Pole Station. From left to right, the telescopes are the $10 \mathrm{~m}$ South Pole Telescope (SPT), the $25 \mathrm{~cm}$ BICEP, the $2.1 \mathrm{~m}$ Viper with ACBAR and the Degree Angular Scale Interferometer (DASI) with QUaD. 44.2 provides further information on their capabilities. A ski-equipped LC130 aircraft, as used for supplying the station with all personnel and cargo, takes off from the ski-way to left. Several humans seen to foreground provide a sense of scale. They are traversing across the ski-way from the main South Pole Station base $1 \mathrm{~km}$ away (behind the camera). Image courtesy of Steffan Richter.

points of the Plateau, where the conditions are at their most stable. Extensive site quantification has taken place over the past decade, starting from before the station opened for winter operation. Plans for a range of facilities to exploit the conditions there have been developed by several European nations and by Australia. Dome $\mathrm{C}$ is accessed, for cargo, by three overland tractor traverses per season. Each traverse lasts about two weeks and originates from the coastal station of Dumont d'Urville (France). For transportation of people, Twin Otter aircraft are used, generally starting from either Dumont d'Urville or from Mario Zucchelli station (Italy).

The highest location on the plateau is the $4,083 \mathrm{~m}$ Dome A, first visited by humans in just 2005 by a Chinese team on a tractor traverse from Zhongshan station. In 2009 China began 


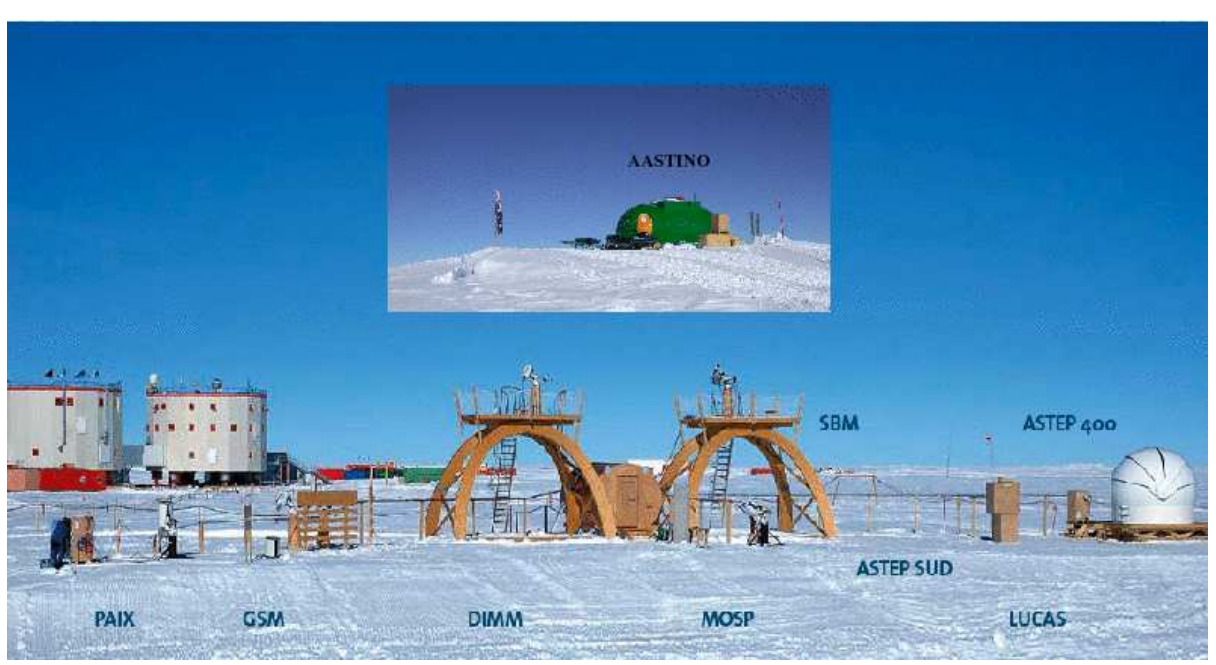

Fig. 3 Panoramic view of the French-Italian Concordia Station at Dome C, seen from the cluster of experiments that make up the Concordiastro site characterisation program. These are labelled from left to right: PAIX (photometric extinction), GSM (seeing), DIMM (seeing), MOSP (turbulence outer scale), SBM (sky brightness), ASTEP (photometry) Sud, ASTEP 400 and LUCAS (spectra of earth-shine off the Moon). The inset shows the AASTINO, the Australian automated site testing laboratory with a SODAR (sonic radar) and MASS (seeing). The twin towers of the station are seen to rear, rising $20 \mathrm{~m}$ above the ice. Credit Karim Agabi (main picture) and John Storey (for AASTINO insert).

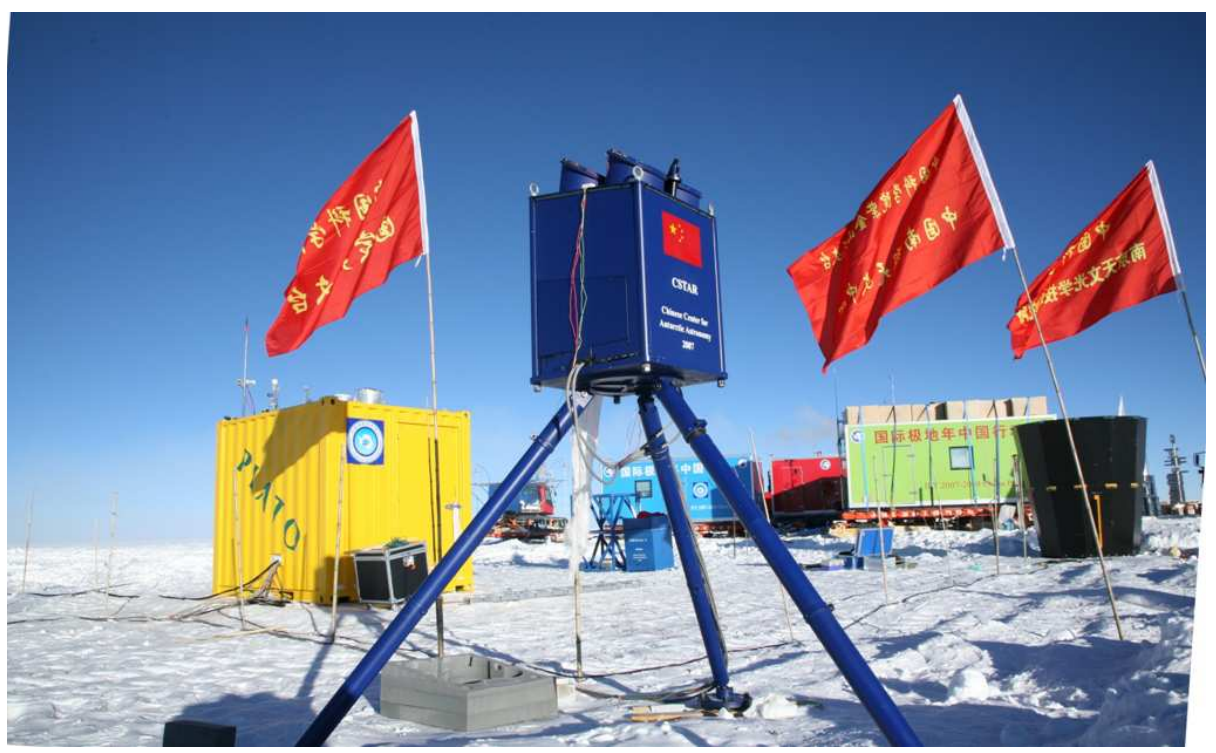

Fig. 4 The Chinese Kunlun Station at Dome A. To foreground is the CSTAR experiment (4 fixed optical telescopes pointed at the South Celestial Pole). It is controlled from the instrument module of the PLATO autonomous laboratory (yellow cabin to left). On its roof is the Gattini camera for monitoring sky brightness and cloud cover. The black cone is the SNODAR acoustic radar. The first modules of Kunlun station are to rear. Credit Zhenxi Zhu and Xu Zhou. 


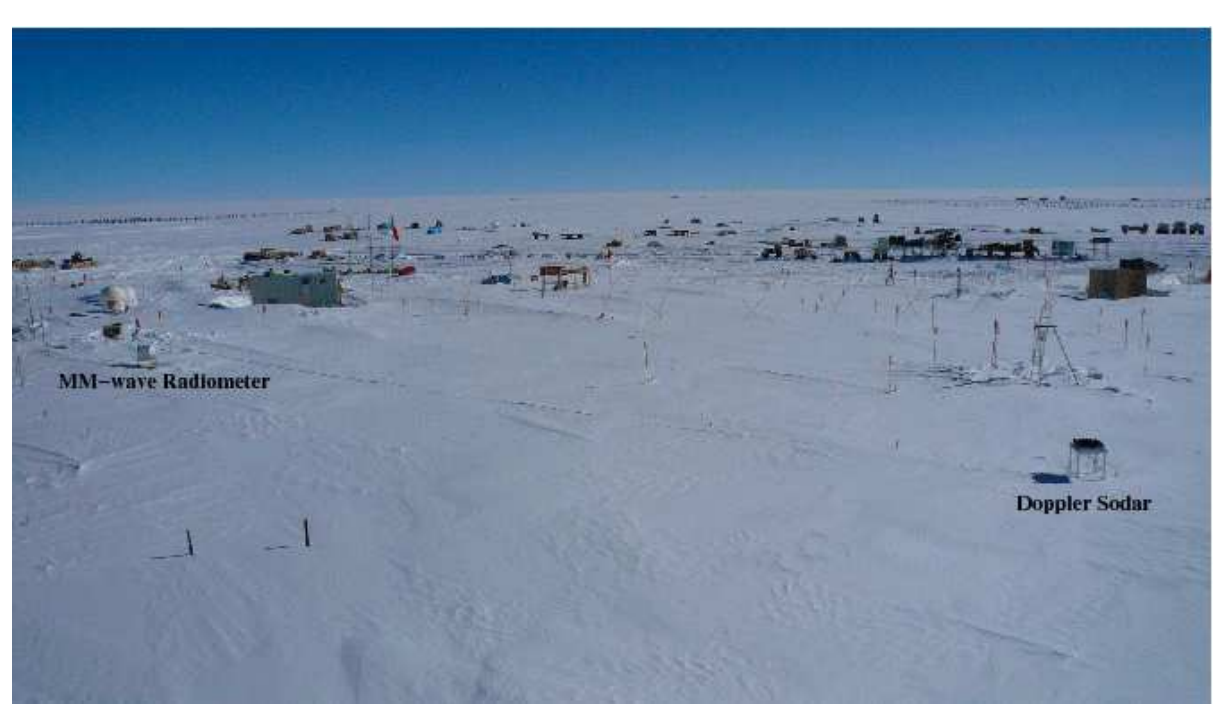

Fig. 5 The Japanese Dome Fuji station at Dome F in the 2006-07 summer, when the primary activity was ice core drilling. Two site testing experiments are indicated; a mm-wave radiometer for measuring sky transparency and a Doppler sodar for boundary layer turbulence. Credit Hideaki Motoyama, National Institute Polar Research, Japan.

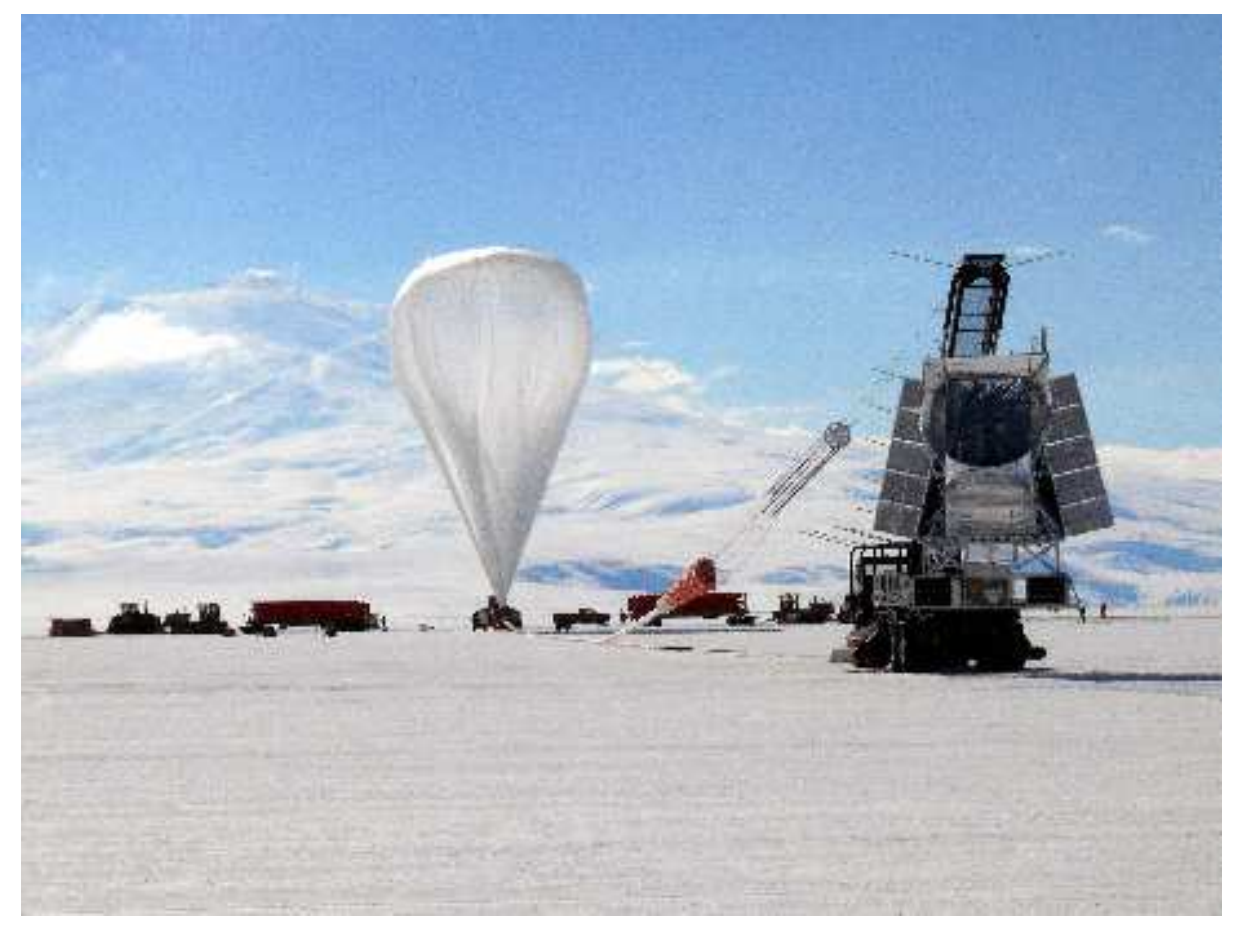

Fig. 6 Launch of the $2 \mathrm{~m}$ BLAST sub-millimetre telescope from the Long Duration Balloon Facility (LDBF) on Williams air field at the US coastal station of McMurdo in December 2006. The 3,800 m volcano Mount Erebus is in the background. Credit: Mark Halpern. 
Table 2 Typical gains achievable from an ice Dome of the Antarctic plateau compared to excellent temperate latitude sites (adapted from Storey, 2009), together with a synopsis of the applications that are facilitated.

\begin{tabular}{|c|c|c|}
\hline Parameter & $\begin{array}{l}\text { Antarctic } \\
\text { Advantage }\end{array}$ & Astronomical Consequence \\
\hline $\begin{array}{l}\text { Atmospheric Seeing } \\
\text { (above } 10-40 \mathrm{~m} \text { Boundary Layer) } \\
\text { Isoplanatic Angle }\end{array}$ & $\begin{array}{l}2-3 \times \text { better } \\
2-3 \times \text { larger }\end{array}$ & $\begin{array}{l}\text { Better spatial resolution } \\
\text { Better point source sensitivity } \\
\text { Better Adaptive Optics sky coverage }\end{array}$ \\
\hline Coherence Time & $2.5 \times$ longer & $\begin{array}{l}\text { Increased sensitivity for } \\
\text { Adaptive Optics \& Interferometry }\end{array}$ \\
\hline Scintillation Noise & $3-4 \times$ less & $\begin{array}{l}\text { High dynamic range imaging } \\
\text { More precise photometry }\end{array}$ \\
\hline IR sky background & $20-100 \times$ less & $\begin{array}{l}\text { Increased infrared sensitivity } \\
\text { Improved photometric stability }\end{array}$ \\
\hline Aerosols & up to $50 \times$ lower & $\begin{array}{l}\text { Better infrared windows } \\
\text { Improved sky stability }\end{array}$ \\
\hline Water Vapour & $3-5 \times$ lower & New windows from IR to sub-mm \\
\hline Ice & Vast quantities! & $\begin{array}{l}\text { Neutrino detectors } \\
\text { Paleoclimate }\end{array}$ \\
\hline
\end{tabular}

the construction of Kunlun station at the site. Already several astronomical experiments have been conducted here, making use of an Australian robotic observatory. Ambitious plans are being formulated for IR and $\mathrm{THz}$ telescopes within the next decade. Currently one traverse is conducted each summer season, carrying all people and $\sim 570$ tonnes of equipment. No winter-overs have yet been conducted.

There are other high plateau sites where astronomy could be conducted. These include the Russian station of Vostok $(3,488 \mathrm{~m})$ and the Japanese station at Dome F $(3,810 \mathrm{~m})$, both of which have stations that have been used for wintering. Site testing is being initiated at Dome F. Another ice dome, Dome B (3,809 m), lies on the SE end of the ridge from Dome A, before the plateau drops towards Vostok. Ridge A $(4,053 \mathrm{~m})$, lying at the end of a ridge running SW from Dome A, has also been proposed as a site where conditions may even be superior to Dome A for some applications.

Not all Antarctic astronomy needs to be done on the plateau, however, in particular cosmic-ray observations. Facilities for such measurements exist at the coastal stations of Mawson (Australia) and McMurdo (USA). Neutron monitors also operate at several coastal locations. In addition, McMurdo houses a long-duration balloon facility, taking advantage of the high-altitude circumpolar winds which permit balloons to remain aloft for periods of 10-30 days at times during summer, circling the continent as they do so.

A map showing these plateau sites, together with some of the coastal sites associated with their logistic support, is shown in Fig. 1 Images of the four plateau stations, together with the McMurdo Long Duration Balloon Facility, are shown in Figs. 26 Table 1 summarises these sites and lists the astronomical applications currently of interest at each. Access to the continent is by both air and sea, and from there to the plateau by ski-equipped planes and/or overland traverse, depending on the logistic capabilities of the participating nations. 
Table 3 Astronomical Techniques for Antarctica

\begin{tabular}{ll}
\hline Technique & Advantage \\
\hline Optical & Imaging quality $\left(\varepsilon_{0}\right)$; exceptional seeing above shallow surface boundary layer \\
Infrared & $\begin{array}{l}\text { Low sky \& telescope background } \\
\text { Sky flux stability } \\
\text { Image quality }\end{array}$ \\
THz & Opens the window \\
Sub-millimetre & $\begin{array}{l}\text { Improved windows } \\
\text { Sky stability }\end{array}$ \\
Millimetre (CMBR) & Sky stability \\
Time-series & High duty-cycle measurements \\
& Stability \\
Precision Photometry & Long, uninterrupted periods of darkness \\
Low scintillation noise \\
IRterferometry + & Improved values for $\tau_{0}, \theta_{0} \& r_{0}$ and Coherence Volume $\left(\theta^{2} \tau_{0} / \varepsilon^{2}\right)$ \\
Cosmic Rays & L Temperature-stability for delay lines \\
Neutrinos & Low energy threshold (proximity to geomagnetic pole) \\
Balloons & Pure ice as an absorber \\
Solar & Long duration flights in constant environment \\
\hline This table summarises astronomical techniques and fields where Antarctica can provide special advantages \\
over other earth-based observatory locations, and the nature of that advantage.
\end{tabular}

\section{Site Conditions for Astronomical Observations from the Antarctica Plateau}

Two primary factors have driven much of the current interest in Antarctic astronomy - the coldest and driest conditions on Earth that are found in the air above the Antarctic plateau. The cold reduces background fluxes in the infrared. Over infrared to sub-millimetre wavelengths the dry air results in many windows in the atmosphere opening up to observation. A host of secondary factors provide additional reasons for astronomers to conduct observations from Antarctica (see also Tables 2 \& 3). These include the stability of the atmosphere and its thin surface boundary layer, low levels of pollution and dust aerosols in the air, high cloud-free fractions, the ability to conduct continuous or long-duration monitoring, increased low-energy cosmic-ray fluxes arising from the proximity to the magnetic pole, low levels of seismic activity and the vast quantities of pure ice available as an absorber of particles. Some of these secondary factors have proven to be particularly potent. For instance, the ice is being used to construct a neutrino detector at the South Pole with a cubic kilometre of collecting volume (IceCube). The confinement of most of the turbulence in the air to a thin layer just above the ice, over the summits of the plateau, creates conditions that are particularly favourable for wavefront correction of light, as well as providing telescopes with extraordinary good seeing if they were to be raised beyond the boundary layer.

\subsection{Infrared Sky Background: the coldest locations on Earth}

The temperature drop from freezing point to $-60^{\circ} \mathrm{C}$, about the change going from Mauna Kea in Hawaii to the South Pole in winter, would lead to a fall in the sky background of 200 times at $2.4 \mu \mathrm{m}$, if the sky were thermally emitting as a blackbody. The realisation that this drop could open a new window for deep cosmological studies drove initial efforts to quantify the infrared sky emission in Antarctica (Harper 1990, Burton, Allen \& McGregor 1993). Subsequent measurements found the drop to be a factor of $\sim 50$ at the South Pole, being 


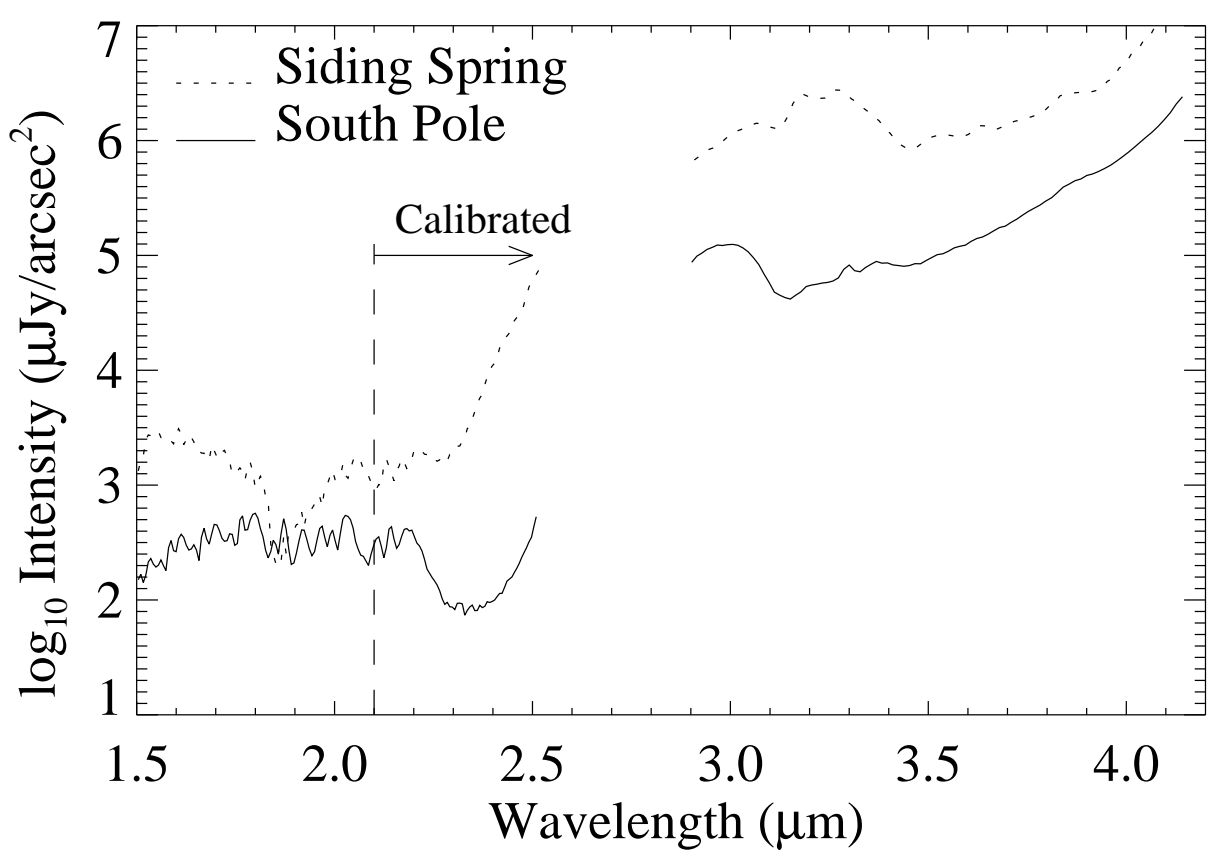

Fig. 7 The near-IR sky emission, in $\mu \mathrm{Jy} \operatorname{arcsec}^{-2}$, above the South Pole in winter, from 1.5-4 $\mu \mathrm{m}$, as measured by the IRPS instrument (Phillips et al. 1999), and compared to a similar spectrum obtained from Siding Spring Observatory in Australia. The sharp drop in flux between $2.27-2.45 \mu \mathrm{m}$ is the so-called 'cosmological window'. From $2.2 \mu \mathrm{m}$ longwards the sky is between 20 and 100 times darker than the temperate latitude site. Note that the data only have a nominal calibration factor applied shortward of $2.1 \mu \mathrm{m}$.

limited by residual high-altitude airglow emission (Nguyen et al. 1996, Ashley et al. 1996; see Fig. 77. However, from 3-30 $\mu \mathrm{m}$ the sky background was also found to be 10-20 times darker than at the best temperate latitude sites (see Fig. 8, Smith \& Harper 1998, Phillips et al. 1999, Chamberlain et al. 2000, Lawrence 2004). Such gains at mid-IR wavelengths were unexpected, for the drop in the blackbody flux near its peak emission wavelength is only a factor of 2-3 for the fall in temperature. It turned out that in the mid-IR the emission from aerosols also dropped significantly, with only ice crystals contributing to the emission, and not the dust found at temperate sites. Longwards of $20 \mu \mathrm{m}$ the drop in background is less, and arises principally from the reduced water vapour in the air, lowering the emissivity of the atmospheric windows.

Only summer (i.e. day time) thermal IR measurements have so far been obtained from a high plateau site (Dome C; Walden et al. 2005). However the sky fluxes from 3-20 $\mu$ m were found to be comparable with those measured at the South Pole in winter, as well as being exceptionally stable. Table 4 summarises the sky backgrounds at Dome $\mathrm{C}$ inferred from all these measurements and modelling, as listed in Burton et al. (2005).

\subsection{Atmospheric Transparency: the driest locations on Earth}

The amount of precipitable water vapour in the atmosphere determines the quality of a site from longwards of $20 \mu \mathrm{m}$ to the millimetre regime, as well as having a substantial bearing 
Table 4 Comparison of Sky Fluxes at Dome C, Antarctica to Mauna Kea, Hawaii

\begin{tabular}{cccc}
\hline Band & $\begin{array}{c}\text { Wavelength } \\
\mu \mathrm{m}\end{array}$ & $\begin{array}{c}\text { Mauna Kea } \\
\text { Jy arcsec }^{-2}\end{array}$ & $\begin{array}{c}\text { Dome C } \\
\text { V }\end{array}$ \\
\hline V.55 & $6(-6)$ & $6(-6)$ \\
$\mathrm{R}$ & 0.65 & $1(-5)$ & $1(-5)$ \\
$\mathrm{I}$ & 0.80 & $2(-5)$ & $2(-5)$ \\
$\mathrm{J}$ & 1.21 & $9(-4)$ & $5(-4)$ \\
$\mathrm{H}$ & 1.65 & $3(-3)$ & $1(-3)$ \\
$\mathrm{K}^{\prime}$ & 2.16 & $2(-3)$ & $\ldots$ \\
$\mathrm{K}_{\text {dark }}$ & 2.30 & $\ldots$ & $1(-4)$ \\
$\mathrm{L}$ & 3.76 & $2(+0)$ & $2(-1)$ \\
$\mathrm{M}$ & 4.66 & $4(+1)$ & $5(-1)$ \\
$\mathrm{N}$ & 11.5 & $2(+2)$ & $2(+1)$ \\
$\mathrm{Q}$ & 20 & $3(+3)$ & $5(+2)$ \\
\hline
\end{tabular}

These fluxes are derived from a combination of measurements and modelling, as listed in Burton et al. 2005. For the $\mathrm{K}$-band we compare $\mathrm{K}^{\prime}$ to $\mathrm{K}_{\text {dark }}$ as these are the parts of the window where the deepest observations may be made at Mauna Kea and Dome C, respectively. Note that $6(-6) \equiv 6 \times 10^{-6}$ etc.

on performance from 3-20 $\mu \mathrm{m}$. At many 'good' observing sites, such as Kitt Peak in the USA and Siding Spring in Australia, typically $1 \mathrm{~cm}$ of water is found between the telescope and space. At excellent sites like Mauna Kea this falls to $1 \mathrm{~mm}$ on the best of days. On the $5,000 \mathrm{~m}$ elevation Chajnantor plateau in Chile (site of ALMA) the level occasionally reaches as low as $300 \mu \mathrm{m}$ of precipitable water vapour. At the South Pole (Chamberlin et al. 1997, Lane 1998, Peterson et al. 2003) and Dome C (Valenziano \& dall'Oglio 1999, Calisse et al. 2004, Tomasi et al. 2008) such a level is maintained for most of the year, but can fall further still.

Measurements of water vapour content over the Antarctic plateau were in fact first carried out at the Russia's Vostok station in 1972 (Burova 1986, Bromwich 1988) and from the South Pole in 1975 (Smythe \& Jackson 1977). From these and subsequent data a year round average was then determined for Pole of $450 \mu \mathrm{m} \mathrm{ppt} \mathrm{H}_{2} \mathrm{O}$ and $350 \mu \mathrm{m}$ for Vostok, with levels occasionally dropping below $100 \mu \mathrm{m}$ ppt $\mathrm{H}_{2} \mathrm{O}$ in winter. As then studied by Townes \& Melnick (1990), with such levels of atmospheric water vapour the sub-mm bands from $350 \mu \mathrm{m}$ to $1 \mathrm{~mm}$ are open virtually continuously. New bands open at times at $200 \mu \mathrm{m}-$ in the THz spectral regime. Similarly, observations from $20-30 \mu \mathrm{m}$ could be routinely made. From the highest plateau site, Dome A, windows are conjectured to appear across the 30 to $300 \mu \mathrm{m}$ range that are opaque from any other ground-based location.

The first measurements from Dome A of water vapour content have recently been made, supporting these assertions. They used a tipping radiometer operating at $450 \mu \mathrm{m}(661 \mathrm{GHz})$ on the PLATO laboratory (Saunders et al. 2009), further correlated with satellite radiometry from above (the NOAA-18 satellite observing in the $183 \mathrm{GHz} \mathrm{H}_{2} \mathrm{O}$ line). A median value of $140 \mu \mathrm{m}$ ppt $\mathrm{H}_{2} \mathrm{O}$ was found, a $25 \%$ quartile of just $100 \mu \mathrm{m}$ and the ppt $\mathrm{H}_{2} \mathrm{O}$ level fell to $25 \mu \mathrm{m}$ at its lowest, comparable to that experienced by an aircraft flying in the stratosphere (e.g. SOFIA). These conditions dramatically open up atmospheric windows across the entire infrared and sub-millimetre regimes (see Fig. 9. Hidas et al. 2000, Lawrence 2004). For instance, for $100 \mu \mathrm{m}$ of ppt $\mathrm{H}_{2} \mathrm{O}$ the atmospheric transmission is $28 \%$ at the $205 \mu \mathrm{m}$ wavelength $(1.45 \mathrm{THz})$ of the important [NII] line.

Initial summer time measurements of the atmospheric transparency have also been obtained from Dome F (Ishii et al. 2010), using a tipping radiometer operating at $220 \mathrm{GHz}$. An extremely low mean opacity of 0.045 was determined (comparable to South Pole and 


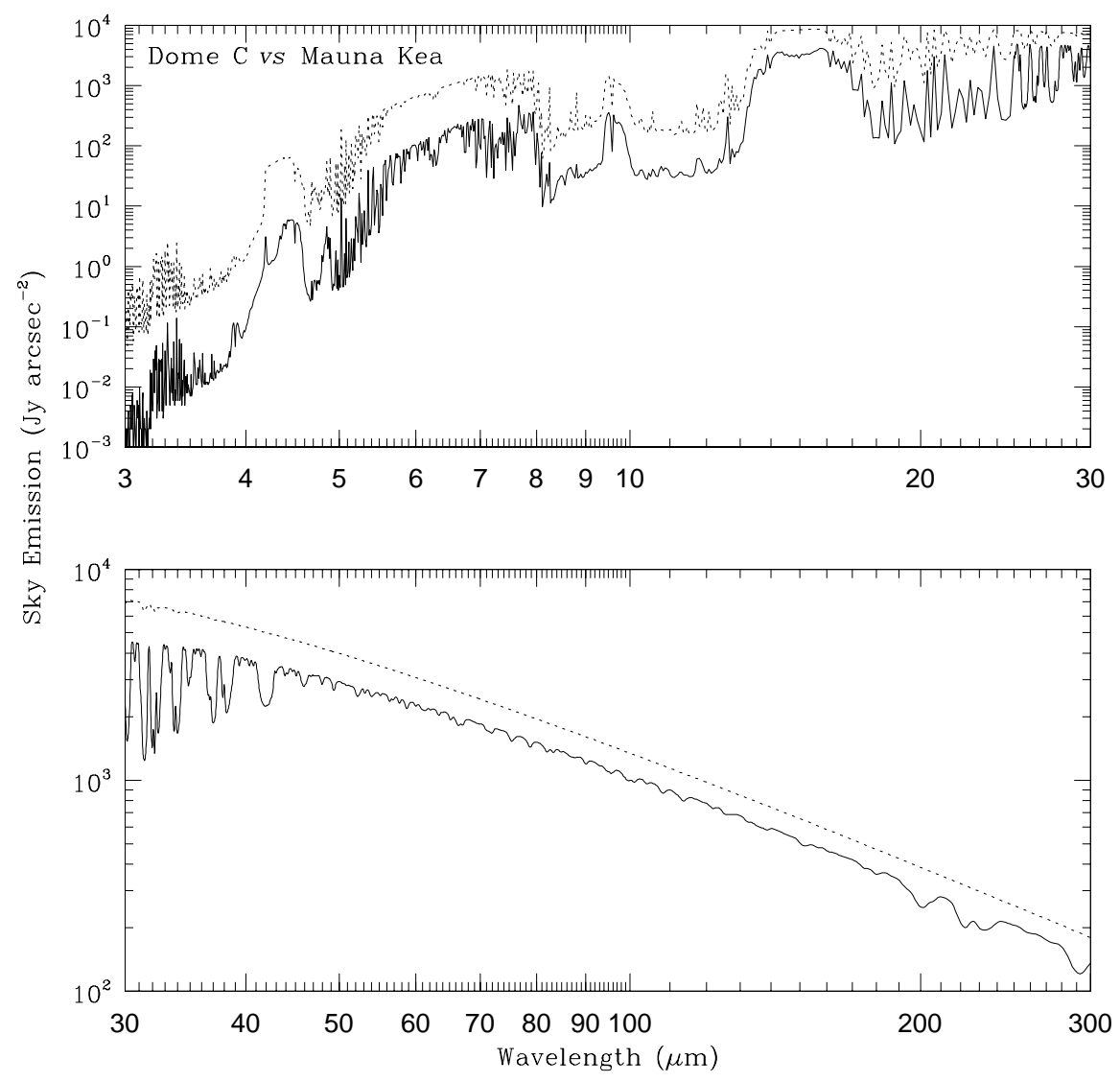

Fig. 8 Model calculations of the infrared sky emission, in $\mathrm{Jy}_{\operatorname{arcsec}}^{-2}$, above Dome C in Antarctica (solid lines; $250 \mu \mathrm{m}$ ppt $\mathrm{H}_{2} \mathrm{O}$ ), compared to Mauna Kea in Hawaii (dotted lines; $1 \mathrm{~mm}$ ppt $\mathrm{H}_{2} \mathrm{O}$ ), adapted with permission from Lawrence (2004). The upper plot shows the emission across the mid-IR (3 to $30 \mu \mathrm{m}$ ), and the lower plot the emission across the far-IR to sub-mm (30 to $300 \mu \mathrm{m})$. In the observing bands typical background reductions are between one and two orders of magnitude at the Antarctic site.

Chajnantor winter median values), and varying little over the 1 month period of the measurements. The authors model this data with $0.6 \mathrm{~mm}$ of precipitable water vapour.

There may be even better sites for astronomy on the plateau. A ridge runs south and west from Dome A and $\sim 400 \mathrm{~km} \mathrm{SW}$ is a location called "Ridge A". Examining the NOAA-18 $183 \mathrm{GHz}$ satellite measurements for this location, Yang et al. (2010) deduce even lower 25\% and $50 \%$ quartiles for the water vapour than for Dome A, of $80 \mu \mathrm{m}$ and $120 \mu \mathrm{m}$, respectively. The $158 \mu \mathrm{m}$ window $(1.9 \mathrm{THz}$ and dominant [CII] line) would even open at times, with a transmission of more than $11 \%$ for $10 \%$ of the time. Such remarkable conjectures call for in situ measurements of the water vapour content to be carried out at Ridge A to examine their veracity. 


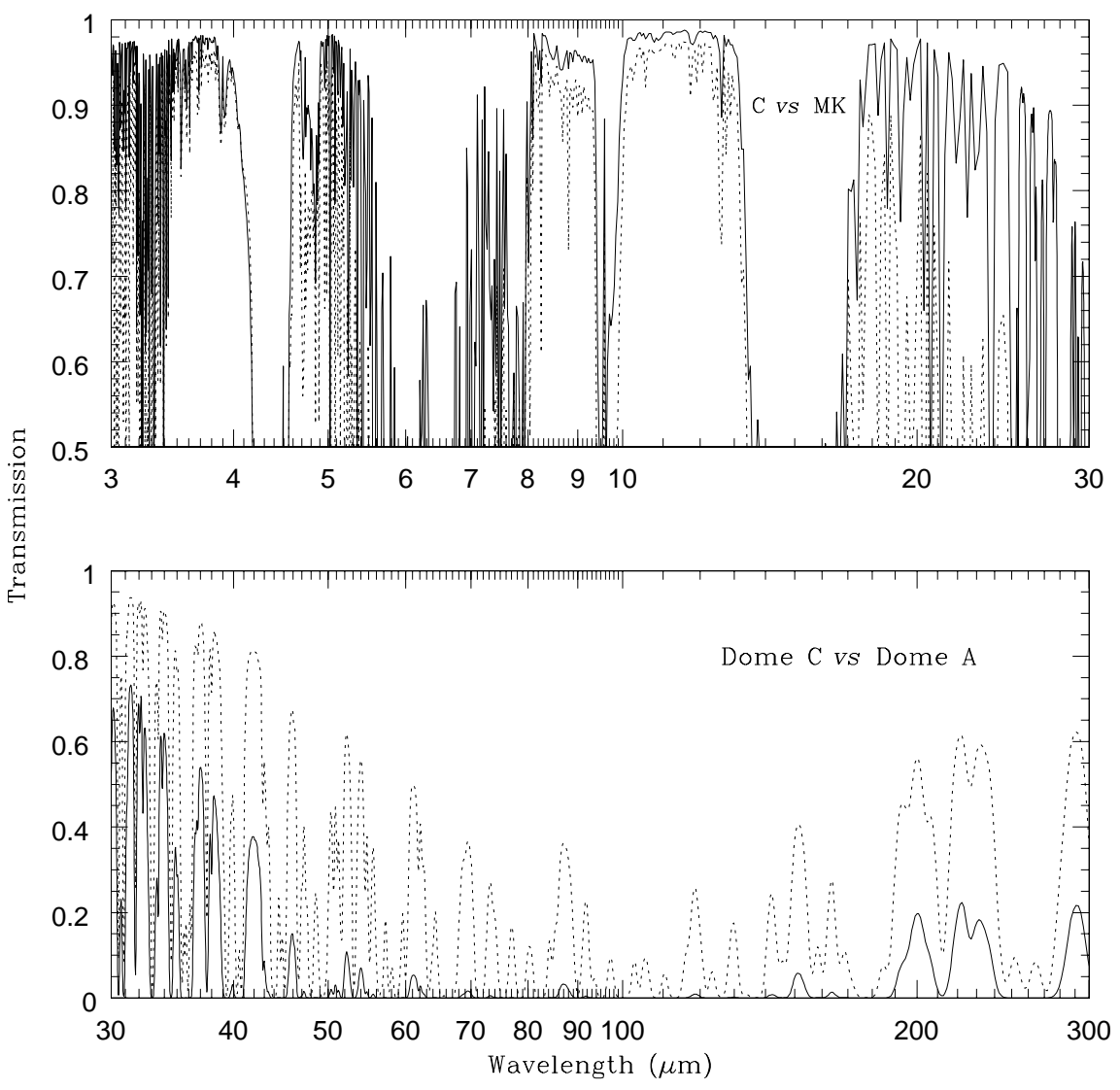

Fig. 9 Model calculations of the atmospheric transmission across the mid-IR ( 3 to $30 \mu \mathrm{m}$; upper plot), and the far-IR to sub-mm bands (30 to $300 \mu \mathrm{m}$; lower plot), adapted from Lawrence (2004). The mid-IR plot compares the transmission between typical conditions at Dome $\mathrm{C}\left(250 \mu \mathrm{m}\right.$ ppt of $\mathrm{H}_{2} \mathrm{O}$; solid line $)$ and excellent conditions at Mauna Kea (1mm ppt; dotted line), whereas in the far-IR/sub-mm plot the comparison is between average conditions at Dome C (solid) and the best conditions then predicted for Dome A $(30 \mu \mathrm{m}$; dotted), since Mauna Kea is effectively opaque in this wavelength range. Note that $25 \mu \mathrm{m} \mathrm{ppt} \mathrm{H}_{2} \mathrm{O}$ has subsequently been determined as the best value at Dome A in the analysis conducted by Yang et al. 2010.

In the near-IR, from 1-2.5 $\mu \mathrm{m}$, models by Siebenmorgen (2010) show that the average transmission at Dome $\mathrm{C}$ exceeds $40 \%$ at all wavelengths, when measured with a resolution $\mathrm{R}=250$ (there are, of course, narrow spectral regions which remain saturated). This results in a widening of the $\mathrm{J}, \mathrm{H} \& \mathrm{~K}$ bands from $1-2.5 \mu \mathrm{m}$, and of the $\mathrm{L} \& \mathrm{M}$ bands at $3.6 \& 5.0 \mu \mathrm{m}$.

2.3 Atmospheric Stability and Seeing: the thin surface boundary layer

Stability Less appreciated, but equally important for the conduct of many kinds of observation, is the stability provided by the atmosphere, particularly for measurements that can 
mitigate the effects of a thin, but turbulent, surface boundary layer. This provides for significant gains, not just in imaging quality, but also in the photometric precision that can be obtained for a measurement and for long time-series observations. In turn, these gains have implications not only for observations using conventional single-mirror telescopes but also for solar telescopes and for interferometers.

At the South Pole there is no diurnal cycle and so no corresponding daily temperature changes - simply the 6-month cycle of night and day. Any source beyond the Solar System that can be observed can also be seen continuously, and at constant zenith angle. This greatly facilitates experiments which require continuous monitoring or high duty cycles. It is also favourable for following particle cascades in air showers as a source is always seen through the same depth of atmosphere. In the IR, beyond $3 \mu \mathrm{m}$, it is perfectly feasible to observe all year round, the temperature being cold enough to facilitate day time observations. Away from the Pole there is, of course, a latitude-dependent seasonal diurnal cycle, and the length of the period of continuous darkness is reduced, but many of the advantages remain.

Not only is the strength of the sky background emission reduced in Antarctica, its level is more stable at IR and sub-mm wavelengths than at temperate sites (Smith \& Harper 1998, Peterson et al. 2003). Indeed, it is fluctuations in the level of the background (both spatially and temporally) that can provide the limiting factor for photometric measurements in these bands. At $350 \mu \mathrm{m}(860 \mathrm{GHz})$, while Peterson et al. found that the median sky opacity at South Pole and Chajnantor (Chile) to be similar (1.20 vs. 1.39, respectively), the fluctuations in the opacity level were found to be $\sim 3$ times lower at Pole during the best observing conditions, when the opacity is below unity - i.e. when observations at these wavelengths could be made.

For CMBR measurements, made in the microwave bands, the South Pole has proved to be a superb site, as attested by the wealth of science that has been achieved (see 4.2). For instance, at $220 \mathrm{GHz}$ the zenith opacity is just $\sim 0.03$ and the median brightness power fluctuations are an order of magnitude lower than at any established temperate sites (at $\sim 38 \mathrm{mK}^{2} \mathrm{rad}^{-5 / 3}$ in Rayleigh-Jeans temperature units; Bussmann, Holzapfel \& Kuo, 2005).

The Turbulent Surface Boundary Layer A strong temperature inversion exists above the ice during the most stable of conditions in winter, produced by radiative cooling from the ice surface in the absence of sun light. The temperature can rise by up to $\sim 20^{\circ} \mathrm{C}$ in heights of a few tens of metres. Virtually all the turbulence responsible for seeing at optical and infrared wavelengths, the result of micro-thermal temperature fluctuations causing refractive index changes, is confined to this boundary layer. Dealing with the boundary layer is one of the most challenging problems facing designers of optical and infrared telescopes in Antarctica (see $\$ 5.2$, but if its mitigating effects can be overcome then the Antarctic plateau offers sharper imaging, more precise photometry and superior astrometric precision than measurements made from any other place on Earth. Of course, such a statement applies to any ground-based telescope, but the narrow boundary layer above the Antarctic plateau suggests that this might be readily accomplished there, for instance by building a tower that extends above it.

The free air seeing in the visible, above the surface boundary layer, is typically a factor of two better than the $\sim 0.7^{\prime \prime}$ seeing above the best temperate sites. The proximity of the turbulent boundary layer to the telescope also results in reduced values for the scintillation noise, since the 'lever arm', from the turbulent atmospheric cells that de-focus the light to the telescope, is much reduced. Since scintillation provides the limiting factor to the error in a flux measurement, this implies that improved photometric precision is also possible. 
Two further quantities are also very important in determining site quality; the coherence time and the isoplanatic angle of the turbulent cells. Since the cells are located close to the surface over the Antarctic plateau, and pass across the telescope field of view relatively slowly, the above two parameters are larger than found at temperate sites, where the cells are at high altitude and move rapidly. Longer coherence times and larger isoplanatic angles feeds into improved performance for adaptive optics systems (e.g. larger bandwidth, fainter reference stars). For an interferometer, it also results in improved signal to noise and improvement in the achievable astrometric precision (e.g. Lloyd et al. 2002, Storey 2004).

Considerable effort has been put into quantifying the relevant parameters - the free air seeing $\left(\varepsilon_{0}\right)$, isoplanatic angle $\left(\theta_{0}\right)$, Fried parameter $\left(r_{0}\right)$, coherence time $\left(\tau_{0}\right)$, the boundary layer turbulence $\left(C_{N}^{2}\right)$, its height $(h)$ and outer scale length $\left(L_{0}\right)$ - at the South Pole, Dome $\mathrm{C}$ and, most recently, Dome A, so as to determine the quality of these sites for prospective telescopes. While this qualification of site properties remains incomplete, substantial information is now available, or can be inferred through modelling, so that a quantitative picture of observing conditions over the Antarctic plateau can be deduced. It is sufficient to allow a detailed comparison with conditions at mid-latitude sites. We discuss some of this work below.

\subsubsection{Comparison of Seeing Conditions at Plateau Sites}

South Pole At the South Pole a steady breeze blows most of the time (the katabatic wind falling from the summit of the plateau, Dome A). This causes substantial seeing at the ice surface level, of order $1.5^{\prime \prime}$ in the visual (Loewenstein et al. 1998). It is produced almost entirely in the lowest 200-300 $\mathrm{m}$ above the ice (Marks et al. 1996, 1999, Travouillon et al. 2003a). On the other hand, there is no jet stream to produce the high-altitude seeing that temperate sites experience. As first pointed out by Gillingham $(1989,1993)$, the free air seeing above the surface boundary layer is much smaller than the surface seeing at temperate sites, a concept termed by Gillingham "super-seeing".

The depth of the boundary layer at the South Pole, while shallow, is still too high to consider raising a telescope beyond it. On the summits of the plateau, however, the wind speed is much reduced, leading to a significantly thinner surface boundary layer (Marks 2002, 2005) than at the Pole.

Dome $C$ Two decades of measurements of wind speeds at Dome $\mathrm{C}$, made with an automated weather station (AWS), showed them to be exceptionally low, averaging $\sim 3 \mathrm{~m} \mathrm{~s}^{-1}$ at the ice surface, with maximum gusts reaching only $\sim 10 \mathrm{~ms}^{-1}$ (Aristidi et al. 2005b). Such data gave promising intimations of a stable atmosphere that would provide superb seeing. When such seeing measurements were first made (with a robotic observatory at Dome C (the AASTINO; Lawrence et al. 2004; see Fig. 10) using both an acoustic radar (SODAR), sampling the boundary layer, and a multi-aperture scintillation sensor (MASS), sampling the whole atmosphere), a median value was obtained for the visual seeing of just $0.27^{\prime \prime}$, above a boundary layer whose height was found to be only $\sim 30 \mathrm{~m}$ thick. Subsequent measurements from Dome C by Agabi et al. (2006), after the winter opening of the station, making use of direct measurements of the turbulence using microthermal sensors flown on balloons, combined with total seeing measurements using differential image motion monitors (DIMMs), obtained similar results within the errors (median seeing $0.36^{\prime \prime}$, boundary layer height $36 \mathrm{~m}$ ), so confirming that the turbulence is primarily confined to the boundary layer. These results indicated that the visual seeing at Dome $\mathrm{C}$, above this boundary layer, is typically about a half that found at the best temperate sites. For $\sim 10 \%$ of the time Lawrence et al. found 
the seeing, above the narrow surface boundary layer, to be $<0.1^{\prime \prime}$, the lowest values ever recorded. The determination of the isoplanatic angle and coherence time, as measured by this combination of instruments, was also in reasonable agreement (5.7" and $7.9 \mathrm{~ms}$ for Lawrence et al.; 4.7" and $8.6 \mathrm{~ms}$ for Agabi et al.; both above $30 \mathrm{~m}$ ), and indicate that Dome $\mathrm{C}$ is an excellent site for both wide-field, high spatial resolution imaging and for infrared interferometry. Furthermore, measurements of the scintillation noise (Kenyon et al. 2006) show it to be the lowest so far measured on the Earth, making it an excellent site for precision photometric measurements as well. As a caution to the reader, however, it should be remarked that the above results have been deduced from relatively limited measurements. It would be desirable to improve the statistics of these data sets to fully characterise the seeing conditions at Dome C.

The statistics of 3.5 years of DIMM measurements from Dome C, placed on towers at different heights within the surface boundary layer, have been summarised by Aristidi et al. 2009 (also incorporating results from Trinquet et al. 2008 on the vertical distribution of turbulence). The boundary layer height was found to be variable, as well as volatile on short timescales, but has a sharp boundary, whose median height was inferred to be between 23-27 $\mathrm{m}$ based on its statistical properties. When the boundary layer is depressed below the height of the DIMMs, excellent seeing from the free air is obtained (median value $0.36^{\prime \prime}$ ), as clearly seen in the bimodal distribution of the seeing shown in Fig. 11. There is also a clear summer-winter difference in the seeing distributions; while the bimodal separation in each plot indicates when the seeing monitor was either lying inside or above the boundary layer (and the clear separation indicating its sharp boundary). More turbulent energy resides within the boundary layer in winter, as indicated by the larger values of seeing measured then. The seeing data also provide an estimate of the median value of size of the outer scale for turbulence (Ziad et al. 2008), of $\sim 7 \mathrm{~m}$ in winter (measured $3.5 \mathrm{~m}$ above the ice). These values are 2-3 times lower than measured at Mauna Kea and Paranal observatories, and have implication for improved $\mathrm{AO}$ and interferometric performance by reducing fringe excursion errors (though the extension of these results to a telescope placed above the boundary layer is not clear).

A particularly interesting result (Travouillon 2004, Aristidi et al. 2005a) relates to the daytime seeing in summer. Late in the 'afternoon' each day, around 5 p.m. local time, the surface boundary layer often disappears, leading to a free-air seeing of $\sim 0.4^{\prime \prime}$ just $0.8 \mathrm{~m}$ above the ice (see Fig. 12). At this time of the day the temperature gradient above the ice disappears. The isothermal temperature profile then leads to a great reduction in the turbulent energy and hence excellent surface seeing. This has particular implications for measurements of the Sun, which of necessity must be made in daytime, providing a period of $\sim 2$ hours each day where excellent imaging quality is directly obtainable.

The paper by Gredel (2010) provides a concise and clear summary of the site testing results from Dome C, conducted under the auspices of the European Union ARENA consortium, including the results described above.

An interesting recent development is the application of numerical meteorological models to the calculation of turbulence and seeing conditions over the Antarctic plateau, with eventual application towards the forecasting of the seeing. Lascaux et al. $(2009,2010)$ have compared (low-resolution) general circulation models and (higher resolution) mesoscale models for the calculation of meteorological parameters, with the particular application being to the modelling of $C_{N}^{2}$ turbulence profiles obtained from 15 balloon flights from Dome $\mathrm{C}$ in the 2005 winter by Trinquet et al. (2008). Perhaps unsurprisingly, the higher resolution, computationally more expensive, models work best. Lascaux et al. find they can model the wind gradients and temperature profiles with height reasonably well, including their rapid 
changes over the extent of the surface boundary layer. The boundary layer thickness in these mesoscale models is, in general, currently calculated to be $\sim 50 \%$ higher than that measured. The turbulence profile $\left(C_{N}^{2}\right)$ within the boundary layer is reproduced well, but deviates from the data above the boundary layer. There is a reasonable correlation between observed and predicted seeing, although still considerable scatter for individual data points. The overall agreement between models and data shows that this is a promising area of study, as well as emphasising the need for a much more comprehensive set of measured turbulence profiles to compare the models with. The authors intend to extend their calculations to other sites over the Antarctic plateau, and so provide quantitative model comparisons for the behaviour of the seeing conditions at each site over the year.

Dome A Site testing at Dome A, the highest point on the Antarctic plateau, only began in 2008, making use of a series of instruments attached to the PLATO automated observatory (Yang et al. 2009, Lawrence et al. 2009). Of necessity, the information obtained so far is limited, but it does suggest some remarkable properties for the site. While direct seeing measurements have yet to be made, the boundary layer has been probed using a specially designed acoustic radar able to sample on the 1m-scale (SNODAR; see Bonner 2009, 2010 and Fig. 13, indicating that it is even thinner than at Dome C. Over the first part of the winter period of 2009 its median height was found to be $14 \mathrm{~m}$ and its $25 \%$ quartile value to be just $\sim 10 \mathrm{~m}$ thick.

Ridge A An analysis by Saunders et al. (2009) of three of the key parameters $\left(\varepsilon_{0}, \theta_{0} \& \tau_{0}\right)$, together with their 'coherence volume' $\left(\theta_{0}^{2} \tau_{0} / \varepsilon_{0}^{2}\right.$; Lloyd 2004) was undertaken to determine the efficacy of an interferometer placed at different sites over the plateau. The study ranks the South Pole as the best site of any station, followed by Dome A and then Dome C (an order of magnitude worse). However, Ridge A was found to be the best location of all. Ridge $\mathrm{A}$ is closer to the null point for origin of the katabatic winds than Dome A. It also has lower temperatures and less cloud cover.

While the estimates made in this study are, of necessity, preliminary and based on extrapolations of current data sets combined with satellite telemetry, they do highlight the need to obtain extensive site data over the plateau before choosing a site that may be the best for a particular application. There is no single best site on the Antarctic plateau for all astronomical applications.

\subsection{Other Site Factors}

Clear Skies While the South Pole experiences clouds about half the time, Dome C appears to be a much clearer site. A variety of measurements made from automated cameras and by direct observations indicate photometric skies occur an exceptional $\sim 75-90 \%$ of the time (e.g. Ashley et al. 2005; Mosser \& Aristidi, 2007, Moore et al. 2008a). Satellite measurements indicate that Dome A and F should have similar cloud characteristics to Dome C, being clear $\sim 75 \%$ of the time in winter (Saunders et al. 2009).

Direct measurements of the clear sky fraction at Dome C, as well as of the effective duty cycle, were also obtained in astronomical experiments during the $2007 \& 2008$ winters as part of the small-IRAIT (Strassmeier et al. 2008) and ASTEP projects (Crouzet et al. 2010), which we discuss below.

Small-IRAIT used an optical CCD camera mounted on a $25 \mathrm{~cm}$ telescope to follow photometric variations simultaneously of two bright variable stars, continuously over a 10 


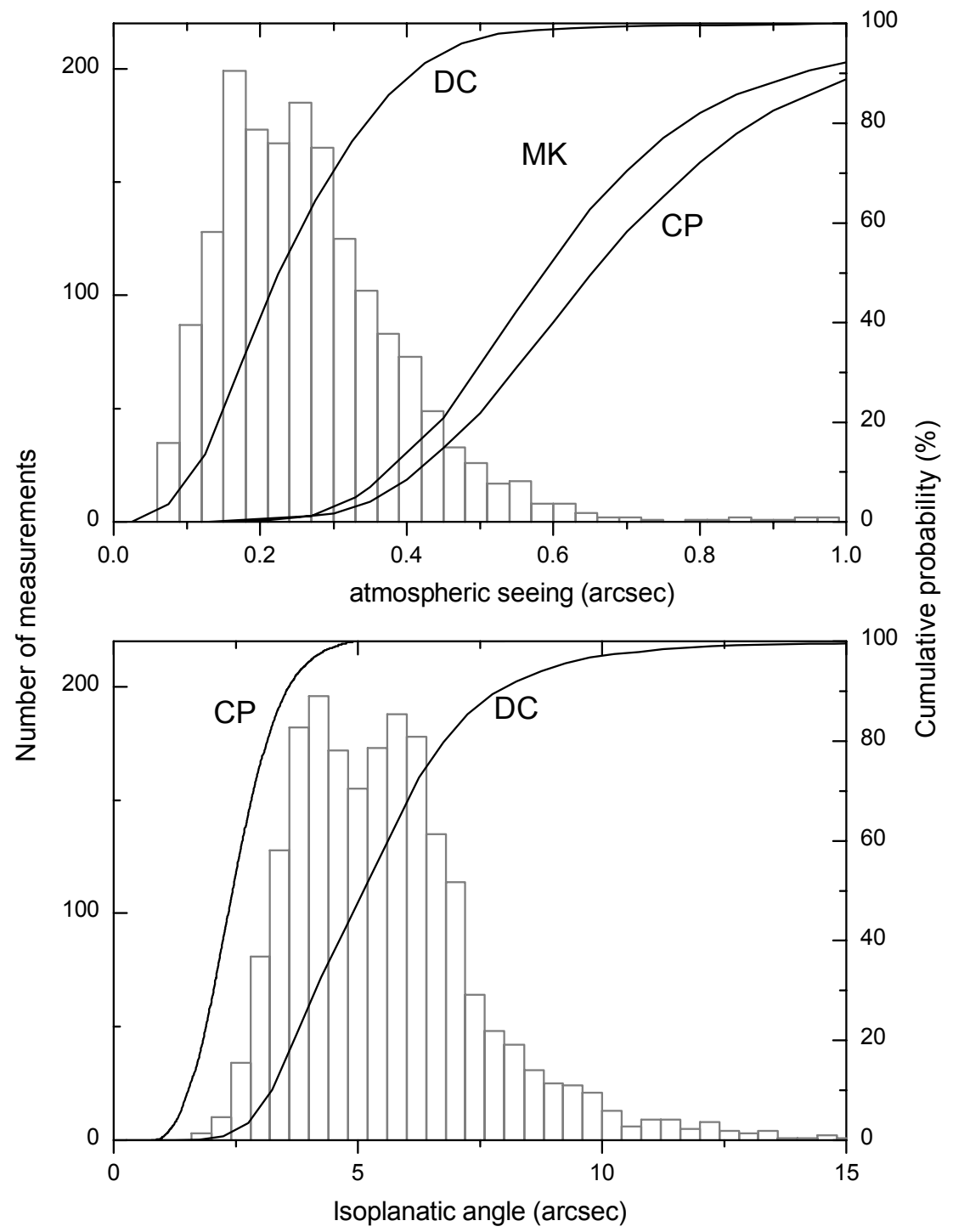

Fig. 10 Histograms and cumulative distributions for the atmospheric seeing (top plot) and isoplanatic angle (bottom plot) for Dome C, above $\sim 30 \mathrm{~m}$ (a typical boundary layer height), as measured by Lawrence et al. (2004) using a combination of MASS and SODAR instruments (see text in 2.3.1). The cumulative distributions also compare Dome C (DC) to Mauna Kea (MK) and Cerro Paranal (CP) (including the contribution from the surface boundary layer for these latter two sites). The superb seeing attainable close to the surface at Dome C (median value $0.27^{\prime \prime}$ in V-band) is evident, with the values even falling being below $0.1^{\prime \prime}$ at times. 

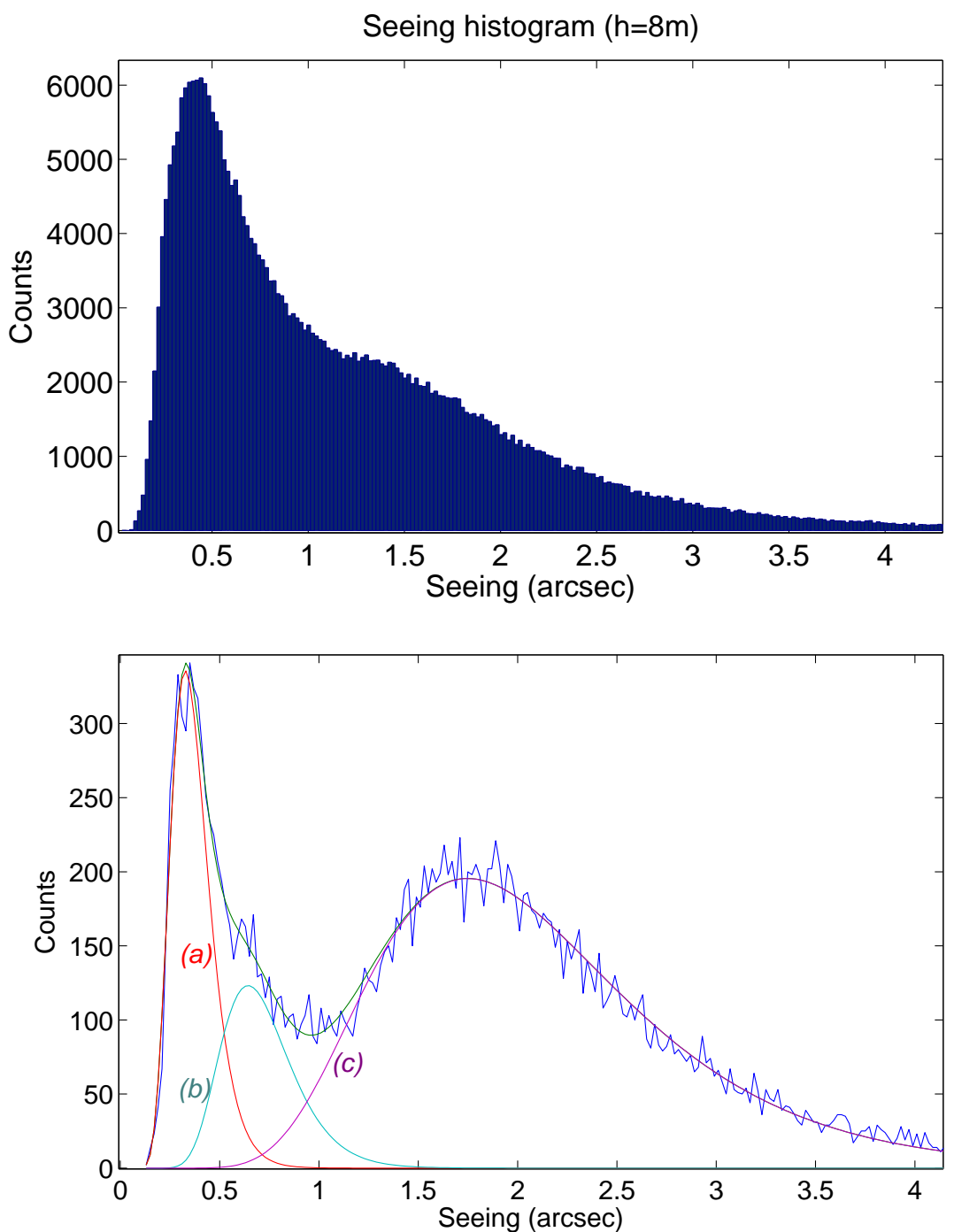

Fig. 11 Histograms of seeing measured with a DIMM at Dome $\mathrm{C}$ from Aristidi et al. (2009). The shaded histogram is the data from an instrument mounted $8 \mathrm{~m}$ above the ice, and includes the authors' full 3.5 year data set (summer \& winter). The bimodal distribution reflects periods of excellent seeing at this height, when the boundary layer drops below $8 \mathrm{~m}$, together with the more normal moderate seeing when the instrument lies within the boundary layer. The bottom curves show the data from the 2006 winter, with the three log-normal fits reflecting times when the instrument is above the boundary layer (a), within it (c) and a few intermediate cases (b). 

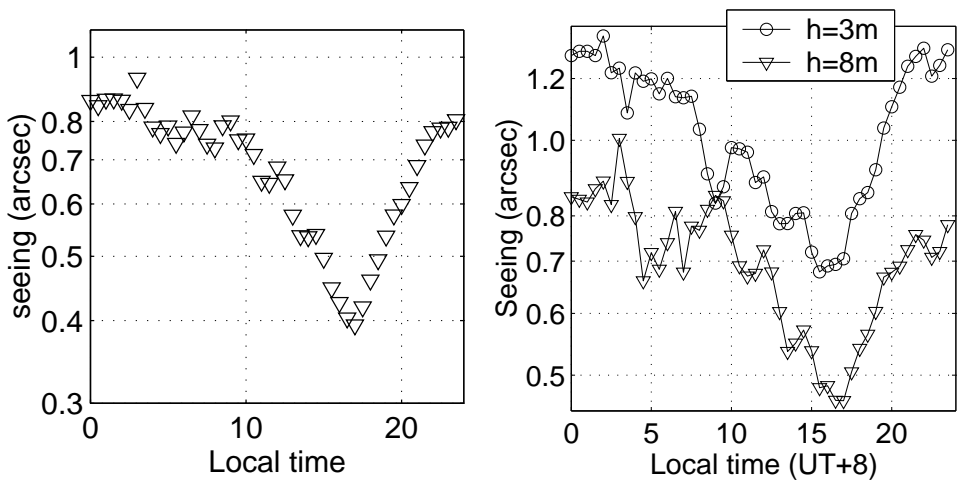

Fig. 12 Diurnal variation of the daytime seeing at Dome $C$, measured by Aristidi et al. (2005a). The plot to left shows data averaged over two summers (2003-04 \& 2004-05) measured from a DIMM placed $8 \mathrm{~m}$ above the ice. The plot to right compares data from two DIMMs, one at $8 \mathrm{~m}$ and the other at $3 \mathrm{~m}$ above the ice, from the 2004-05 summer. Note especially the behaviour each afternoon, around 5 p.m. local time, with the boundary layer often disappearing. This is caused by the temperature gradient above the ice disappearing for a few hours then. This results in excellent "on-ice" seeing of around 0.4 ", with implications for obtaining high image quality for solar telescopes.

day period in July 2007. The weather was stable and only $\sim 6$ hours were lost over this time period, providing a duty cycle of $98 \%$. Furthermore, a 3 mmag photometric precision in V-band (and 4.2 mmag in R-band) was obtained for a 2.4 hour subset of the data, 3-4 times better than has been achieved with an equivalent telescope in Arizona, attesting to the improved photometric precision resulting from reduced scintillation noise.

ASTEP used a CCD camera on a fixed $10 \mathrm{~cm}$ refractor pointed towards the celestial South Pole. Excellent sky conditions were recorded $\sim 60 \%$ of the time during the 4 months of operation (June - September, 2008). A duty cycle of 50\% for photometric measurements in the optical was obtained (taking into account cloud, moonlight, scattered sunlight etc.), a higher value than has been obtained at temperate sites.

Sky brightness and transparency measurements have also been made at Dome A in the winter of 2008 by the CSTAR (the Chinese Small Telescope ARray) instrument (Zou et al. 2010), controlled from the PLATO laboratory. This comprises four fixed $14.5 \mathrm{~cm}$ telescopes, pointed at the south celestial pole, each with a $4.5^{\circ}$ field and a different optical filter. The primary purpose of this experiment was to search for variability in stellar flux, for which a catalogue of 10,000 stars and 30,000 images has been produced (Zhou et al. 2010). Clear skies were found in $74 \%$ of the images.

Aircraft Contrails Aircraft contrails add to high-altitude turbulence and increase optical sky brightness via scattering of moonlight. They are an increasing problem at temperate sites as they are often overflown by air traffic, whose frequency continues to increase. Contrails are, however, non-existent over the Antarctic plateau during the winter months.

Low Aerosols and Pollution Antarctica has the cleanest air on the Earth, in particular in the amount of aerosols in the atmosphere (there is no dust). For instance, as discussed in Kenyon \& Storey (2006), aerosol levels are $\sim 50$ times lower than found at the atmospheric observatory on Mauna Loa, Hawaii (Bodhaine, 1995). This results in reduced emissivity at infrared wavelengths (and so lower sky emission) and reduced levels of scattering at optical 

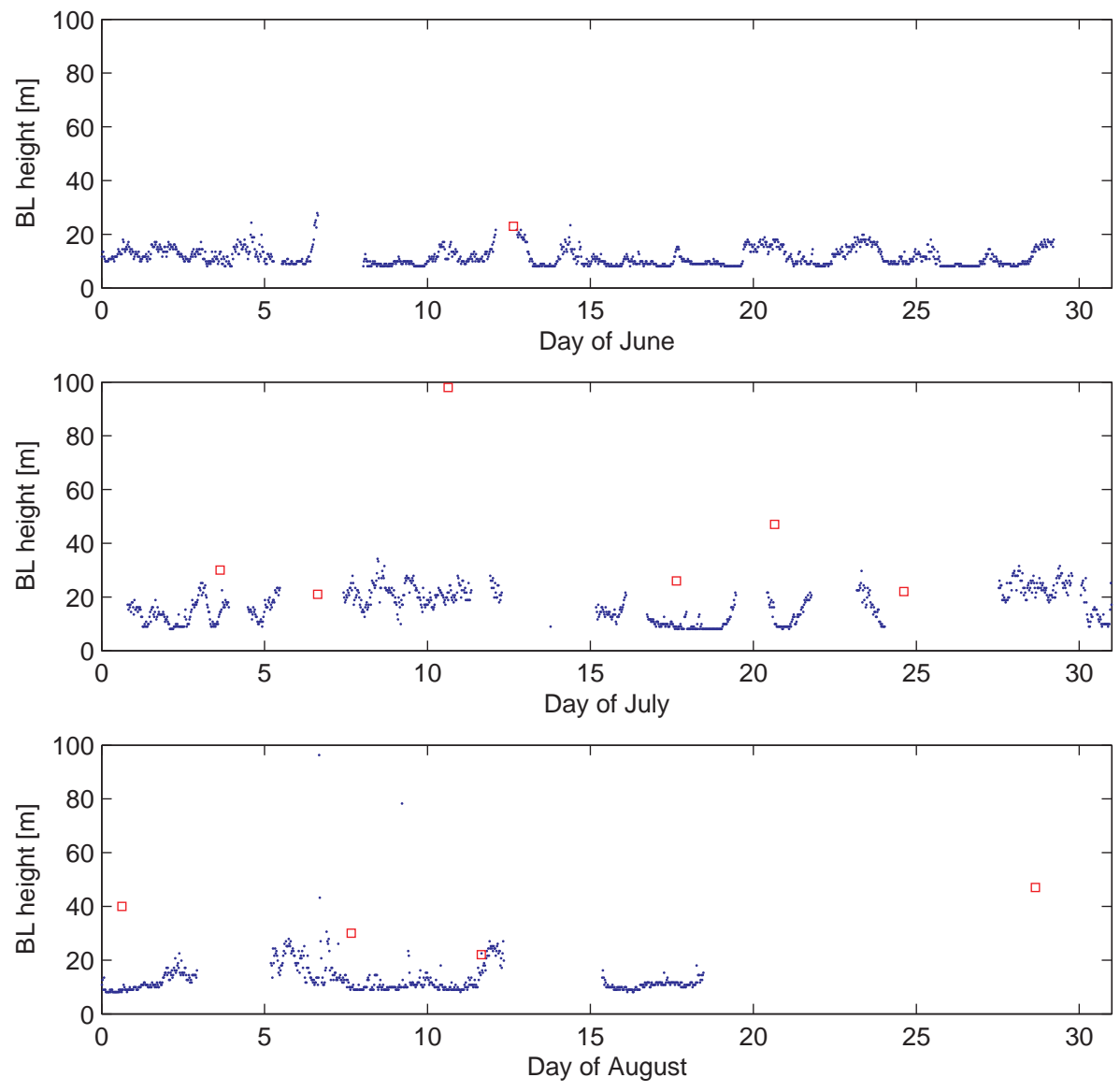

Fig. 13 Height of the boundary layer at Dome A from June to August 2009, as measured using the SNODAR instrument (an acoustic radar; Bonner et al. 2010). Each dot represents the average of 30 minutes of data. Squares show measurements made at Dome C on the same day of the year in 2005 by Trinquet et al. (2008) from balloon measurements. The SNODAR provided a vertical resolution of $0.9 \mathrm{~m}$ and sampled the turbulence in the atmosphere every $10 \mathrm{~s}$ to a height of $180 \mathrm{~m}$ above the ice, to determine the temperature structure constant, $C_{T}^{2}$. The boundary layer height was defined to be the height at which $C_{T}^{2}$ reduces to $1 \%$ of its initial value. The $25 \%, 50 \%$ and $75 \%$ quartiles determined for it by Bonner et al. (2010), over the Feb-Aug period of their whole data set, are $9.7 \mathrm{~m}, 13.9 \mathrm{~m}$ and $19.7 \mathrm{~m}$, respectively.

wavelengths. It is, of course, important to avoid local degradation of these conditions at an observatory, for instance as might be caused by emissions from diesel generators. This might be achieved by the use of clean energy sources (see also $\S 5.2$ ).

There is also minimal light pollution to consider during the winter months, as there are no settlements nearby other than the scientific station the observatory is based at, and this will remain the case for the foreseeable future.

Seismically Quiet Antarctica is the quietest continent seismically, quite different to the geologically active regions where temperate observatories are often located (e.g. in Hawaii or Chile). While not normally a critical part of the assessment in designing telescopes, consid- 
eration of the effects of seismic disturbances have implications for the design of the largest telescopes, such as the ELTs, or interferometers where path lengths must be maintained to sub-micron precision. While the Antarctic ice sheet itself is moving, at about $10 \mathrm{~m}$ per year, this is a bulk motion that is straightforward to measure and to compensate for.

\subsection{Disadvantages of Antarctica for Astronomy}

There are, of course, some disadvantages relating to the site conditions on the Antarctica plateau. The cold and high altitude pose difficulties for humans, but have relatively simple engineering solutions for the operation of facilities. Other facets of the sites are less easily overcome. Here we summarise the principal issues, and discuss their effects further in $\$ 5$.

Humidity and Super-Saturation While the extreme cold means that the absolute humidity level of the air is low (up to three orders of magnitude lower than at temperate sites), the relative humidity in Antarctica is always high. Calculations assuming 100\% humidity in fact provide reasonable approximations for the atmospheric transmission, without needing a detailed knowledge of its actual level and distribution through the atmosphere. Close to the ice surface, within the boundary layer, the air can in fact be super-saturated. This has the adverse consequence of causing ice growth on any exposed surfaces, unless they are heated slightly above the ambient temperature, flushed with dry air, or placed within an enclosure in a thermally controlled environment (see also $\S 5.2$ ).

Sky Coverage The further a telescope is sited from the Equator the less sky it has access to. In the extreme case of the Poles only half the celestial sphere is visible, though of course it is visible the whole time. An even smaller portion of sky is accessible above a telescope elevation of $30^{\circ}$, the usual elevation limit considered for most observations. At Dome $\mathrm{C}$ (75S) only $37 \%$ of the sky can be seen above $30^{\circ}$ compared with, for instance, $81 \%$ from Mauna Kea (see Kenyon \& Storey, 2006). Those objects that can be seen, however, may be observed for longer, and suffer less diurnal change in elevation than they do when seen from temperate sites, providing gains in precision for long time-series measurements.

Twilight The Sun also spends long periods of time just below the horizon at high latitude sites, meaning that the fraction of time that is "astronomically dark" (i.e. when the Sun is more than $18^{\circ}$ below the horizon) is smaller than at temperate latitudes. This is only relevant at optical wavelengths, as at infrared wavelengths the sky is bright (even in Antarctica). However, the reduced aerosol content reduces the scattering, so that the sky is dark in Antarctica with the Sun closer to the horizon than is the case at temperate sites. In the short infrared wavebands $(1.6 \& 2.4 \mu \mathrm{m})$, Phillips et al. (1999) found the effect of scattered sunlight disappeared when the Sun is more than $10^{\circ}$ below the horizon at the South Pole. Moore et al. (2008a) and Crouzet et al. (2010) found that at Dome C the sky is optically dark when the Sun is more than $13^{\circ}$ below the horizon (as did Zou et al. 2010 at Dome A). Combining this limit with the fraction of clear skies and with the phase of the Moon, Kenyon \& Storey (2006) find that the number of hours of optically dark time available at Dome C per year is at least as many as at Mauna Kea. 
Auroral Activity and Optical Sky Brightness Auroral activity in Antarctica is frequent, but its intensity varies over the continent, dependent on whether the auroral circle is visible. The South Pole and Dome F lie nearly under the auroral circle, so suffer frequently, whereas Domes A, B \& C lie just $6^{\circ}$ from the geomagnetic South Pole, so that aurorae there generally lie below the horizon. Auroral emission is concentrated into a few spectral lines, particularly atomic oxygen and bands from molecular nitrogen and oxygen. Aurorae will therefore only impact on a small subset of observations that might be undertaken from Antarctica. Saunders et al. (2009) re-analyse the work of Dempsey \& Storey (2006) 1 on auroral emission, extending it over the principal sites of interest on the plateau. They find the auroral sky contribution $\left(\sim 23\right.$ mags $\operatorname{arcsec}^{-2}$ at B \& V) at the best plateau sites (Domes A, B \& C), will make the sky there about a factor 2 brighter than at the best temperate sites at $\mathrm{B}$ band (and 20-30\% brighter at V). The South Pole and Dome F, on the other hand, are another 1-2 magnitudes worse. By way of a direct comparison of their impact on an experiment, ASTEP, operating in the optical over the 2008 winter at Dome C (Crouzet et al. 2010), found negligible influence from aurorae on their data set. The CSTAR experiment at Dome A (Zou et al. 2010) determined the typical sky brightness in the Gunn $i$ band, in dark sky conditions in mid-winter, to be 20.2-20.5 mags $\operatorname{arcsec}^{-2}$. Converting this to the Mould I band (subtracting 0.75 magnitudes; see Windhorst et al. 1991), this is comparable to darkest skies measured at dark temperate sites such as Siding Spring Observatory in Australia (19.3 mags $\operatorname{arcsec}^{-2}$ ) and CTIO in Chile (19.9 mags $\operatorname{arcsec}^{-2}$ ).

\section{A Brief History of Antarctic Astronomy}

Captain Cook, and the crew of the ships Resolution and Adventure, were the first people to cross the Antarctic Circle in 1772, carrying with them a collection of telescopes, quadrants and chronometers, together with two astronomers, William Wales and William Bayly (Wales $\&$ Bayly 1777). However the prime purpose of these instruments was for navigation rather than astronomy. Douglas Mawson's Australasian Antarctic Expedition of 1911-1914 can be credited with first advancing the science of astronomy in Antarctica, through the discovery of the Adelie Land Meteorite. This was found on December 5, 1912, during one of the many sledging expeditions undertaken to chart the coastal fringes around Commonwealth Bay in Adelie Land (Bayly \& Stillwell, 1923). It was not until 1969, however, before Japanese scientists found a number of meteorites of different types in close proximity and realised the favourable conditions that Antarctica provides for their collection - the meteorites are transported from where they fall to ablation zones in blue ice fields, where they can simply be picked up off the snow (see Nagata 1975). Over 30,000 meteorites have now been found in Antarctica, more than from the rest of the world put together. Meteorite research is currently of particular interest due to the discovery of several Martian meteorites in Antarctica, and the possibility they may contain signatures of past biotic structures from that planet (e.g. McKay et al. 1996).

Admiral Peary, who had led the first successful expedition to the North Pole in 1909, wrote to the Director of Yerkes Observatory, EB Frost, in 1912 2 suggesting that Antarctica might provide a suitable place to consider continuous observations of astronomical sources

\footnotetext{
1 This paper contains an error in the position used for the Geomagnetic Pole, implying that Dome C is closer to the Geomagnetic Pole than Dome A. The error is rectified in Saunders et al. (2009).

2 Exchange of letters between RE Peary \& EB Frost, held at Yerkes Observatory, Wisconsin, USA and reproduced in the newsletter of the Center for Astrophysical Research in Antarctica (CARA), Yerkes Observatory (\#5, January 1994).
} 
on account of the long winter night. Frost replied largely in the negative, and the subject was not pursued further. The first measurements to be made for astronomical research were to study cosmic rays. Cosmic-ray detectors were installed at the Australian base of Mawson in 1955 (Parsons 1957) and at the US base of McMurdo during the International Geophysical Year of 1957 (Pomerantz, Agarwal \& Pontis 1958), the year when Antarctic science began in earnest. It was not until 1979 that the first optical research programs were conducted in Antarctica, when 120 hours of continuous observation of the Sun was made using an $8 \mathrm{~cm}$ diameter heliostat at the South Pole (Grec et al. 1980). A $45 \mathrm{~cm}$ sub-mm telescope was then re-deployed from the Canada-France-Hawaii $3.6 \mathrm{~m}$ Telescope and used for site testing at the Pole in 1984 (Pomerantz 1985), and by the end of that decade several experiments had been trialled for measurement of CMBR anisotropies. A $2^{\prime \prime}$ periscope-style optical telescope, SPOT (the South Pole Optical Telescope), was also operated at the Pole from 1984-1988, managing to obtain a continuous light curve of 1 weeks duration of the Wolf-Rayet star $\gamma^{2}$ Velorum (Taylor 1990).

Antarctic astronomy began to flourish in earnest from 1991, following the formation in the USA of CARA, the 'Center for Astrophysical Research in Antarctica' at the South Pole, with Doyal Harper, Director of Yerkes Observatory (a part of the University of Chicago), as its first Director. CARA instigated research programs into CMBR anisotropy, sub-millimetre and infrared astronomy, as well as initiating a site testing program. Australia, through the University of New South Wales, joined this last program in 1994, and began designing experiments to allow uninhabited high plateau sites to be evaluated (Storey et al. 1996).

Construction of the Martin A Pomerantz Observatory (MAPO) began at the Pole with CARA, and continues to grow today. A series of telescopes have been deployed for CMBR measurements: the $0.75 \mathrm{~m}$ Python (Coble et al. 1999), the $2.1 \mathrm{~m}$ Viper (Peterson et al. 2000), the 13-element DASI interferometer (Leitch et al. 2002), the $25 \mathrm{~cm}$ BICEP (Takahashi et al. 2010) and currently the $10 \mathrm{~m}$ South Pole Telescope (SPT; Carlstrom et al. 2010). CMBR experiments were also flown on high-altitude balloons, launched from the Long-Duration Balloon Facility at the coastal station of McMurdo (e.g. BOOMERanG, de Bernadis et al. 2000; BLAST, Pascale et al. 2008). For the sub-millimetre, the $1.7 \mathrm{~m}$ off-axis AST/RO telescope was constructed at the South Pole, and used with a series of increasingly more sophisticated instruments (Stark et al. 2001). In the near-infrared the $0.6 \mathrm{~m}$ SPIREX telescope was deployed, equipped with cameras from 1-5 $\mu \mathrm{m}$ (Hereld et al. 1990, Fowler et al. 1998).

The CMBR facilities, in particular, have produced a wealth of discoveries (see also 4 4.2, including the first conclusive measurements that the geometry of the Universe is flat (de Bernardis et al. 2000), of the polarization of the E-modes of the CMBR (Kovac et al. 2002) and of the thermal-SZ effect in galaxy clusters (Staniszewski et al. 2009).

Concurrently with these developments, a number of increasingly sophisticated highenergy experiments were being developed at the Pole, seeking cosmic sources of gamma rays, cosmic rays and neutrinos (see also 4.6). These started with GASP (Morse \& Gaidos, 1990), a gamma-ray telescope at the South Pole, developed through the SPASE cosmic ray air shower array (Smith et al. 1989) and then led to the AMANDA neutrino detector (Andrés et al. 2000). This was a fore-runner to IceCube, which makes use of a cubic kilometre of pure ice to search for sources of high-energy neutrino emission (Ahrens et al. 2004). At a cost of over US\$300M, IceCube is the single most expensive scientific experiment ever to be conducted in Antarctica. The USA has significantly upgraded the infrastructure at the South Pole to cope with the increasingly sophisticated astronomical experiments being deployed there.

The latest chapter in the Antarctic astronomy is taking place on the summits of the high plateau, Domes A, C \& F. It began at Concordia station (France \& Italy) at Dome C, with 
the first summer time measurements made in 1996-97 by Valenziano \& dall'Oglio (1999), of precipitable water vapour in the atmosphere. The first winter-time operations at the station took place in 2005 (Fossat 2005), though the first winter astronomical measurements were carried out in 2003 using the AASTINO automated observatory (Storey et al. 2003, Lawrence, Ashley \& Storey 2005). Construction of Kunlun station at Dome A began in 2009 (China), but astronomical measurements began there in the 2008 winter season using the PLATO automated observatory (Yang et al. 2009). At Dome F site testing has been initiated, with summer time measurements of the millimetre sky transparency and turbulence in the lowest kilometre of the atmosphere obtained (Ichikawa, 2010). However, analysis of an ice core drilled in 2001 at Dome F for paleoclimate research has found spikes of enhanced nitrate ion concentration. These may be attributable to atmospheric ionization caused by gamma rays produced by two historical supernovae (Motizuki et al. 2010). There is also evidence for the 11-year solar cycle in the same data. If these findings are substantiated, there is the exciting prospect of extending this technique further back in time to search for pre-historic supernovae in the ice core record.

A full account of the historical development of astronomy in Antarctica to 2004 can be found in Indermuehle, Burton \& Maddison (2005).

\section{Astronomical Results from Antarctica}

Despite the relative youth of the field, and the small size of the science community working in Antarctica, a diverse and extensive range of astronomy has been conducted from the continent. Here we briefly overview some of the science results that have been obtained.

\subsection{Helioseismology}

The first observations for astronomical research at the South Pole took place in 1979 in a program to study the interior of the Sun. A sodium resonance cell was attached to an $8 \mathrm{~cm}$ telescope. An unbroken run of measurements over an unprecedented continuous 5 days length was obtained, of the $5,896 \AA \mathrm{Na}$ D1 line over the full disk of the Sun, and reported in a paper to Nature (Grec, Fossat \& Pomerantz, 1980). A clear signal of pulsation was seen, dominated by the 5 minute global solar oscillation. An array detector was added in the 198182 summer, allowing features to be resolved as small as $10^{\prime \prime}$ on the solar disk. A full analysis (Grec, Fossat \& Pomerantz, 1983; see Fig. 14) found $~ 80$ harmonics of solar eigenmodes, with periods ranging from 3 to 8 minutes. The latitude-dependent measurements provided evidence that the structure of the convection zone in the Sun is different near the equator to that at higher latitudes. These pioneering measurements have been an important step leading to our detailed knowledge today of the temperature, composition and motions in the Sun's interior, helping develop the techniques later used in spacecraft (SOHO - the Solar and Heliospheric Observatory; Gabriel et al. 1995) and with the GONG telescope network (Global Oscillation Network Group; Kennedy et al. 1994).

An $80 \mathrm{~cm}$ solar telescope was also flown on two long duration balloon flights, launched from McMurdo in 1996 \& 2000 - the Flare Genesis Experiment (Bernasconi et al. 1999). Imaging of solar flares and filament eruptions was carried out, although the image quality obtained, of $0.5^{\prime \prime}$, was poorer than the diffraction-limited $0.1^{\prime \prime}$ aimed for. The balloon gondola and telescope is to be re-used for the STO THz astronomy flight in 2011 (see 6.1 ). 


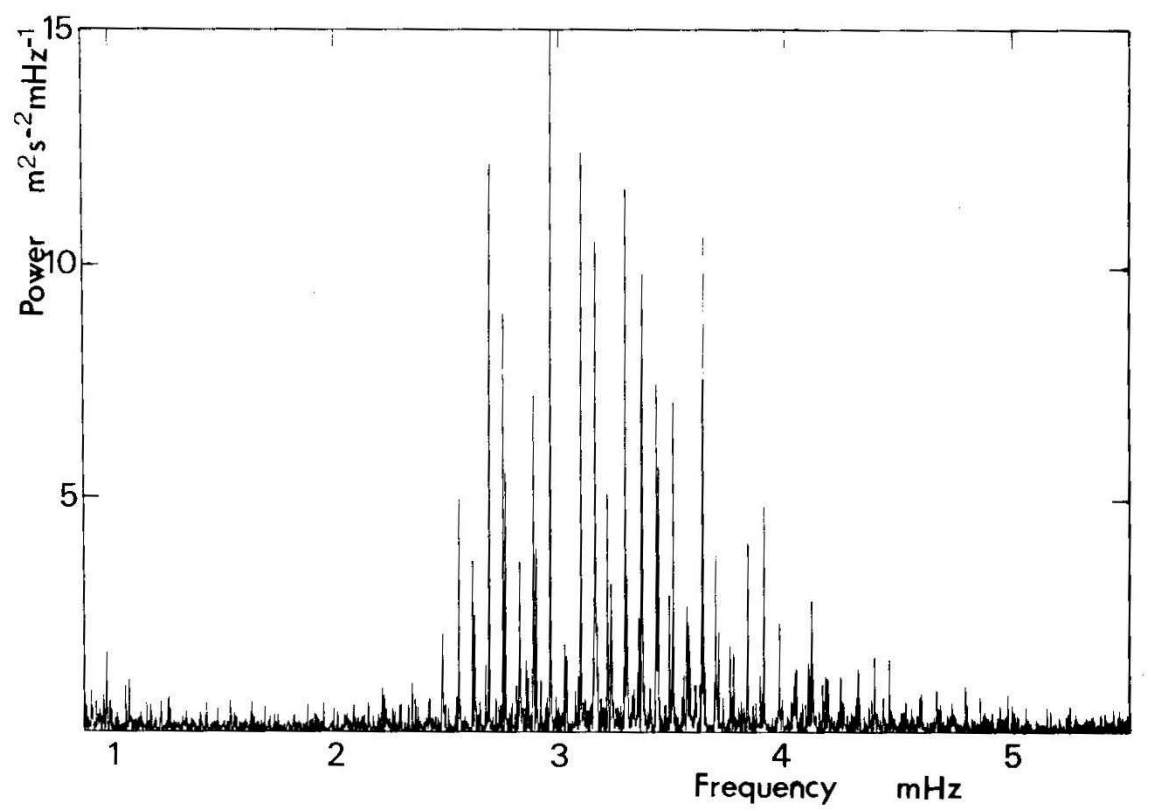

Fig. 14 A power spectrum obtained from 5 days of continuous observations of motions on the surface of the Sun using a sodium resonance cell, as made at the South Pole by Grec, Fossat \& Pomerantz (1980). Over 80 harmonics are seen, with periods ranging from 3 to 8 minutes (i.e. the 5 minute solar oscillation). The many lines separated by $68 \mu \mathrm{Hz}$ represent the global $p$-modes of oscillation of the Sun, with the smallest reliable features, seen around $2.4 \mathrm{mHz}$, corresponding to oscillations with speeds of $<10 \mathrm{~cm} \mathrm{~s}^{-1}$ and displacements $<5 \mathrm{~m}$ on the Sun's surface.

Interest in using Antarctica as a platform for Solar studies has recently been revived by the discovery of regular periods of excellent daytime seeing at Dome $\mathrm{C}$, as well as the high clear sky fractions at that site (see $\$ 2.3$, e.g. Damé et al. 2010). The scientific focus for future Antarctic solar observations is now on high spatial resolution imaging of the coronachromosphere interface, including direct measurements of magnetic fields.

\subsection{Cosmic Microwave Background Anisotropies}

Measurements of CMBR anisotropies have provided the most prominent science to emerge from astronomy in Antarctica to date. This has been facilitated by the extreme stability of the atmospheric microwave emission over the Antarctic plateau, making it the pre-eminent earth-based site for CMBR measurements. There were a series of experiments, starting in the mid-80's (see Indermuehle et al. 2005 for the early history), but it was not until the $1.4 \mathrm{~m}$ "White Dish" experiment (Tucker et al. 1993) that new science results in the field emerged, placing tighter constraints on CMBR anisotropies at high angular scales than had been determined by the COBE satellite.

At McMurdo, the BOOMERanG experiment (Balloon Observations Of Millimetre Extragalactic Radiation ANd Geomagnetics) carried a $1.2 \mathrm{~m}$ microwave telescope to an altitude of $38 \mathrm{~km}$ on a long-duration balloon flight in 1998. The experiment combined broad frequency coverage with the high sensitivity obtained from the 10 days duration of the flight 

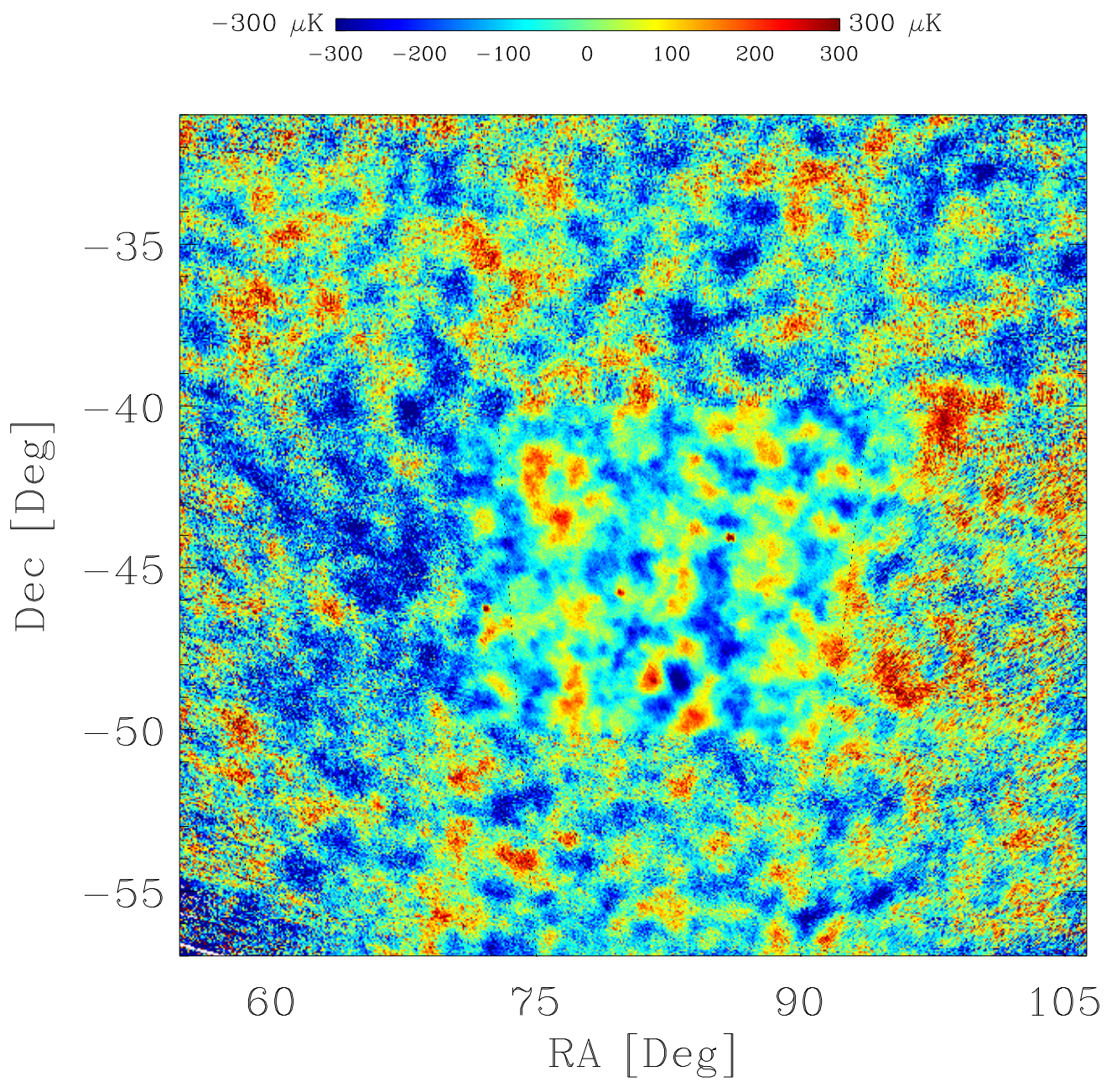

Fig. 15 Map of the CMBR fluctuations measured at $145 \mathrm{GHz}$ by the BOOMERanG long duration balloon experiment flown from McMurdo (de Bernardis et al. 2000; this image as reported with further data from Masi et al. 2006). The colour scale marks fluctuations between $\pm 300 \mu \mathrm{K}$ about the CMBR mean temperature of $2.725 \mathrm{~K}$. The pixel scale of the map is $3.4^{\prime}$. This data provided the most convincing evidence then available for the Euclidean nature of the Universe; i.e. that the geometry is flat.

through exceptionally clear atmospheric windows. The data provided the best evidence at the time that the geometry of the Universe was indeed flat (i.e. Euclidian; de Bernardis et al. 2000 - see Fig. (15). A second flight in 2003 then obtained polarization maps (Masi et al. 2003) of the E-mode of the CMBR anisotropy fluctuations.

DASI (Degree Angular Scale Interferometer) was a 13-element interferometric array built at the South Pole designed to extend the angular coverage of the CMBR to values in 


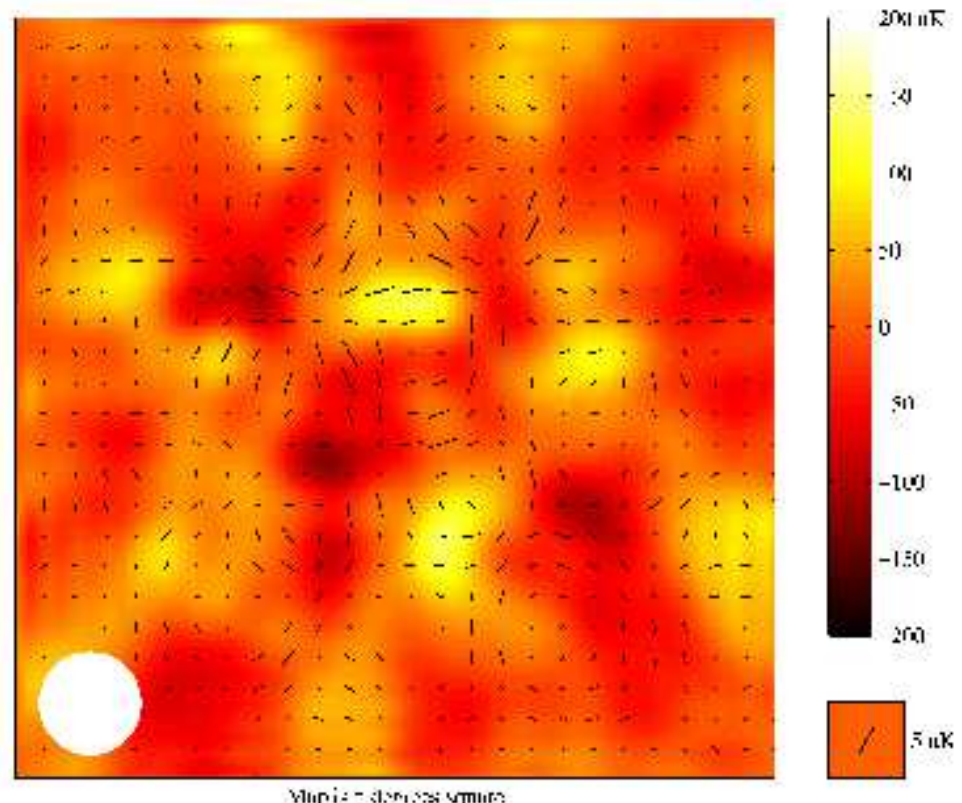

Fig. 16 The first measurement of polarization made of the CMBR, obtained by the DASI experiment at the South Pole (Kovac et al. 2002). The figure shows the E-mode polarization vectors overlaid on an image showing the CMBR fluctuations over the $5^{\circ}$ field of view. The beam size is shown by the white circle.

the range $140<l<900$. It made the first detection of polarization in the CMBR (Kovac et al. 2002 - see Fig. 16, obtaining a $5 \sigma$ detection of the "E-mode" of the CMBR polarization. The QUaD experiment, which combines a 31-element bolometer array with DASI, has now extended this imaging of the E-mode polarization to over $\sim 800$ square degrees, at an angular resolution of 5 arcminutes (Castro et al. 2009; Culverhouse et al. 2010).

With the 16-pixel ACBAR array receiver on the $2.1 \mathrm{~m}$ Viper telescope at the South Pole, Kuo et al. (2004) obtained the highest signal-to-noise measurements then made of CMBR anisotropy at high angular scales $(l=100-3,000)$. Combined with data from other CMBR experiments (in particular the WMAP satellite), this led to the best estimates then available of important cosmological parameters such as the Hubble constant, the age of the Universe, and the contributions of matter \& dark energy to the overall composition of the Universe (see Spergel et al. 2003).

BICEP is a $25 \mathrm{~cm}$ telescope currently operating at the South Pole. Using a 49-element bolometer array, it is designed to probe the polarization of the CMBR on degree angular scales, to search for the signature of "B-mode" gravitational waves. These are produced during the epoch of inflation. After 2 years of operation BICEP has probed for B-modes an order of magnitude deeper than any previous experiment (though they are yet to be detected; 


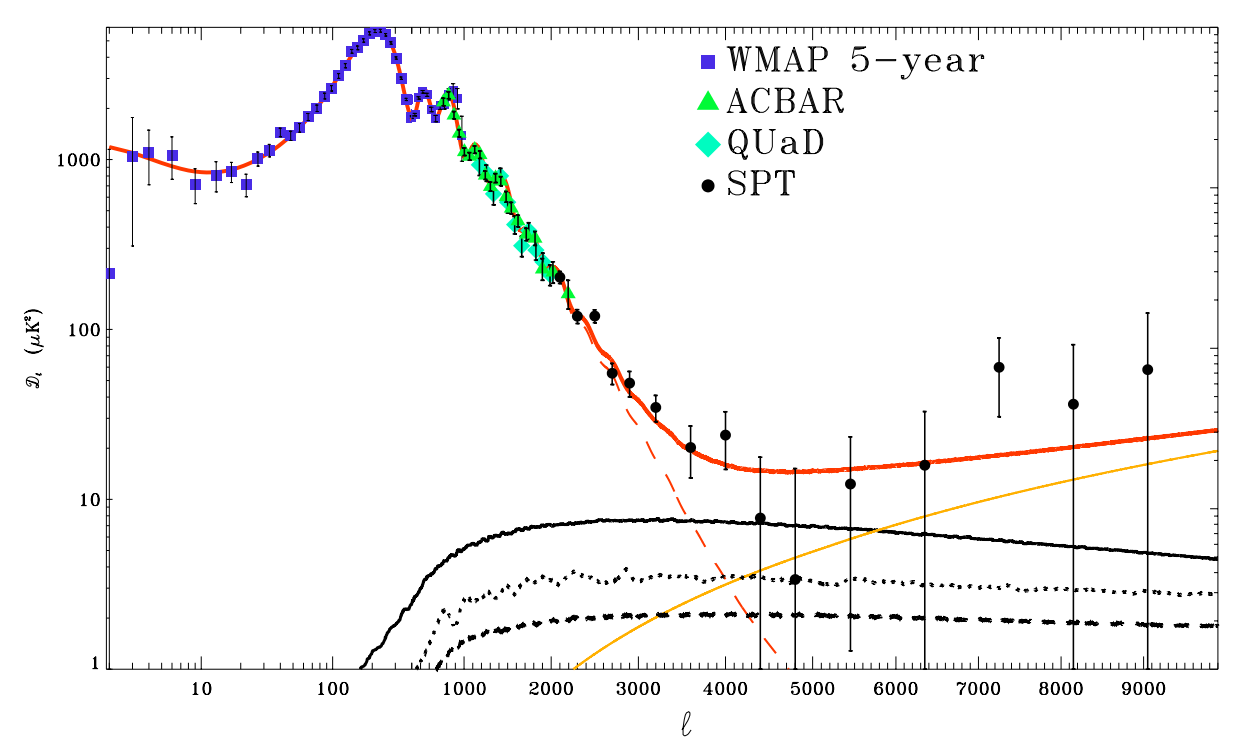

Fig. 17 Angular power spectrum of the temperature anisotropies in the CMBR, measured out to an angular scale of $l=9,500$, as shown in Lueker et al. (2010). The power spectrum incorporates results from the WMAP satellite at small values of $l$, from the ACBAR \& QUaD experiments at the South Pole on intermediate scales, and from the SPT results on the largest scales. For small values of $l$ the data is consistent with predictions from $\Lambda \mathrm{CDM}$ cosmology (red-dashed line), but at high $l$ there is a clear deviation from this model. A combination of contributions from the thermal SZ-effect (solid black line), the kinematic SZ-effect (dashed black line; note the dotted black line also represent the kSZ-effect, from 'patchy' re-ionization models) and from point sources (orange line) gives the red curve that provides the best fit to the data. This last component is presumed to arise from dusty, star-forming galaxies.

see Chiang et al. 2010), as well as making the first detection of the first peak in the angular power spectrum of the E-mode polarization.

The largest telescope to be so far operated in Antarctica is the $10 \mathrm{~m}$ off-axis South Pole Telescope (SPT), employing a bolometer camera with nearly 1,000 pixels (Carlstrom et al. 2010) and working at three frequencies $(90,150 \& 220 \mathrm{GHz})$. Its key projects are to detect clusters of galaxies via the Sunyaev-Zeldovich (SZ) effect and to measure the high- $l$ angular power spectrum of the CMBR, over a $\sim 1,000$ square degree area of sky. The first results from SPT have been published. Images of the thermal-SZ effect in several clusters are shown in Staniszewski et al. (2009) and Plagge et al. (2010). The power spectrum of the temperature anisotropies, extended out to an angular scale of $l=9,500$ is shown in Fig. 17 from Lueker et al. (2010). This power spectrum incorporates the results from the WMAP satellite at small values of $l$, from the ACBAR \& QUaD experiments at the South Pole on intermediate scales, as well as the SPT results on the largest scales. While for small values of $l$ the spectrum is consistent with that predicted by $\Lambda \mathrm{CDM}$ cosmology, at high $l$ there is clear evidence for a contribution from point sources, presumed to be dusty, star-forming galaxies, now seen for the first time in the angular power spectrum. 


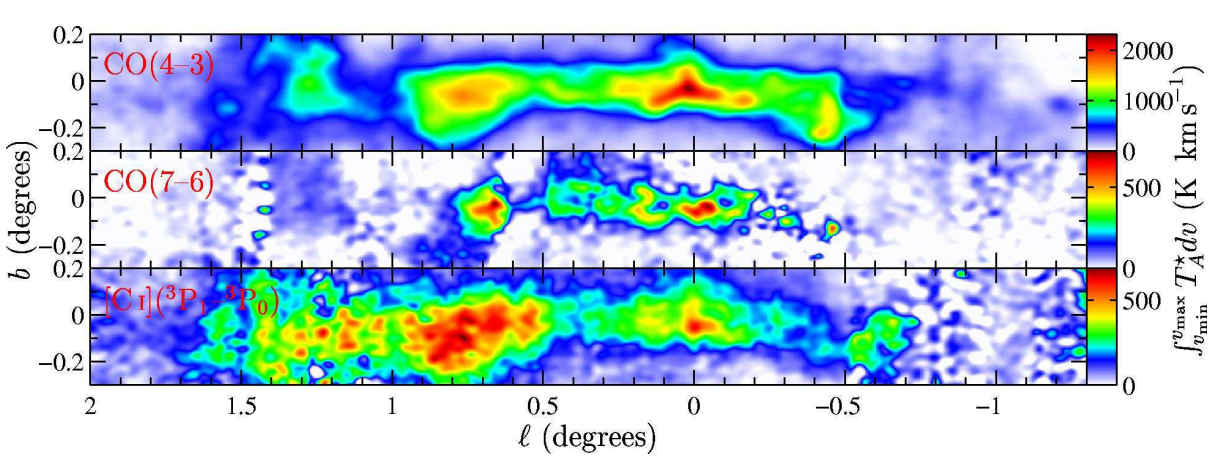

Fig. 18 Maps obtained in the sub-millimetre by the $1.7 \mathrm{~m} \mathrm{AST/RO} \mathrm{telescope} \mathrm{at} \mathrm{the} \mathrm{South} \mathrm{Pole} \mathrm{showing} \mathrm{the}$ 'Central Molecular Zone' of our Galaxy, a $3^{\circ} \times 0.5^{\circ}$ region across the galactic centre containing $\sim 10 \%$ of the Galaxy's molecular gas (Martin et al. 2004). The three maps show the distribution of CO J=4-3, CO J=7-6 and $[\mathrm{CI}]{ }^{3} \mathrm{P}_{1}-{ }^{3} \mathrm{P}_{0}$ line emission, at $372,651 \& 370 \mu \mathrm{m}$ respectively. They have $\sim 1^{\prime}$ spatial resolution.

\subsection{Sub-millimetre Astronomy}

AST/RO, the Antarctic Submillimeter Telescope and Remote Observatory, was a $1.7 \mathrm{~m}$ telescope deployed at the South Pole, in almost constant use from 1995-2005 (Stark et al. 2001). It is the most productive astronomical facility to have operated in Antarctica, if judged by the number of papers produced, with over 50 publications. This is despite its small size, for it was the only telescope in the world then able to access the $350 \mu \mathrm{m}$ window on a regular basis. It was a general purpose facility, able to be used with a range of instruments between $0.2-2 \mathrm{~mm}$, for both astronomical and aeronomy purposes. AST/RO's success was largely attributable to its off-axis design, with the instrumentation placed on an optical table at a warm coudé focus where it could be readily worked on in comfort by the instrument teams. A variety of bolometer and heterodyne systems were used over the lifetime of the facility.

AST/RO was principally used to measure emission lines of atomic carbon (1-0 and 2-1 lines) and carbon monoxide ( $\mathrm{CO} \mathrm{J=4-3}$ and $\mathrm{J}=7-6)$ emitted from molecular clouds in the Milky Way and the Large Magellanic Clouds. These are among the strongest cooling lines from the dense interstellar medium. Results included the first detection of $[\mathrm{CI}]$ emission from the Magellanic Clouds (Stark et al. 1997), the first large-scale maps of [CI] and warm CO from galactic star forming complexes such as Carina (Zhang et al. 2001), NGC 6334 (Kim et al. 2006) and Rho Ophiuchi (Kulesa et al. 2005). The most ambitious project was to map the $[\mathrm{CI}]$ and warm $\mathrm{CO}$ emission from the Central Molecular Zone (the inner $3^{\circ}$ of our Galaxy; Martin et al. 2004; see Fig. 18). The first ground-based spectrum of the [NII] $205 \mu \mathrm{m}$ line was also obtained, emitted from the Carina Nebula (Oberst et al. 2006). This is in the THz spectral region, and shows that, while sites like Dome A are being considered for future $\mathrm{THz}$ telescopes, even at the South Pole this window opens at times.

Sub-millimetre polarization measurements were also made using the SPARO polarimeter on the $2.1 \mathrm{~m}$ Viper Telescope, of the dichroic emission at $450 \mu \mathrm{m}$ from aligned, cold dust grains. This included the detection of a large-scale toroidal field running through the Galactic centre (Novak et al. 2003), and of magnetic fields in four other giant molecular clouds in the Galaxy ( $\mathrm{Li}$ et al. 2006). A statistically significant correlation was found between the magnetic field direction and the orientation of the Galactic plane, suggesting that preservation of the magnetic field direction occurs during the gravitational collapse to produce stars that follows from molecular cloud formation. 


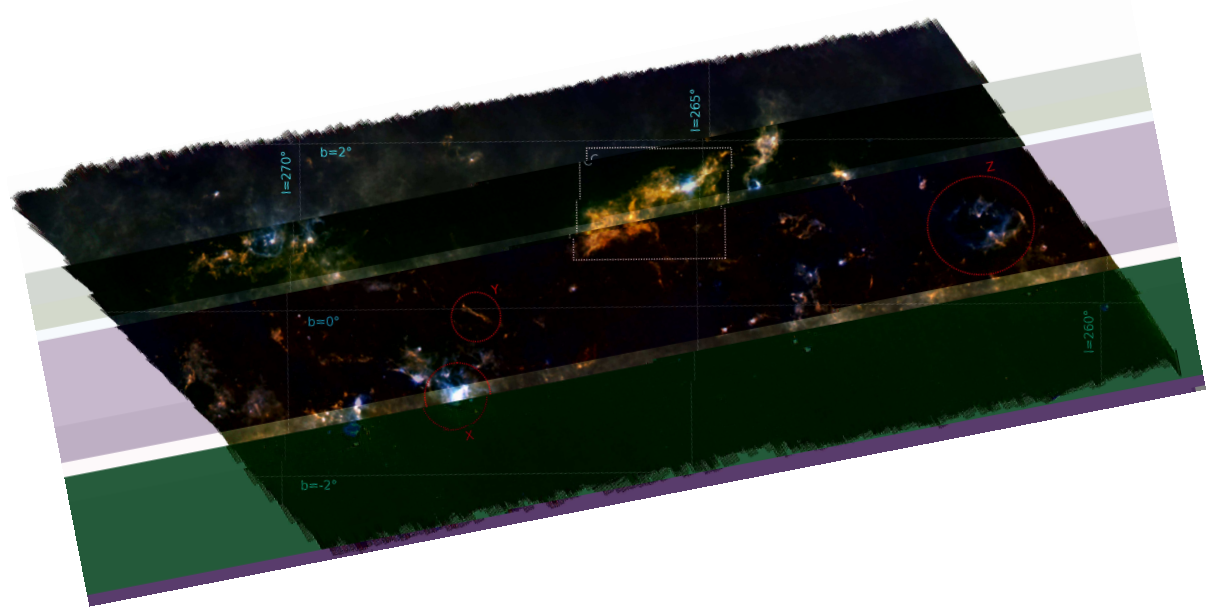

Fig. 19 A sub-millimetre image of Vela Molecular Ridge obtained by the balloon-borne BLAST telescope (Netterfield et al. 2009), launched from the McMurdo Long Duration Balloon Facility. The image shows the complete range of dust cores within the molecular cloud complex, from starless cores to those actively forming stars. The three colours represent emission at $250 \mu \mathrm{m}$ (blue), $350 \mu \mathrm{m}$ (green) and $500 \mu \mathrm{m}$ (red). Blue regions are therefore the warmest gas, typically $\geq 25 \mathrm{~K}$ and red regions the coldest gas $(\leq 13 \mathrm{~K})$. The image covers 50 square degrees and the horizontal lines lie along the Galactic plane, each separated by $b=2^{\circ}$. The angular resolution is $1^{\prime}$.

Sub-millimetre astronomy has also been undertaken in Antarctica on a long duration balloon flight launched from McMurdo. BLAST, the Balloon-borne Large Aperture Submillimeter Telescope, used a $2 \mathrm{~m}$ telescope and bolometer array to survey many square degrees of sky at 250, $350 \& 500 \mu \mathrm{m}$ (Pascale et al. 2008). Following a flight from Sweden in 2005, the dramatic BLAST Antarctic circumpolar flight lasted 11 days in December 2006, as has been spectacularly documented in the movie of the same name. Science programs examined galaxy evolution and the cosmic infrared background, as well as cold dust cores associated with earliest stages of star formation in molecular clouds in our Galaxy. Through measurement of over 500 galaxies from a 9 square degree region, which included the GOODS-S deep field, the sub-millimetre flux-source count relation was determined. From this the cosmic far-infrared background was inferred to be dominated by the emission from individual galaxies, $70 \%$ of the flux coming from known galaxies at $z \geq 1.2$ (Devlin et al. 2009). In our Galaxy, $\sim 50$ square degrees of the Vela Molecular Ridge, $700 \mathrm{pc}$ distant, was mapped to provide a complete and unbiased sample of the dust cores within it, and covering all evolutionary stages in the route to star formation (Netterfield et al. 2009, Olmi et al. 2009; see Fig. 19). Temperatures, luminosities and masses were determined for over 1,000 cores, so providing the core mass function. Around $2 \%$ of the mass was found to reside in cores colder than $14 \mathrm{~K}$, and from this the long inferred lifetimes imply the necessity for non-thermal support mechanisms (against gravitational collapse) to operate at the very earliest stages of star formation. A polarization module is to be added to BLAST for a future flight (Marsden et al. 2008). This will enable the relationship between the magnetic field geometry, and its strength in the cloud cores relative to that in the large scale cloud, to be determined. 
4.4 Infrared Astronomy

The $60 \mathrm{~cm}$ SPIREX Telescope (South Pole InfraRed EXplorer) operated at the South Pole from 1994 to 1999, the first four years with the GRIM 1-2.5 $\mu \mathrm{m} \mathrm{HgCdTe}$ array camera (Hereld 1994), the last two with the Abu 2.4-5 $\mu \mathrm{m}$ InSb array (Fowler et al. 1998). SPIREX was installed just before Comet Shoemaker-Levy 9 struck Jupiter, and was the only telescope in the world with the opportunity of viewing all the impacts. Observation of some of these was affected by poor weather. Nevertheless, SPIREX recorded 16 of the 21 events over the week of the encounter, in July 1994 (Severson 2000), more than any other telescope achieved.

SPIREX's principal achievements were in studying the extensive shells of polycyclic aromatic hydrocarbons, emitting at $3.3 \mu \mathrm{m}$, that are illuminated by far-UV radiation from massive young stars, and in detecting IR excesses from hot dust in disks around pre-main sequence stars. PAHs emission was imaged around the galactic star forming complexes of NGC 6334 (Burton et al. 2000; see Fig 20) and Carina (Brooks et al. 2000, Rathborne et al. 2002), revealing the workings of the galactic ecology in these objects. Thermal-IR excesses, determined from their $[2.2 \mu \mathrm{m}]-[3.5 \mu \mathrm{m}]$ colour, were measured in the low mass star forming regions of Chamaeleon I (Kenyon \& Gomez 2001) and $\eta$ Chaemaeleontis (Lyo et al. 2003), and in the high mass star forming regions of 30 Doradus in the LMC (Maercker \& Burton 2005) and RCW57/NGC3576 (Maercker, Burton \& Wright 2006). A high disk fraction was found in all cases, including, interestingly, for massive as well as for low mass stars.

SPIREX demonstrated that the projected sensitivities for the IR, based on the measured sky background drop, could indeed be achieved. A summary of all the science results obtained with SPIREX is given by Rathborne \& Burton (2005). At the time of the observations (1998), the measurements of 30 Doradus made with SPIREX were the deepest imaging that had been obtained at $3.5 \mu \mathrm{m}$, achieving a sensitivity of 14.5 mags. in 9 hours of on-source integration. However, the small size of the facility (especially in comparison to $8 \mathrm{~m}$-class facilities now available), combined with the depth of the surface boundary layer at the South Pole (necessitating the use of wavefront correction systems to obtain the free-air seeing), has stalled the development of IR astronomy in Antarctica since SPIREX was de-commissioned.

With the opening of Concordia Station at Dome $\mathrm{C}$ and the construction started of Kunlun station at Dome A, both with their thinner surface boundary layers than South Pole, infrared astronomy may now move forward again in Antarctica. An $80 \mathrm{~cm}$ mid-IR telescope, IRAIT (the International Robotic Antarctic Infrared Telescope), has been built by the University of Perugia (Busso et al. 2010). It has been equipped with the 2-28 $\mu \mathrm{m}$ AMICA imager (Dolci et al. 2010) and is currently being installed at Dome C.

\subsection{Optical Astronomy}

A limited number of optical astronomy experiments have been conducted in Antarctica, generally as part of site testing programs. As described in 3 the $5 \mathrm{~cm}$ SPOT telescope was used at the South Pole to obtain 1 continuous week of data monitoring the light curve of the Wolf-Rayet star $\gamma^{2}$ Velorum (Taylor 1990). In 2.4 the ASTEP $(10 \mathrm{~cm}$ telescope; Crouzet et al. 2010) and sIRAIT ( $25 \mathrm{~cm}$ telescope; Strassmeier et al. 2008) experiments at Dome C were described, as well as the $14.5 \mathrm{~cm}$ CSTAR telescope at Dome A (Zou et al. 2010). These latter three experiments undertook a variety of sky brightness, transparency and photometric 


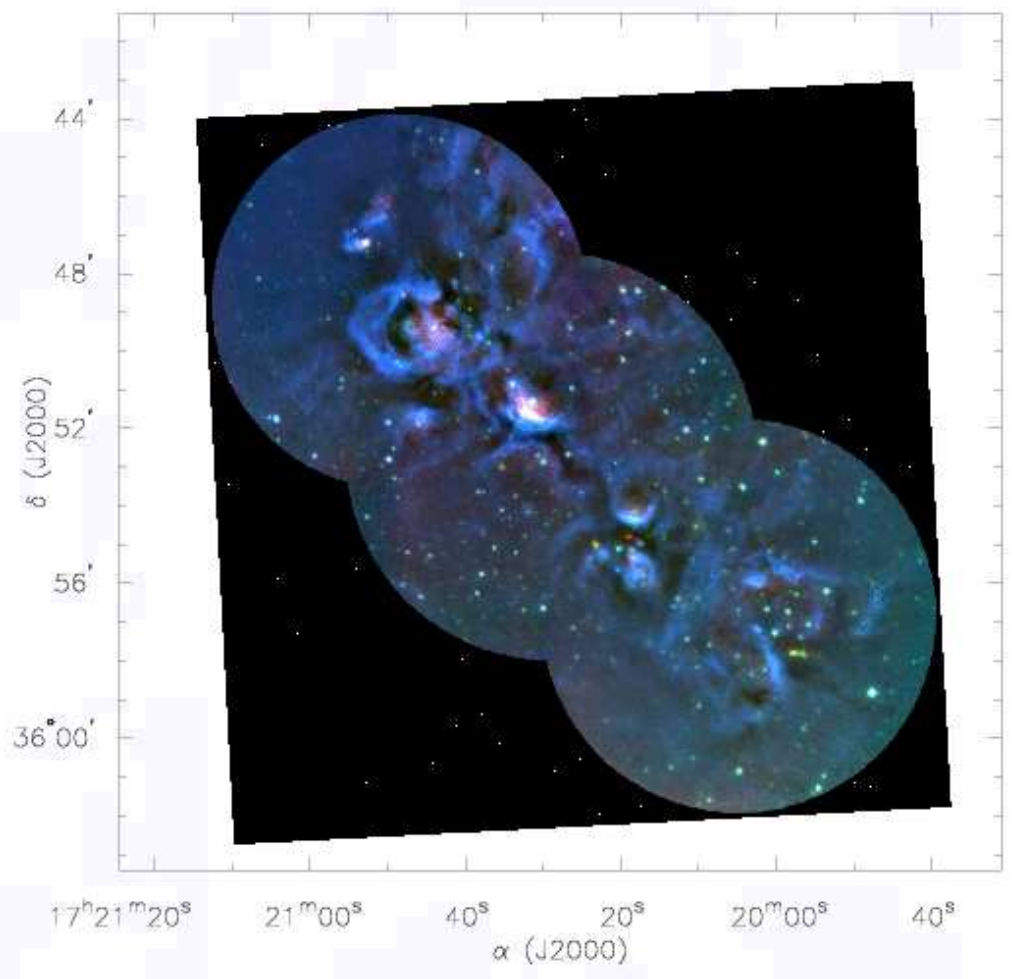

Fig. 20 An image showing the polyaromatic hydrocarbon (PAH) emission at $3.3 \mu \mathrm{m}$ that envelopes the NGC 6334 star forming complex, as imaged by the infrared SPIREX telescope at the South Pole (Burton et al. 2000). Dark dust lanes (black) and deeply embedded massive protostars (red) are evident, surrounded by shells of PAHs (blue), excited by the far-UV radiation from the most massive young stars, each separated by $\sim 1 \mathrm{pc}$ along the dust ridge. The image covers a $30^{\prime}$ region, with a spatial resolution of $1.5^{\prime \prime}$. The three colours represent $3.3 \mu \mathrm{m}$ PAH (blue), $3.5 \mu \mathrm{m} \mathrm{L}$-band continuum (green) and a $1 \%$ narrow band filter at the wavelength of the $4.05 \mu \mathrm{m} \mathrm{Br} \alpha$ line. 
monitoring observations, in the case of CSTAR also resulting in a catalogue of some 10,000 stars in a field centred on the South Celestial Pole (Zhou et al. 2010).

\subsection{High-Energy Astrophysics}

High-energy astrophysics in Antarctica began with the installation of two muon telescopes for cosmic ray detection at Mawson Station in 1955. The Mawson observatory later contributed to the experimental verification of the spiral nature of the solar magnetic field long before direct measurements could be made by satellites (McCracken 1962). Cosmic ray research is today pursued at a number of locations around Antarctica, most notably Mawson, McMurdo and the South Pole (see Duldig 2002). Equipment for its conduct includes cosmic-ray detectors, neutron monitors and muon telescopes.

The GASP gamma ray telescope at the South Pole sought to find cosmic sources of gamma rays via the Cherenkov light produced by cosmic rays accelerated by the interaction of gamma rays with nuclei in the atmosphere (Morse \& Gaidos, 1990). This search is assisted at the South Pole by the long polar night and constant source zenith angle. The experiment was not successful, however, in finding any gamma ray sources. It led to the construction of the SPASE air shower array at the South Pole, increasing the effective collecting area for detecting such events. SPASE, the South Pole Air Shower Experiment (Smith et al. 1989), also failed to find sources of gamma rays, with the particle events recorded showing an isotropic distribution across the sky (van Stekelenborg et al. 1993). This was followed by SPASE-2, an enhanced array (Dickinson et al. 2000), which was built on the ice-surface above the AMANDA neutrino array (see below) so that it could also work in conjunction with it. The objective was to measure the particle compositions in air showers in the TeV range (Ahrens et al. 2004). Though SPASE-2 again failed to find gamma ray sources, this detection technique has now matured with the success of the HESS gamma ray telescope in Namibia, through stereoscopic imaging of the air showers in the atmosphere (e.g. Aharonian et al. 2004).

AMANDA, the Antarctic Muon And Neutrino Detector Array (Andrés et al. 2000, 2001), was the first experiment at the South Pole to search for cosmic sources of neutrinos. The technique uses photomultiplier tubes (PMTs) placed into holes drilled in the ice, extending from several hundred metres to three kilometres deep. The PMTs detect Cherenkov radiation resulting from the exceedingly rare encounters of neutrinos with ice or rock nuclei. The PMTs point downwards to shield the detectors from the vastly greater fluxes from downward-travelling muons (produced by the interaction of cosmic rays in the atmosphere). Hence the array serves to detect upward-travelling neutrinos that have passed through the Earth, entering it from the northern hemisphere. With its extension, AMANDA-II, over 600 hundred neutrinos were detected, though no statistically significant cosmic sources of neutrinos were found in the data set (Ackermann et al. 2005).

AMANDA served as the prototype for IceCube, the largest science experiment yet to take place in Antarctica (Ahrens et al. 2004). Almost complete (see Fig. 21), IceCube uses a cubic kilometre of ice as its detector, the volume calculated as necessary for it to operate as a true telescope, able to image sources of cosmic neutrinos. When completed 80 strings, each containing 60 digital optical modules, will have been suspended in the ice. Each string extends from 1.5 to $2.5 \mathrm{~km}$ beneath the surface, and they are spread out over a square kilometre, creating a cubic kilometre of collecting volume. The array is optimised for detecting highenergy neutrinos, $\mathrm{E}_{v}>\sim \mathrm{TeV}$, with a closer-spaced core for lower energy neutrinos. Over the first 6 months of operations $\sim 7,000$ upward-travelling neutrinos were recorded from 


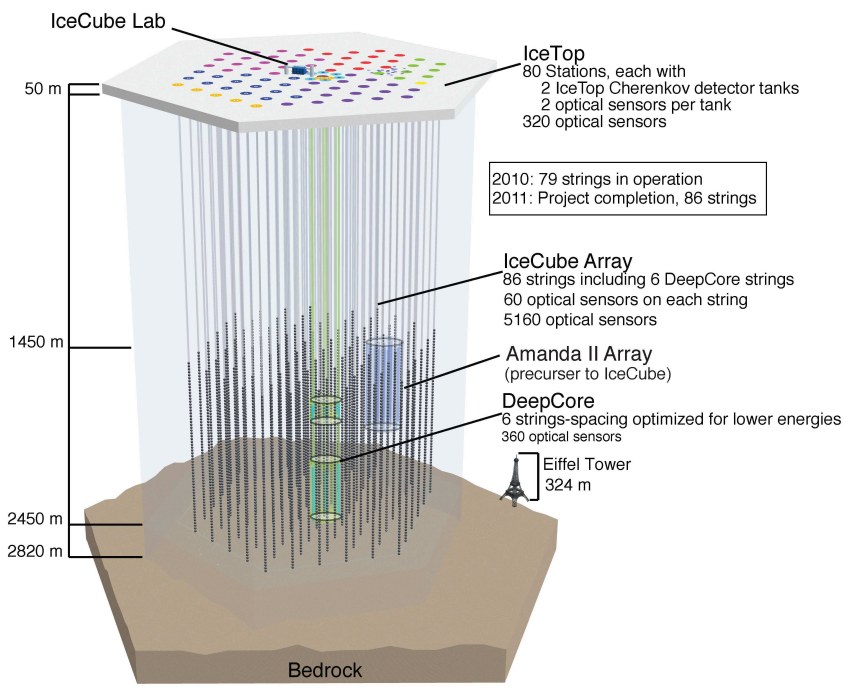

Fig. 21 A sketch illustrating the construction of the IceCube neutrino observatory at the South Pole, together with the Eiffel Tower to provide a size comparison. 80 strings, each $1 \mathrm{~km}$ long, are buried from 1.5-2.5 km deep in the ice (reaching close to the bedrock beneath the ice), together with a closer spaced core at the centre. 60 digital optical modules are strung along each string. At the ice surface is the IceTop experiment, together with the observatory laboratory. Cherenkov radiation from muons generated by the rare neutrino interactions with nuclei in the ice creates a cone of light that can be detected by the optical modules lying within its path, so allowing the direction of the exciting neutrino to be inferred. Credit: IceCube Consortium (icecube.wisc.edu).

the first 40 strings deployed (Filimonov et al. 2010). No statistically significant sources of neutrinos can yet be discerned in this data set.

Another experiment at the South Pole is also seeking to find ultra-high energy $\left(E_{v}>\sim\right.$ $\mathrm{PeV}$ ) neutrinos - RICE, the Radio Ice Cherenkov Experiment - through measuring coherent pulses of radio Cherenkov emission produced from neutrino-induced cascades passing through the transparent ice (the Askaryan effect; Askaryan 1965). The radio receivers used 
for this have been placed in some of the AMANDA boreholes. The status of the experiment, and the neutrino limits obtained, are discussed further in Kravchenko et al. (2008).

The final neutrino experiment underway in Antarctic is ANITA, the Antarctic Impulsive Transient Antenna (Barwick et al. 2003). This is flown $\sim 35 \mathrm{~km}$ above the continent on a long-duration balloon flight from McMurdo Station, and seeks to measure coherent radio pulses from $\mathrm{EeV}\left(10^{18} \mathrm{eV}\right)$ neutrino interactions. These are produced by a compact plug of relativistic particles, just a few centimetres in diameter, but the experiment makes use of the elevation of the balloon to search for these over $\mathrm{a} \sim 1,000 \mathrm{~km}$ extent of the Antarctic ice sheet - an effective collecting area of one million square kilometres! No neutrinos were detected from either the first 35 day flight in 2006-2007 (Gorham et al. 2009) or from a second, more sensitive, 31 day flight in 2009 (Gorham et al. 2010), but strong limits have been set for the flux of cosmic neutrinos in the $10^{18-21} \mathrm{eV}$ range.

As described in $\$ 3$ an ice core drilled at Dome $F$ has been used to infer that gamma rays produced by two historical supernovae also produced ionization events in the Earth's atmosphere (Motizuki et al. 2010).

\section{Conducting Science in Antarctica}

\subsection{Life for the Antarctic Scientist}

In Antarctica logistics determines what it is possible to do. Without pre-existing infrastructure and support capability, conducting frontier science is impossible. While it may still be possible for an adventurer to forge new routes across the Trans Antarctic Mountains, the age of the Antarctic hero is long over. The exploits of Amundsen, Scott, Shackleton, Shirase and Mawson may have inspired a fascination with Antarctica, but they do not provide a model for today's explorer in the continent. For the modern explorer the challenges lie elsewhere, in making sophisticated instrumentation work in conditions very different to the laboratory back home. Life in a modern Antarctic station is certainly no luxury experience, but it is not arduous either. One might have to share rooms for sleeping, limit showers to two minutes, twice a week, and deal with high-altitude acclimatisation, but these merely serve to make a person slightly uncomfortable. Dealing with continuous daylight in summer can be more of a problem, but this is only a matter of upsetting circadian rhythms.

Antarctic scientists working on a modern station do not need to worry about cooking their meals, shopping for food or commuting to work. The essentials of daily life are all provided. This is a necessity of safe working practice in Antarctica. For, despite modern conveniences, the dangers of Antarctica remain real, even if they are not the constant threat the early explorers faced. Nations conducting Antarctic science go to great lengths to provide facilities that are safe and practical for their inhabitants. For every scientist present, four or five people are there to support them, and keep them alive and happy.

The Antarctic scientist is left to concentrate on getting their experiment working. This is not to say that life is without its concerns. In summer a base is generally overcrowded. Work space is limited and may need to be regularly negotiated for. Daily timetables can be subservient to the operational needs of the station. There is little time for leisure, and waking hours are consumed by the requirements of work. With constant day light and the extremely dry air, sleeping can be difficult. A person can be in a state of permanent stress. It takes five minutes to get dressed every time one goes outside. Antarctica, and your immediate surroundings, become the real world. Life back home seems like a dream. Indeed, for an increasing number of people who winter-over from year to year, it does become a dream! 
Wintering is another matter and the experience can only properly be described by someone who has done so (the author has not). Six months without the Sun at the Pole is a very long time, though it is not actually dark for all that time. Twilight lasts for a month. When the Moon is up it is easy to see across the ice. But the cold is much harder to endure in winter than it is in summer. Skiways are not maintained, and the daily walk to work over the snow can become a feat of endurance. The only company is that of the other winter-overers. They are the only people you will see for eight months. While this can be a source of intense friendships, it can also lead to strong inter-personal problems, for it is impossible to avoid a person's company. Many do find themselves counting out the "days" to the first flight out in the Spring.

However, in one important way life has become much easier for the winter-overer, and that is through communications. No-one is truly alone anymore through the winter. The daily email torrent does not stop. The internet is there to browse, news from around the world continues to arrive, phone calls can be made. Principal investigators ring in to discuss a problem with an experiment. Webcams let people back home keep tabs on both experiment and experimenter. Conference calls and internet video conferencing are arranged among members of the research team, scattered around the globe. Science can be done and papers written. In extreme circumstances it is even possible to get out of Antarctica in a medical emergency.

This is not to say that winter-overers don't go through periods of depression. In the heart of winter some still become hermits, locking themselves away in their rooms, watching videos for much of the day, neglecting their work. But this happens less than it did. The converse is also true. Some scientists are so involved with their work, and in their daily correspondence with colleagues, that they seem to slot straight back into their previous occupation when they get off the "Ice". Yet it is also true to say that for most winter-overers it is the transition back to normal life that is the hardest aspect to deal with, not the winter itself.

A final observation about working in Antarctica. It is not as expensive as might have initially been expected, at least for small-scale experiments. When a scientist arrives in Antarctica everything needed to support their daily life has already been paid for. For the scientist, their job is to bring the working experiment to the appropriate mainland departure point. From there, everything else is done by the Antarctic programs of the nation(s) they are working with, to bring them to their destination and then support them there. Scientists do not pay for their deployment or living costs in Antarctica. The situation is akin to the user of a space telescope - they do not have to factor in the launch costs of the spacecraft or the operation of mission control when planning their experiment. These costs are subsumed within the budgets of national programs. Essentially a decision has already been made by nations to support personnel in Antarctica and so this is budgeted for. The processes which determine what experiments are to be supported also determine the available personnel slots that the national Antarctic program will support. If your program is good enough to be rated for deployment, that deployment is already paid for. You simply have to find the funds to have your medicals, get yourself to the point of departure, and of course build your experiment.

\subsection{Experimental Challenges}

While technology makes working in Antarctica increasingly easy, it never will be the same as in the lab back home. Careful planning ahead is essential. Never try to commission an instrument for the first time in Antarctica, always test it before deployment. This is, of course, 
wise practice anywhere, but essential in Antarctica. Though, given the many competing demands most scientists face at their home institutions, this is easier said than done. It is not a total disaster, however, if something breaks. Stations generally have extensive supplies of parts. Station personnel are inventive when called upon to find something they don't have. Machine workshops can often be used to re-make a broken part. Even if not, it is still possible to order a replacement, at least in summer. At the South Pole items can generally be flown in within the week.

As discussed further by McGrath et al. (2008), unique operational aspects of an Antarctic observatory arise from its remoteness, the polar environment and the unusual observing cycle afforded by long periods of darkness and daylight. A telescope must be designed for remote observing via satellite communications, and must overcome both limited physical access and data transfer rates. Commissioning and lifetime operations must deal with extended logistics chains, continual wintertime darkness, extremely low temperatures and frost accumulation.

One of the most difficult issues for many astronomical applications has been the supply and maintenance of cryogens, especially liquid helium, which must be flown in from the mainland. Even with just a $1 \%$ evaporation loss per day, by mid-winter a helium supply would be exhausted, so halting sub-millimetre and CMBR experiments, for instance. Fortunately, with the continued development of closed-cycle systems for instruments, this should become a problem of the past.

The design challenges caused by the extreme cold are dominated by the continued performance of lubricants, the mechanical clearances that may change due to thermally induced dimensional changes, and the operation of electronics designed for a room temperature environment. However, all of these issues are quite readily overcome with proper mechanical design, so long as it takes account of the temperature requirements. The high rates of change of temperature that can take place within the boundary layer can be more problematic, especially for components with high thermal mass and tight thermal equilibrium requirements notably, the primary mirror of a telescope.

In addition to the debilitating effects on image quality caused by turbulence, the surface layer also poses an engineering challenge for two further reasons. The first is the large vertical temperature gradient that can occur within it (up to $\sim 1 \%$ at surface level and even $\sim 0.15^{\circ} / \mathrm{m}$ at $30 \mathrm{~m}$ at Dome C), especially on the most stable days in winter. The second is the super-saturated humidity within the layer, which readily leads to icing on exposed surfaces. To solve both these problems, a temperature and humidity-controlled enclosure for a telescope may be required (e.g. see Saunders et al. 2008a). Such an enclosure (e.g. see Fig. 22 could be continuously flushed with sub-saturated air, matched in temperature to the external air at the dome aperture. This air is drawn from closer to the surface of the snow (where it is colder, and so drier). It could be heated using waste heat from the instrumentation, resulting in its humidity falling below the saturation point. In addition, such a scheme could deliver excellent dome seeing, as the temperatures could be closely matched and venting conducted so that the external airflow suffers minimal disruption.

There is also a need to consider how to provide electrical power for any significant astronomical facility, which might require several tens of $\mathrm{kW}$. This might not readily be expected to be drawn from a station diesel power supply. However solar energy (in summer) and wind power (the turbine mounted on a tower to place it above the boundary layer, where a steady wind blows) could provide a clean energy source for observatories. As discussed by McGrath et al. (2008), this only imposes a modest addition to the total cost of a facility. A clean energy source may also be necessary to gain public support for a new facility, so minimising pollution and maintaining the pristine condition of the environment. In particular, 


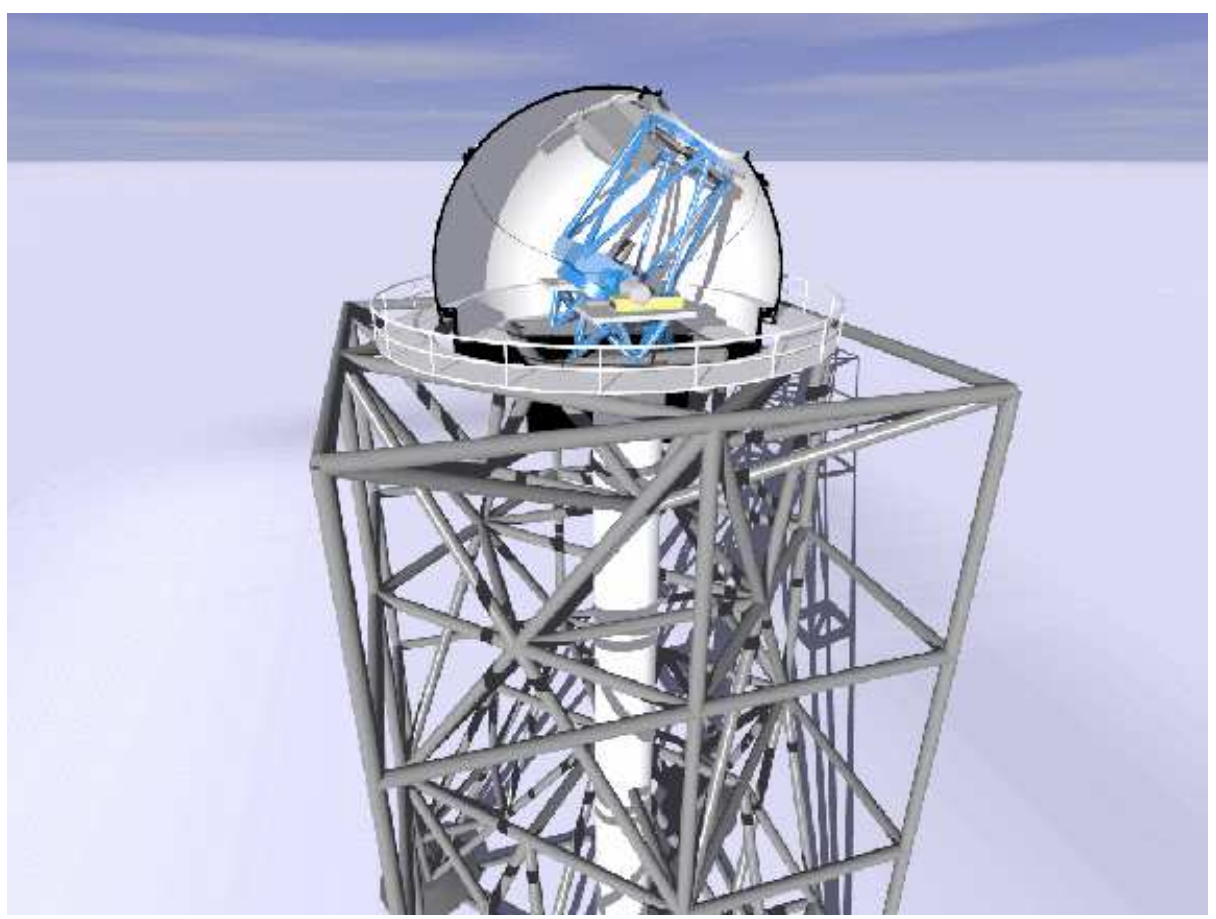

Fig. 22 A concept design for the $2.4 \mathrm{~m}$ PILOT optical-IR telescope (Saunders et al. 2008a). The design reflects a temperature and humidity controlled callote-style enclosure for the telescope, placed on top of a stiff tower that raises it above the surface boundary layer. Cold air from close to the ice surface is drawn into the enclosure and warmed to the ambient temperature at telescope level, thereby lowering its humidity below the frost point. Careful venting of the air to the atmosphere maintains the superb free air seeing. Image by Andrew McGrath, Anglo Australian Observatory.

it would minimise contributions from locally produced aerosols (e.g. from the exhausts of diesel generators), important for maintaining the stability of the local sky emission.

\subsection{Antarctic Politics}

Antarctic politics is unique, truly in a world of its own. While on a national level local issues may determine how a country runs its Antarctic program and funds its science, on an international level Antarctic affairs are matters for co-operation. The governance of Antarctica is through the Antarctic Treaty 3 , first signed by 12 nations in 1959, and now with 45 signatories attached to it. Of these, 28 nations are consultative members, which means that they have voting rights, a status attained through the conduct of sustained scientific research in Antarctica. 29 nations operate field stations, 19 of which run the 36 year-round stations. The Treaty bypasses issues of sovereignty by not recognising any territorial claims made while the Treaty is in force. It states that Antarctica is to be used for peaceful purposes only, with scientific investigation and collaboration specifically encouraged. The Antarctic Treaty is in fact a remarkably short and simple document comprising just 14 articles. Seven nations have

\footnotetext{
${ }^{3}$ See http://www.scar.org/treaty/at_text.html
} 
laid claim to parts of Antarctica (Argentina, Australia, Britain, Chile, France, Norway and New Zealand), with Argentina, Britain and Chile having overlapping claims. The USA, on the other hand, has not made a claim, but has reserved the right to do so. The Treaty avoids any issue over these claims by neither recognising them nor denying them. Any country (or indeed person) is free to go anywhere in Antarctica without restriction, assuming they have the means to do so.

A measure of the Antarctic Treaty's success is that there remains a large sector of West Antarctica that has not been claimed by any nation. The Treaty was further strengthened in 1991 by the adoption of the Madrid Protocol. This defines Antarctica as a natural reserve devoted to peace and science, and specifically forbids any mineral activity, except for research. It also strengthened measures for the conservation of flora and fauna, and set up standards for waste management.

\subsection{SCAR and the IAU}

SCAR, the Scientific Committee for Antarctic Research, is the peak body for Antarctic affairs. Established in 1957, as a committee of ICSU, the International Council for Science, SCAR is charged with the initiation, promotion and co-ordination of scientific research in Antarctica. Within SCAR there are 31 Full Members (countries with active research programs in Antarctica), 4 Associate Members (countries without independent research programs in Antarctica) and 9 Union Members (those ICSU scientific unions with an interest in Antarctica research). The IAU (International Astronomical Union) is one of these Union Members.

Operating alongside SCAR are a number of bodies that have been set up by further international treaties in order to manage the conservation of flora and fauna in and around Antarctica, including particularly that in the Southern Ocean. SCAR holds an open science congress every two years, which also serves as the major forum bringing together the managers of national Antarctic programs. Through a motion put forward at its $22^{\text {nd }}$ congress in Rome in 1994, SCAR passed a resolution recognising the scientific value of Antarctic astronomy and calling for the development of the field.

Like the IAU, SCAR has numerous divisions within it. There are three Standing Scientific Groups (SSGs), covering Geo Sciences, Life Sciences and Physical Sciences. There are also five Scientific Research Programs (SRPs), which have a focus on international scientific co-ordination. In 2010 Astronomy became one of these programs - Astronomy and Astrophysics from Antarctica (AAA $\sqrt[4]{4}$ - replacing the ICESTAR program (involving solarterrestrial \& aeronomy research). John Storey, of the University of New South Wales, has been appointed the first Chief Officer of AAA, which has set for itself several goals (see Storey, 2010). These involve the coordination of site testing activities (including related activities in the Arctic) and the provision of publically accessible data bases for their data, as well as improving cooperation with other physical sciences. They also look to future development: defining and prioritising science goals, creating a roadmap for developing future astronomical facilities and stimulating international cooperation to construct and use those facilities. To undertake this work AAA has been structured into four themes, covering site testing, the Arctic, science goals and major facilities.

The International Astronomical Union also supports the development of the Astronomy in Antarctic. At the IAU's $21^{\text {st }}$ General Assembly, in Buenos Aires in 1991, a working

\footnotetext{
${ }^{4}$ See www.phys.unsw.edu.au/jacara/AAA_SRP_webpage/.
} 
group Encouraging the International Development of Antarctic Astronomy 5 was formed, with Peter Gillingham of the Anglo Australian Observatory as its first chair. The author is the current chair. The IAU passed a resolution at that General Assembly encouraging the development of the then fledgling field. The Working Group is under the auspices of two IAU Divisions (Division IX on Optical \& Infrared Techniques and Division X on Radio Astronomy). There have been regular sessions on Antarctic astronomy at subsequent IAU General Assemblies (see Gillingham (ed) 1992, Burton (ed) 2005b, 2007, 2010b), as well as several international conferences and workshops devoted to aspects of the field.

\subsection{Funding for Antarctic Astronomy}

SCAR and the IAU serve principally as fora where scientists can come together to discuss progress and plans for their fields of endeavour. They do not fund Antarctic science, however, for that is the province of nations, acting either individually or collaboratively.

The funding process itself, for deciding upon and supporting Antarctic science, differs considerably between nations. These have evolved according to the Antarctic traditions and histories within individual countries, to differing national priorities, including whether a nation has made territorial claim to the continent, and to the different cultures surrounding science funding. In some countries Antarctic science is confined mainly within their national Antarctic agency, with both the logistics and science conducted by that agency. In other countries, science funding is separated from the logistics of operating the bases. More commonly, a mix of the two exists (see Burton, 2005).

While the peer review process might be followed for determining on science priorities, this is often contained within pre-defined government goals for Antarctica. These can serve to support current activities but make it difficult for new ones to emerge. This has created difficulties for furthering astronomy in Antarctica in many nations, for astronomy is not a traditional Antarctic science. It can be excluded from a national Antarctic program by falling outside those goals, and so need to seek support from other funding agencies. These agencies are, quite often, resistant to funding research in Antarctica as they argue that it should be funded by the national Antarctic program. With no agency clearly responsible for deciding upon funding for astronomical ventures in Antarctica, it can be difficult to develop astronomical programs in Antarctica beyond relatively low-cost site testing activities, despite the well-recognised strength of many of the science cases that have been made. Those nations that have managed to resile this conflict, between supplying the logistics and deciding upon the science, have managed to do the best Antarctic science, regardless of the scientific discipline. No where is this success more apparent than in the exotic range of science now evident at the South Pole, nurtured slowly over three decades of planning and pursuit.

Despite these differences between funding policies of nations, collaborative activity forged between scientists can be a spontaneous event, with national boundaries playing little role. One of the delights of working as an Antarctic scientist is the relative ease with which international collaborations can be made. If you can devise an experiment that will be advantageous to conduct at the station of another country, that country can generally be persuaded to support you. Of course, it needs to be logistically possible to set-up and operate the experiment. There also need to be scientists from the host country supporting the project, and it will have to pass through an assessment process to determine its relative standing among other projects. But once these steps are taken, the nation running the base will take it there,

\footnotetext{
5 See www.phys.unsw.edu.au/jacara/iau.
} 
and provide the logistics to install the experiment, all without charge. It is up to the experimenter to find the funds and personnel to build the experiment in the first place, but most of the operations costs become subsumed in those of the station. The science, of course, needs to be shared among all the partners involved.

\section{Antarctic Astronomy Tomorrow}

\subsection{Future Science}

Extensive science cases have been written for astronomy in Antarctica, a diverse range of facilities proposed, funding sought for some of them and roadmaps prepared for future developments. Yet any words written today, about what astronomy will be done in Antarctica tomorrow, will almost certainly contain more speculation than fact. This section therefore will only briefly discuss the many ideas that are current, and try to draw together common themes that indicate likely future directions.

Some areas of science have been well served in Antarctica, with potent science cases leading to funded facilities, following staged development programs which will continue over the coming decade. This clearly is the case with the fields of CMBR and neutrino astrophysics. Both are characterised by a series of increasingly sensitive experiments, though for the former these have yielded detections of subtle effects while for the latter non-detections. Yet both fields have essentially followed the path expected of them by the quantitative predictions underpinning their science.

Traditional photon astronomy has, however, not been so well served by Antarctic astronomy, with only modest-sized facilities built so far for observations in the infrared and sub-millimetre. We therefore consider here some of the science and facilities envisaged for the thermal-IR and $\mathrm{THz}$ portions of these spectral regimes.

Infrared Astronomy A series of science cases have been developed for Antarctic facilities, focussed largely on the prospects for infrared astronomy given the greater sensitivity of an Antarctic telescope over an equivalent-sized mid-latitude facility (e.g. Burton et al. 1994, Burton et al. 2001, Burton et al. 2005, Lawrence et al. 2009a, 2009b, 2009c). These science cases have concentrated on intermediate-scale facilities (i.e. $2 \mathrm{~m}$-class telescopes), perceived as the most likely size for the next Antarctic IR telescope. The capabilities of IR facilities elsewhere, both on Earth and in space, have improved dramatically over this period. So too has the science focus for prospective Antarctic facilities of this scale changed as this has occurred. It has narrowed into the parameter space where modest Antarctic facilities may still compete with the best facilities available elsewhere - that science which requires wide-field, high spatial resolution imaging in the near-infrared and (spectroscopically) the mid-infrared. This science is still impressive, however, even for a modest-size telescope. It includes such projects as searching for the first light in the Universe via pair-instability supernovae and from IR-only emitting gamma-ray bursts, as well as deep $2.4 \mu \mathrm{m}$ imaging surveys looking for the first galaxies to evolve in the universe. Exo-planet searches would also be well facilitated, carried out both through the transit technique and through microlensing. It would also be possible to image the warm molecular gas of the Galaxy directly, on the arcsecond scale, as opposed to the arcminute scale available in the millimetre, by measuring the $17 \mu \mathrm{m}$ line of molecular hydrogen, the ortho-ground state transition of the molecule which is sensitive to this environment. No Antarctic telescope has been funded yet that could undertake any of these investigations, though several have been proposed as we 
discuss further in $\S 6.2$ (e.g. AIRO at the South Pole, PILOT/PLT at Dome C and KDUST at Dome A).

Terahertz Astronomy Even at the best temperate latitude sites such as the Chajnantor plateau in Chile there is only occasional access to the THz windows. Only from Antarctica could substantive $\mathrm{THz}$ surveys be considered, other than from space. Two of the most important cooling lines in the dense interstellar medium emit in the $\mathrm{THz}$ regime; [CII] at $1.9 \mathrm{THz}$ $(158 \mu \mathrm{m})$ and $[\mathrm{NII}]$ at $1.5 \mathrm{THz}(205 \mu \mathrm{m})$. These lines provide tools to probe the formation and life-cycle of interstellar clouds. A THz telescope has been funded for Antarctica, the $0.8 \mathrm{~m}$ Stratospheric THz Observatory (STO; Walker et al. 2008). This is to be flown from a long duration balloon scheduled for launch from McMurdo in the 2010/11 austral summer. STO aims to survey $\sim 30^{\circ}$ of the Galactic plane for emission from these species, with a spatial scale of $1^{\prime}$. Further ahead, there are plans for much larger $\mathrm{THz}$ facilities at Domes $\mathrm{A}$ and F, capable of extending observations to extra-galactic sources. The estimates made for the THz transparency (Yang et al. 2010) at Dome A suggest that the [NII] line could be measured there, with $>28 \%$ transmission, for a quarter of the time. At Ridge A these same estimates suggest that even the [CII] line will be accessible, for perhaps $10 \%$ of the time.

\subsection{Future Astronomical Facilities in Antarctica}

In this section we discuss briefly discuss some of the plans that have been formulated for new facilities on the Antarctic plateau.

South Pole The IceCube neutrino telescope dominates the logistics of supporting the South Pole station. Completing this facility so that it can go into full operation is a priority for the next few years. The existing CMBR facilities at Pole will continue to operate, building their data sets over several years so as to be able to address their science goals, as outlined in 4.2 This includes the QUaD experiment on DASI (E-mode polarization mapping), BICEP (B-mode detection) and the SPT (SZ-effect in distant galaxy clusters). Once this last survey is completed, the plans for the SPT are to use it for conventional sub-millimetre astronomy, building upon programs started with the AST/RO telescope. SPT will be capable of undertaking surveys at the highest frequencies reached by ALMA, as well as conducting $\mathrm{THz}$ observations when conditions permit. While a $2 \mathrm{~m}$ class infrared telescope was once proposed for the South Pole (AIRO; Jackson 2001), this was before the first measurements of seeing were obtained from Dome C. An infrared telescope is not currently on the agenda for the South Pole. On the other hand, a $2 \mathrm{~m}$ optical/UV telescope, ACWI - the Antarctic Cosmic Web Imager - is being developed. This will be used to search for red-shifted UV resonant line emission (Ly $\alpha$ 121.6nm, CIV 155nm \& OVI 103nm; Moore et al. 2008b) from the intergalactic medium at $z \sim 2-3$, in order to study large-scale structure and the dark matter distribution (i.e. the Cosmic Web). The technical driver is the extremely accurate sky subtraction that should be attainable due to the combination of low extinction (resulting from the low aerosols) and long duration observations at constant zenith angle, so minimising instrumental systematics (high image quality is not required for this application).

Dome $C$ Astronomical activity at Dome $\mathrm{C}$ to date has consisted of site testing and several small-scale prototype facilities, that will culminate with the operation of the $80 \mathrm{~cm}$ midinfrared IRAIT telescope. Consideration of future plans for facilities at Concordia station 
has been conducted under the auspices of the European Union-funded ARENA consortium, a network program run from 2006-09. Each of the three yearly conferences resulted in a book summarising the deliberations over the previous year, evolving as knowledge of the site conditions and technical challenges grew (Epchtein \& Candidi 2007, Zinnecker, Epchtein \& Rauer 2008, Spinoglio \& Epchtein 2010). ARENA finished its activities by producing a roadmap for future development at Dome C (Epchtein et al. 2010). This was organised through 6 working groups, who considered the prospects at Dome C for infrared, sub-millimetre, interferometric, time-series, CMBR and solar science, respectively. ARENA see the development of a $2 \mathrm{~m}$ class, wide-field infrared telescope as the next stage in the development of Dome C (see Burton et al. 2010), such as that based on the design study conducted for the PILOT / PLT telescope (see Storey et al. 2007, Saunders et al. 2008a, 2008b). Funding is being sought for a Phase B study for this concept, before construction could start.

Dome $\mathrm{C}$ is clearly an excellent site for many photometric monitoring experiments, for sub-millimetre and solar telescopes, for CMBR measurements and for infrared interferometers. The ARENA roadmap considers a staged development of Concordia station to support all these types of facilities.

Dome A Although the construction of Kunlun station at Dome A has only recently begun, China has drawn up plans for several major astronomical facilities, under the auspices of the Chinese Center for Antarctic Astronomy (Gong et al. 2010, Cui 2010). These include a three telescope array of $0.5 \mathrm{~m}$ Schmidt telescopes (AST3) to search for transiting exo-planets and obtain light curves of supernovae, a $4 \mathrm{~m}$ infrared telescope (KDUST; Zhao et al. 2010) to search for distant supernovae and distant galaxies, and a $10 \mathrm{~m}+$ diameter sub-mm / THz telescope. A further automated observatory (PLATO-A), with increased power capacity, is also planned for Dome A.

Dome F While site testing at Dome F has barely commenced, it is clear the site will also be exceptional for observational astronomy. Site testing is envisaged to continue, making use of another PLATO-style automated observatory, as at Dome A. Two prototype projects are under construction for future deployment there, a $40 \mathrm{~cm}$ infrared and a $30 \mathrm{~cm} \mathrm{THz}$ telescope (Ichikawa 2010). Two facilities have been proposed after this, an ultra light weight $2.5 \mathrm{~m}$ infrared telescope (Takato et al. 2008) and a $10 \mathrm{~m}$ class THz telescope. The infrared telescope would undertake deep surveys for galaxies at $2.4 \mu \mathrm{m}$, and the $\mathrm{THz}$ facility examine dusty galaxies to study galaxy evolution at high redshift.

Ridge A As argued by Saunders et al. (2009) this may be the best site on the planet for THz astronomy. No stations are as yet envisaged for Ridge A, however, nor are any overland traverses planned to the site. Site quantification may have to take place through Twin Otter deployment of an automated observatory, such as using a modified, light-weighted version of the PLATO module.

\subsection{Final Words}

Antarctica is a land of extremes. Descriptors such as coldest, driest, highest, windiest and calmest can all be applied to the continent. It is barely a century since the first explorers ventured into the interior. The Antarctic plateau has been found to provide the pre-eminent conditions for making many kinds of astronomical observations from the Earth. Antarctica 
remains a challenging place to work, but technology now provides for ready access to the interior of the continent, as well as for sophisticated scientific experiments to be carried out.

Human perceptions of the continent have not, however, caught up with recent accomplishments in Antarctica. While the 'heroic age' of Antarctic exploration is long over, it still stirs the imagination when people think about Antarctica. Paradoxically, this has also limited our ability to fully exploit the unique conditions to conduct front-line science, as much of our thinking is mired by pre-conceptions gleaned from the survival tales of the heroic age. This has limited the ability of many nations to grasp the new opportunities now available. Overcoming human perceptions about the environment of the Antarctic plateau is proving to be at least as difficult as developing the new front line facilities themselves. With the start of the third millennium, and a growing knowledge and awareness of Antarctica, such perceptions are waning. The challenge for the astronomer now is to work out how to grasp the opportunity, to conduct science that might otherwise only be tackled from space. The Antarctic plateau provides sites where the ultimate Earth-based telescopes may be built, if we can find the way to build on the pioneering endeavours of the past two decades, and establish the necessary infrastructure development that can lead to new astronomical facilities.

Acknowledgements Astronomy in Antarctica has provided a peripatetic journey for me for nearly two decades, one with its full share of successes and failures, of amazing highs and deep lows, but always with a sense of discovery at the frontier. A great many people have provided inspiration and support along the way, on a path that has now found me trying to tell the factual story behind a new field of scientific endeavour in this review article. David Allen, Peter Gillingham and Russell Cannon each encouraged my youthful enthusiasm in the infant field while I worked at the Anglo Australian Observatory and John Storey gave me the opportunity to take my first steps in Antarctica with a job at UNSW. Together with Michael Ashley, the three of us stumbled through some early adventures at the South Pole. Al Harper and John Bally, and later John Carlstrom, made possible our participation in the early years of the CARA venture there, and this gave us the springboard to take our program to the high plateau. Our group has waxed and waned over the years, dictated by the vagaries of university funding, but with Jon Lawrence's joining our program managed to wind its way forward, from the Pole to Dome C, then Dome A and now towards Dome F, with even Ridge A now in our sights. All these colleagues have contributed immeasurably to Astronomy in Antarctica and many of the scientific highlights reported here. So too have our many support staff, postdoctoral fellows and research students, at UNSW, the AAO and other places around Australia. Several somehow managed to winter-over in Antarctica along the way, an opportunity I missed in my youth! Marc Duldig has provided invaluable insight into the mysterious workings of the Antarctic establishment. Our continually growing list of international colleagues, first in the USA, then France and Italy, extending around the European Union through the ARENA network, and now to China and Japan, are all contributing to the vitality of this field and the science that has been accomplished, as well as that which may one day be possible. The referee, Roland Gredel, also provided insightful comments and corrected several errors. But none of this would have happened for me without the support and understanding of my dear wife Connie, who could not have imagined what the past twenty years could possibly bring and where it would lead us to.

\section{References}

1. Ackermann M et al. (123 authors) (2005) Search for extraterrestrial point sources of high energy neutrinos with AMANDA-II using data collected in 2000-2002. Phy. Rev. D 71:077102

2. Agabi A, Aristidi E, Azouit M, Fossat E, Martin F, Sadibekova T, Vernin J, Ziad A (2006) First whole atmosphere nighttime seeing measurements at Dome C, Antarctica. PASP 118:344-348

3. Aharonian F et al. (98 authors) (2004) Calibration of the cameras of the HESS detector. Astroparticle Physics 22:109-125

4. Ahrens J et al. (131 authors) (2004) Status of the IceCube neutrino observatory. New Astronomy Reviews 48:519-525

5. Andrés E et al. (67 authors from the AMANDA Collaboration) (2000) The AMANDA neutrino telescope: principle of operation and first results. Astroparticle Physics 13:1-20

6. Andrés E et al. (119 authors from the AMANDA Collaboration) (2001) Observation of high energy neutrinos using Cherenkov detectors embedded deep in Antarctic ice. Nature 410:441-443 
7. Aristidi E, Agabi A, Vernin J, Azouit M, Martin F, Ziad A, Fossat E (2003) Antarctic site testing: first daytime seeing monitoring at Dome C. A\&A 406:L19-L22

8. Aristidi A, Agabi A, Fossat E, Azouit M, Martin F, Sadibekova T, Travouillon T, Vernin J, Ziad A (2005a) Site testing in summer at Dome C, Antarctica. A\&A 444:651-659

9. Aristidi A, Agabi K, Azouit M, Fossat E, Vernin J, Travouillon T, Lawrence JS, Meyer C, Storey JWV, Halter B, Roth WL, Walden V (2005b) An analysis of temperatures and wind speeds above Dome C, Antarctica. A\&A 430:739-746

10. Aristidi E, Fossat E, Agabi A, Mékarnia D, Jeanneaux E, Challita Z, Ziad A, Vernin J, Trinquet H (2009) Dome C site testing: surface layer, free atmospheric seeing and isoplanatic angle statistics. A\&A 499:955965

11. Ashley MCB, Burton MG, Calisse PG, Phillips A, Storey JWV (2005) Site testing at Dome C: cloud statistics from the ICECAM experiment. Highlights of Astronomy 13:932:934

12. Ashley MCB, Burton MG, Storey JWV, Lloyd JP, Bally J, Briggs JW, Harper DA (1996) South Pole observations of the near-infrared sky brightness. PASP 108:721-723

13. Askaryan GA (1965) Coherent radio emission from cosmic showers in air and in dense media. Soviet Physics JETP 21:658

14. Barwick SW et al. (18 authors) (2003) Overview of the ANITA project. SPIE 4858:265-276

15. Bayly PGW, Stillwell FL (1923) The Adelie Land meteorite. Scientific Reports Australasian Antarctic Expedition (1911-1914) Series A, Vol 4, Part 1 Geology

16. Bernasconi PN, Rust DM, Murphy GA, Eaton HAC (1999) High resolution polarimetry with a balloonborne telescope: the Flare Genesis experiment. In Rimmele TR, Balasubramaniam KS, Radick RR (eds) High resolution solar physics: theory, observations and techniques. Ast. Soc. Pacific conf. ser. 183:279-287

17. Bodhaine BA (1995) Aerosol absorption measurements at Barrow, Mauna Loa and the South Pole. Journal Geophysical Research 100:8967-8976

18. Bonner CS, Ashley MCB, Lawrence JS, Storey JWV, Luong-Van DM, Bradley SG (2009) SNODAR II: probing the atmospheric boundary layer on the Antarctic plateau. In Masciadri E, Sarazin M (eds) Optical turbulence: astronomy meets meteorology. Proc. Optical Turbulence Characterisation for Astronomical Applications, Sardinia, Italy, 15-18 September 2008

19. Bonner CS, Ashley MCB, Cui X, Feng L, Gong X, Lawrence JS, Luong-Van DM, Storey JWV, Wang L, Yang H, Yang J, Zhou X, Zhu Z (2010) Height of the atmospheric boundary layer above Dome A, Antarctica during 2009. PASP submitted.

20. Bromwich DH (1988) Snow in high southern latitudes. Reviews Geophysics 26:149-168

21. Brooks KJ, Burton MG, Rathborne JM, Ashley, MCB, Storey JWV (2000) Unlocking the Keyhole: $\mathrm{H}_{2}$ and PAH emission from molecular clumps in the Keyhole Nebula. MNRAS 319:95-102

22. Burova LP, Gromov VD, Luk'yanchikova NI, Sholomitskii GB (1986) Low humidity and submillimeter transparency above the Vostok Antarctic station. Soviet Astronomy Letters 12:339-342

23. Burton MG (2005) Astronomy in Antarctica. In Heck A (ed) Organisations and Strategies in Astronomy. Astrophysics \& Space Library, Kluwer 5:11-37

24. Burton MG et al. (20 authors) (1994) The scientific potential for astronomy from the Antarctic plateau. PASA $11: 127-150$

25. Burton MG et al. (27 authors) (2005) Science programs for a 2-m class telescope at Dome C, Antarctica: PILOT, the Pathfinder for an International Large Optical Telescope. PASA 22:199-235

26. Burton MG, Burgarella D, Andersen M, Busso M, Eiroa C, Epchtein N, Maillard J-P, Persi P (ARENA Working Group 1) (2010) A wide-field, optical/infrared, $2.5 \mathrm{~m}$ class telescope for Antarctica. In Spinoglio L, Epchtein N (eds) $3^{\text {rd }}$ ARENA Conference on an Astronomical Observatory at Concordia (Dome C, Antarctica). European Astronomical Soc. pub. ser. 40:125-135

27. Burton MG, Allen DA, McGregor P (1993) The potential of near-infrared astronomy in Antarctica. Aust. Inst. Physics $10^{\text {th }}$ Congress (Feb 1992). ANARE Research Notes 88:293-300

28. Burton MG, Ashley MCB, Marks RD, Schinckel AE, Storey JWV, Fowler A, Merrill M, Sharp N, Gatley I, Harper DA, Loewenstein RF, Mrozek F, Jackson JM Kraemer KE (2000) High resolution imaging of photodissociation regions in NGC 6334. ApJ 542:359-366

29. Burton MG, Storey JWV, Ashley MCB (2001) Science goals for Antarctic infrared telescopes. PASA 18:158-165

30. Burton MG (ed) (2005b) Highlights of Astronomy. Issue 13, pp927-976. IAU XXV General Assembly, Special Session 2: Astronomy in Antarctica, Sydney, July 18-19 2003. Series editor O Engvold. Ast. Soc. Pacific Conf. Series

31. Burton MG (ed) (2007) Highlights of Astronomy. Issue 14, Volume 2, pp683-712. IAU XXVI General Assembly, Special Session 7: Astronomy in Antarctica, Prague, August 22-23 2006. Series editor KA van der Hulcht. Cambridge University Press

32. Burton MG (ed) (2010b) Highlights of Astronomy. Issue 15, in press. IAU XXVII General Assembly, Special Session 3: Astronomy in Antarctica, Rio de Janeiro, August 6-7 2009. Series editor I Corbett. Cambridge University Press 
33. Bussmann RS, Holzapfel WL, Kuo CL (2005) Millimeter wavelength brightness fluctuations of the atmosphere above the South Pole. ApJ 622:1343-1355

34. Busso M et al. (10 authors) (2010) Science with the IRAIT telescope: the commissioning phase. In Spinoglio L, Epchtein N (eds) $3^{\text {rd }}$ ARENA Conference on an Astronomical Observatory at Concordia (Dome C, Antarctica). European Astronomical Soc. pub. ser. 40:165-170

35. Calisse P, Ashley MCB, Burton MG, Phillips MA, Storey JWV, Radford SJE, Peterson JB (2004) Submillimetre site testing at Dome C, Antarctica. PASA 21:256-263

36. Carlstrom JE et al. (40 authors) (2010) The 10 meter South Pole Telescope. PASP in press

37. Castro PH et al. (32 authors) (2009) Cosmological parameters from the QUAD CMB polarization experiment. ApJ 701:857-864

38. Chamberlain, MA, Ashley MCB, Burton MG, Phillips A Storey JWV (2000) Mid-infrared observing conditions at the South Pole. ApJ 535:501-511

39. Chamberlin RA, Lane AP, Stark AA (1997) The $492 \mathrm{GHz}$ atmospheric opacity at the geographic South Pole. ApJ 476:428-433

40. Chiang HC et al. (30 authors) (2010) Measurement of cosmic microwave background polarization power spectra from two years of BICEP data. ApJ 711:1123-1140

41. Coble K et al. (15 authors) (1999) Anisotropy in the cosmic microwave background at degree angular scales: Python V results. ApJ 519:L5-L8

42. Crouzet $\mathrm{N}$ et al. (22 authors) (2010) ASTEP South: an Antarctic search for transiting exoplanets around the celestial South Pole. A\&A 511:A36

43. Cui X (2010) CSTAR and future plans for Dome A. Highlights of Astronomy 15: in press

44. Culverhouse T et al. (31 authors) (2010) The QUaD galactic plane survey 1: maps and analysis of diffuse emission. ApJ submitted

45. Damé L, Andretta V and ARENA Solar Astrophysics working group members (2010) ARENA solar astrophysics working group reporting on Dome C exceptional potential for solar observations. In Spinoglio L, Epchtein N (eds) $3^{\text {rd }}$ ARENA Conference on an Astronomical Observatory at Concordia (Dome C, Antarctica). European Astronomical Soc. pub. ser. 40:451-466

46. de Bernardis P. et al. (36 authors) (2000) A flat Universe from high-resolution maps of the cosmic microwave background radiation. Nature 404:955-959

47. Dempsey JT, Storey JWV, Phillips A (2005) Auroral contribution to sky brightness for optical astronomy on the Antarctic plateau. PASA 22:91-104

48. Devlin M et al. (29 authors) (2009) Over half of the far-infrared background light comes from galaxies at $z \geq 1.2$. Nature $458: 737-739$

49. Dickinson JE, Gill JR, Hart SP, Hill GC, Hinton JA, Lloyd-Evans J, Potter D, Pryke C, Rochester K, Schwarz R, Watson AA (2000) A new air-Cherenkov array at the South Pole. Nuclear Instruments and Methods in Physics Research A 440:114-123

50. Dolci M et al. (19 authors) (2010) Status of the AMICA project: ready for Antarctic adventure. In Spinoglio L, Epchtein N (eds) $3^{\text {rd }}$ ARENA Conference on an Astronomical Observatory at Concordia (Dome C, Antarctica). European Astronomical Soc. pub. ser. 40:171-176

51. Duldig M (2002) Cosmic ray physics and astronomy. In Marchant HJ, Lugg DJ \& Quilty PG (eds) Australian Antarctic Science: the first 50 years of ANARE. Published by Australian Antarctic Division pp43-71

52. Epchtein N (ed) for the ARENA consortium (2010) A vision for European astronomy and astrophysics at the Antarctic station Concordia, Dome C. Prepared by Antarctic Research, a European Network for Astrophysics (ARENA) for EC-FP6 contract RICA 026150

53. Epchtein N, Candidi M (eds) (2007) $1^{\text {st }}$ ARENA conference on large astronomical infrastructure at Concordia, prospects and constraints for Antarctic optical/IR astronomy. European Astronomical Soc. pub. ser. Vol 40

54. Filimonov K and the IceCube consortium (2010) IceCube neutrino observatory at the South Pole: recent results. Highlights of Astronomy 15: in press

55. Fossat E, Candidi M (2003) The scientific outlook for astronomy and astrophysics research at the Concordia station. Mem. della Soc. Ast. Italiana Vol 2

56. Fossat E (2005) The Concordia Station on the Antarctic plateau: the best site on Earth for the 21st century astronomers. J. Astrophysics \& Astronomy 26:349-357

57. Fowler A et al. (11 authors) (1998) Abu/SPIREX: South Pole thermal IR experiment. SPIE 3354:11701178

58. Gabriel A et al. (32 authors) (1995) Global oscillations at low frequency from the SOHO mission (GOLF). Solar Physics 162:61-99

59. Gillingham PR (1989) Antarctic optical-infrared observatory, presented to a meeting on the future of Australian astronomy, organised by the Ast. Soc. Aust, Canberra, June 1989 
60. Gillingham P (ed) (1992) Highlights of Astronomy. Issue 9, pp577-602. IAU XXI General Assembly, Buenos Aires, July 1991. Series editor J Bergeron. Kluwer

61. Gillingham PR (1993) Antarctic astronomy: introduction and summary of international developments affecting Australia. Australian Institute Physics 10th Congress Melbourne (Feb. 1992), ANARE Research Notes 88:290-292

62. Gong et al. (37 authors) (2010) Dome A site testing and future plans. In Spinoglio L, Epchtein N (eds) $3^{\text {rd }}$ ARENA Conference on an Astronomical Observatory at Concordia (Dome C, Antarctica). European Astronomical Soc. pub. ser. 40:65-72

63. Gorham PW et al. (43 authors) (2009) New limits on the ultra-high energy cosmic neutrino flux from the ANITA experiment. Phy. Rev. Lett. 103:051103

64. Gorham PW et al. (37 authors) (2010) Observational constraints on the ultra-high energy cosmic neutrino flux from the second flight of the ANITA experiment. Phy. Rev. Lett. submitted

65. Grec G, Fossat E, Pomerantz M (1980) Solar oscillations: full disk observations from the geographic South Pole. Nature 288:541-544

66. Grec G, Fossat E, Pomerantz M (1983) Full-disk observations of solar oscillations from the geographic South Pole: latest results. Solar Physics 82:55-66

67. Gredel R (2010) Site characterisation at Dome C: the ARENA work. In Spinoglio L, Epchtein N (eds) $3^{\text {rd }}$ ARENA Conference on an Astronomical Observatory at Concordia (Dome C, Antarctica). European Astronomical Soc. pub. ser. 40:11-20

68. Harper DA (1990) Infrared astronomy in Antarctica. In Pomerantz, M (ed) Astrophysics in Antarctica. American Institute Physics conf. series 198:123-129

69. Hereld M (1994) SPIREX: near infrared astronomy from the South Pole. In McLean IS (ed) Infrared astronomy with arrays, the next generation. Astrophysics and Space Science Library 190:248-252

70. Hereld M, Rauscher BJ, Harper DA, Pernic RJ (1990) GRIM: a near-infrared grism spectrometer and imager. SPIE Instrumentation in Astronomy VII pp43-48

71. Hidas MG, Burton MG, Chamberlain MA, Storey JWV (2000) Infrared and sub-millimetre observing conditions on the Antarctic plateau. Pub. Ast. Soc. Aust. 17:260-269

72. Ichikawa T (2010) Future plans for astronomy at Dome Fuji. Highlights of Astronomy 15: in press

73. Indermuehle BT, Burton MG, Maddison ST (2005) The history of astrophysics in Antarctica. PASA 2005:73-90

74. Ishii S, Seta M, Nakai N, Nagai S, Miyagawa N, Yamauchi A, Motoyama H, Taguchi M (2010) Site testing at Dome Fuji for sub-millimeter and terahertz astronomy: $220 \mathrm{GHz}$ atmospheric transparency. Polar Science 3:213-221

75. Jackson JM, Gatley I, Bania TM, Tollestrup E, Dunham T (2001) The Antarctic Infrared Observatory AIRO. Bulletin American Astro. Soc. 33:1466

76. Kennedy JR and the GONG Team (1994) GONG, a global network of automated solar telescopes. In Pyper DM, Angione RJ (eds) Optical Astronomy from the Earth and Moon. Ast. Soc. Pacific conf. ser. 55:188-196

77. Kenyon SJ, Gomez M (2001) A $3 \mu \mathrm{m}$ survey of the Chamaeleon I dark cloud. AJ 121:2673-2680

78. Kenyon SL, Lawrence JS, Ashley MCB, Storey JWV, Tokovinin A, Fossat E (2006) Atmospheric scintillation at Dome C, Antarctica: implications for photometry and astrometry. 118:924-932

79. Kenyon SL, Storey JWV (2006) A review of optical sky brightness and extinction at Dome C, Antarctica. PASP 118:489-502

80. Kim S, Narayanan D (2006) [CI] $809 \mathrm{GHz}$ imaging of the NGC 6334 complex. PASJ 58:753-757

81. Kovac JM, Leitch EM, Pryke C, Carlstrom JE, Halverson NW, Holzapfel WL (2002) Detection of polarization in the cosmic microwave background using DASI. Nature 420:772-787

82. Kravchenko I et al. (14 authors) (2008) Status of the RICE experiment. In Caballero R et al. (eds) Proc. $30^{\text {th }}$ Intl. Cosmic Ray Conf. Yucatán Mexico 3:1229-1232

83. Kulesa C, Hungerford AL, Walker CK, Zhang X, Lane AP (2005) Large scale CO and [CI] emission in the $\rho$ Ophiuchi molecular cloud. ApJ 625:194-209

84. Kuo CL et al. (14 authors) (2004) High-resolution observations of the cosmic microwave background power spectrum with ACBAR. ApJ 600:32-51

85. Lane AP (1998) Submillimeter transmission at South Pole. In Novak G, Landsberg RH (eds) Astrophysics from Antarctica ASP Conf. Ser. 141:289-295

86. Lascaux F, Masciadri E, Hagelin S, Stoesz J (2009) Mesoscale optical turbulence simulations at Dome C. MNRAS 398:1093-1104

87. Lascaux F, Masciadri E, Hagelin S (2010) Mesoscale optical turbulence simulations at Dome C: refinements. MNRAS 403:1714-1718

88. Lawrence JS (2004) Infrared and sub-millimeter atmospheric characteristics of high Antarctic plateau sites. PASP 116:482-492 
89. Lawrence JS, Ashley MCB, Hengst S, Luong-Van DM, Storey JWV, Yang H, Zhou X \& Zhu, Z (2009) The PLATO Dome A site-testing observatory: power generation and control systems. Rev. Sci. Inst. 80:064501:1-10

90. Lawrence JS, Ashley MCB, Storey JWV (2005) A remote, autonomous laboratory for optical astronomy on the Antarctic plateau. Aust. Journal Electrical \& Electronic Engineering 2:1-12

91. Lawrence JS, Ashley MCB, Tokovinin A, Travouillon T (2004) Exceptional astronomical seeing conditions above Dome C in Antarctica. Nature 431:278-281

92. Lawrence JS et al. (43 authors) (2009a) The science case for PILOT I: summary and overview. PASA 26:379-396

93. Lawrence JS et al. (13 authors) (2009b) The science case for PILOT II: the distant universe. PASA 26:397-414

94. Lawrence JS et al. (23 authors) (2009c) The science case for PILOT III: the nearby universe. PASA 26:415-438

95. Leitch EM, Kovac JM, Pryke C, Carlstrom JE, Halverson NW, Holzapfel WL, Dragovan M, Reddall B, Sandberg ES (2002) Measurement of polarization with the Degree Angular Scale Interferometer. Nature 420:763-771

96. Li H, Griffin GS, Krejny M, Novak G, Loewenstein RF, Newcomb MG, Calisse PG, Chuss DT (2006) Results of SPARO 2003: mapping magnetic fields in giant molecular clouds. ApJ 648:340-354

97. Lloyd JP, Oppenheimer BR, Graham JR (2002) The potential of differential astrometric interferometry from the high Antarctic plateau. Pub. Ast. Soc. Aust. 19:318-322

98. Lloyd JP, Lane BF, Swain MR, Storey JWV, Travouillon T, Traub WA, Walker CK (2004) Extrasolar planet science with the Antarctic planet interferometer. SPIE 5170:193-199

99. Loewenstein RF, Bero C, Lloyd JP, Mrozek F, Bally J, Theil D (1998) Astronomical seeing at the South Pole. In Novak G, Landsberg R (eds) Astrophysics from Antarctica. ASP Conf. Series 141:296-302

100. Lueker M. et al. (43 authors) (2010) Measurements of secondary cosmic microwave background anisotropies with the South Pole Telescope. ApJ submitted

101. Lyo AR, Lawson WA, Mamajek EE, Feigelson ED, Sung EO, Crause LA (2003) Infrared study of the $\eta$ Chamaeleontis cluster and the longevity of circumstellar disks. MNRAS 338:616-622

102. Maercker M, Burton MG (2005) L-band (3.5 $\mu \mathrm{m})$ IR-excess in massive star formation. I: 30 Doradus. A\&A 438:663-673

103. Maercker M, Burton MG, Wright CM (2006) L-band (3.5 $\mu \mathrm{m})$ IR-excess in massive star formation. II: RCW57/NGC3576. A\&A 450:253-263

104. Marks RD (2002) Astronomical seeing from the summits of the Antarctic plateau. A\&A 385:328-336

105. Marks RD (2005) Antarctic site testing: measurement of optical seeing at the South Pole. PhD Thesis, University of New South Wales

106. Marks RD, Vernin J, Azouit M, Briggs JW, Burton MG, Ashley MCB, Manigault JF (1996) Antarctic site testing: microthermal measurements of surface-layer seeing at the South Pole. 118: 385-390

107. Marks RD, Vernin J, Azouit M, Manigault JF, Clevelin C (1999) Measurement of optical seeing on the high Antarctic plateau. A\&A Supp. 134:161-172

108. Marsden G et al. (38 authors) (2008) The Balloon-borne Large-Aperture Submillimeter Telescope for polarization: BLAST-pol. (2008) SPIE 7020:702002:1-12

109. Martin CL, Walsh WM, Xiao K, Lane AP, Walker CK Stark AA (2004) The AST/RO survey of the Galactic Centre region I: the inner 3 degrees. ApJS 150:239-262

110. Masi S et al. (44 authors) (2006) Instrument, method, brightness and polarization maps from the 2003 flight of BOOMERanG. A\&A 458:687-716

111. McCracken KG (1962) The cosmic-ray flare effect 3: deductions regarding the interplanetary magnetic field. Journal Geophysical Research 67:447-458

112. McGrath A, Saunders W, Gillingham P, Ward D, Storey J, Lawrence J, Haynes R (2008) Running PILOT: operational challenges and plans for an Antarctic observatory. SPIE 7016:70160G:1-12

113. McKay DS, Gibson EK, Thomas-Keprta KL, Vali H, Romanek C, Clemmett SJ, Chillier XDF, Maechling CR, Zare RN (1996) Search for past life on Mars: possible relic biogenic activity in Martian meteorite ALH84001. Science 273:924-930

114. Moore A et al. (49 authors) (2008a) Gattini: a multi-site campaign for the measurement of sky brightness in Antarctica. SPIE 7012:701226:1-10

115. Moore AM, Martin C, Maitless NC, Travouillon T (2008b) ACWI: an experiment to image the Cosmic Web from Antarctica. SPIE 7012:70122A:1-11

116. Morse R, Gaidos J (1990) A South Pole facility to observe very high energy gamma ray sources. In Pomerantz, M (ed) Astrophysics in Antarctica. American Inst. Physics conf. series 198:24-34

117. Mosser B, Aristidi E (2007) Duty cycle of Doppler ground-based asteroseismic observations. 119:127133 
118. Motizuki Y, Takahashi K, Makishima K, Bamba A, Nakai Y, Yano Y, Igarashi M, Motoyama H, Kamiyama K, Suzuki K, Imamura, T (2010) An Antarctic ice core recording of both supernovae and solar cycles. Nature submitted

119. Nagata T (ed) (1975) Yamato meteorites collected in Antarctic in 1969. Memoirs National Polar Institute Polar Research, Special Issue 5

120. Netterfield CB et al. (27 authors) (2009) BLAST: the mass function, lifetimes and properties of intermediate mass cores from a 50 square degree sub-millimetre galactic plane survey in Vela $l \sim 265^{\circ}$. ApJ 707:1824-1835

121. Nguyen HT, Rauscher BJ Severson SA, Hereld M, Harper DA, Loewenstein RF, Mrozek F, Pernic RJ (1996) The South Pole near-infrared sky brightness. PASP 108:718-720

122. Novak G, Landsberg RH (eds) (1998) Astrophysics from Antarctica. Astron. Soc. Pacific Conf. Series 141

123. Novak G, Chuss DT, Renbarger T, Griffin GS, Newcomb MG, Peterson JB, Loewenstein RF, Pernic D, Dotson JL (2003) First results from the Sub-millimeter Polarimeter for Antarctic Remote Observations (SPARO): evidence of large-scale toroidal magnetic fields in the Galactic Center. ApJL 583:L83-L86

124. Oberst TE, Parshley SC, Stacey GJ, Nikola T, Löhr A, Harnett JI, Tothill NFH, Lane AP, Stark AA, Tucker CE (2006) Detection of the $205 \mu \mathrm{m}$ [NII] line from the Carina Nebula. ApJL 652:L125-L128

125. Olmi L et al. (37 authors) (2009) The BLAST survey of the Vela Molecular Cloud: physical properties of the dense cores in Vela-D. ApJ 707:1836-1851

126. Parsons NR (1957) Directional measurements of the daily variation of cosmic ray meson intensity at $\lambda=73^{\circ}$ S. Aust. J. Phys. 10:462-470

127. Pascale E et al. (30 authors) (2008) The Balloon-borne Large Aperture Submillimeter Telescope: BLAST. ApJ 681:400-414

128. Phillips A, Burton MG, Ashley MCB, Storey JWV, Lloyd JP, Harper DA, Bally J (1999) The nearinfrared sky emission at the South Pole in winter. ApJ 527:1009-1022

129. Peterson JB, Griffith GS, Newcomb MG, Alvarez DL, Cantalupo CM, Morgan D, Miller KW, Ganga K, Pernic D, Thoma M (2000) First results from Viper: detection of small-scale anisotropy at $40 \mathrm{GHz}$. ApJ 532:L83-L86

130. Peterson JB, Radford SJE, Ade PAR, Chamberlin RA, O'Kelly MJ, Peterson KM, Schartman E (2003) Stability of the sub-millimeter brightness of the atmosphere above Mauna Kea, Chajnantor and the South Pole. PASP 115:383-388

131. Plagge T et al. (45 authors) (2010) Sunyaev-Zel'dovich cluster profiles measured with the South Pole Telescope. ApJ submitted

132. Pomerantz MA, Agarwal SP, Pontis VR (1958) Direct observation of periodic variation of primary cosmic-ray intensity. Phys. Rev. 109:224-225

133. Pomerantz M (ed) (1990) Astrophysics in Antarctica. American Inst. Physics conf. series 198

134. Rathborne JM, Burton MG (2005) Results from the South Pole Infra-Red EXplorer Telescope. Highlights of Astronomy 13:937-944

135. Rathborne JM, Burton MG, Brooks KJ, Cohen M, Ashley MCB, Storey JWV (2002) Photodissociation regions and star formation in the Carina Nebula. MNRAS 331:85-97

136. Saunders W, Gillingham P, McGrath A, Haynes R, Brzeski J, Storey J, Lawrence J (2008a) PILOT: a wide-field telescope for the Antarctic plateau. SPIE 7012:70124F:1-9

137. Saunders W, Gillingham P, McGrath A, Haynes R, Storey J, Lawrence J, Burton M, Jenkins C, Alcione M (2008b) Proposed instrumentation for PILOT. SPIE 7014:70144N:1-9

138. Saunders W, Lawrence JS, Storey JWV, Ashley MCB, Kato S, Minnis P, Winker DM, Liu G, Kulesa C (2009) Where is the best site on Earth? Domes A, B, C and F, and Ridges A and B. PASP 121:976-992

139. Severson S (2000) Death of a comet: SPIREX observations of the collision of SL9 with Jupiter. PhD Dissertation, University of Chicago

140. Siebenmorgen R (2010) Thermal infrared instruments for Antarctica: what can be gained. In Spinoglio L, Epchtein N (eds) $3^{\text {rd }}$ ARENA Conference on an Astronomical Observatory at Concordia (Dome C, Antarctica). European Astronomical Soc. pub. ser. 40:147-155

141. Smith CH, Harper DA (1998) Mid-infrared sky brightness site testing at the South Pole. PASP 110:747753

142. Smith NJT, Gaisser TK, Hillas AM, Ogden PA, Patel M, Perrett JC, Pomerantz MA, Reid RJO, Stanev T, Watson AA (1989) The South Pole Air Shower Experiment. In Stepanian AA, Fegan DJ, Cawley MF (eds) Very High Energy Gamma Ray Astronomy. Proc. Workshop Crimea, 17-21 April 1988 pp55

143. Smythe WD, Jackson BV (1977) Atmospheric water vapor at South Pole. Applied Optics 16:2041-2042

144. Spergel DN et al. (17 authors) (2003) First-year Wilkinson Microwave Anisotropy Probe (WMAP) observations: determination of cosmological parameters. ApJS 148:175-194

145. Spinoglio L, Epchtein N (eds) (2010) $3^{\text {rd }}$ ARENA conference on an astronomical observatory at Concordia (Dome C, Antarctica). European Astronomical Soc. pub. ser. Vol 40 
146. Staniszewski Z et al. (44 authors) (2009) Galaxy clusters discovered with a Sunyaev-Zel'dovich effect survey. ApJ 701:32-41

147. Stark AA, Bolatto AD, Chamberlin RA, Lane AP, Bania TM, Jackson JM, Lo KY (1997) First detection of $492 \mathrm{GHz}[\mathrm{CI}]$ emission from the Large Magellanic Cloud. ApJL 480:L59-L62

148. Stark AA et al. (27 authors) (2001) The Antarctic Sub-millimeter Telescope and Remote Observatory (AST/RO). PASP 113:567-585

149. Storey JWV (2004) Antarctica: the potential for interferometry. SPIE 5491:169-175

150. Storey JWV (2009) Astronomy and Astrophysics from Antarctica. Association Asia Pacific Physical Societies Bulletin. 19:4-10

151. Storey JWV (2010) Astronomy and Astrophysics from Antarctica: a new SCAR Scientific Research Program. Highlights of Astronomy 15: in press

152. Storey JWV, Ashley MCB, Burton MG (1996) An automated astrophysical observatory for Antarctica. PASA 13:35-38

153. Storey JWV, Ashley MCB, Burton MG, Lawrence JS (2007) PILOT - the pathfinder for an international large optical telescope. In Epchtein N, Candidi M (eds) $1^{\text {st }}$ ARENA conference on large astronomical infrastructures at Concordia, prospects and constraints for Antarctic optical/IR astronomy. European Astronomical Soc. pub. ser. 25:255-259

154. Storey JWV, Ashley MCB, Lawrence JS, Burton MG (2003) Dome C-the best astronomical site in the world? Mem. S. A. It. Supp. 2:13-18

155. Storey JWV (2005) Astronomy from Antarctica. Antarctic Science 17:555-560

156. Strassmeier K et al. (14 authors) (2008) First time-series optical photometry from Antarctica. sIRAIT monitoring of the RS CVn binary V841 Centauri and the $\delta$-Scuti star V1034 Centauri. A\&A 490:287-295

157. Takahashi Y et al. (26 authors) (2010) Characterisation of the BICEP telescope for high-precision cosmic microwave background polarimetry. ApJ 711:1141-1156

158. Takato N, Ichikawa T, Uraguchi F, Lundock R, Murata C, Taniguchi Y, Motoyama H, Fukui K, Taguchi M (2008) A 2 m class telescope at Dome Fuji. In Zinnecker H, Epchtein N, Rauer H (eds) $2^{\text {nd }}$ ARENA conference on the astrophysical science cases at Dome C. European Astronomical Soc. pub. ser. 33:271274

159. Taylor MJ (1990) Photometry of the $4686 \AA$ emission line of Gamma 2 Velorum from the South Pole. AJ 100:1264-1269

160. Tomasi C, Petkov B, Benedetti E, Valenziano L, Lupi A, Vitale V, Bonafé U (2008) A refined calibration procedure of two-channel sun photometers to measure atmospheric precipitable water at various Antarctic sites. J. Atmospheric \& Oceanic Technology, 25:213-229

161. Townes GH, Melnick G (1990) Atmospheric transmission in the far-infrared at the South Pole and astronomical applications. PASP 102:357-367.

162. Travouillon T (2004) Measurements of optical turbulence on the Antarctic plateau and their impact on astronomical observations. PhD Thesis, University of New South Wales

163. Travouillon T, Ashley MCB, Burton MG, Storey JWV, Loewenstein RF (2003a) Atmospheric turbulence at the South Pole and its implications for astronomy. A\&A 400:1163-1172

164. Travouillon T, Ashley MCB, Burton MG, Lawrence J, Storey JWV (2003b) Low atmospheric turbulence at Dome C: preliminary results. Mem. Soc. Astron. Italy 2:150-153

165. Trinquet H, Agabi A, Vernin J, Azouit M, Aristidi E, Fossat E (2008) Nighttime optical turbulence vertical structure above Dome C in Antarctica. PASP 120:203-211

166. Tucker GS, Griffin GS, Nguyen HT, Peterson JB (1993) A search for small-scale anisotropy in the cosmic microwave background. ApJL 419:L45-L48

167. Valenziano L, dall'Oglio G (1999) Millimetre astronomy from the high Antarctic plateau: site testing at Dome C. PASA 16:167-174

168. van Stekelenborg J, Gaisser TK, Perrett JC, Petrakis JP, Stanev TS, Beaman J, Hillas AM, Johnson PA, Lloyd-Evans J, Smith NJT, Watson AA (1993) Search for point sources of ultra-high energy $\gamma$-rays in the southern hemisphere with the South Pole Air Shower Experiment. Phy. Rev D 48:4504-4517

169. Vernin J, Chadid M, Aristidi E, Agabi A, Trinquet H, van der Swaelmen M (2009) First single star scidar measurement at Dome C, Antarctica. A\&A 500:1271-1276

170. Walden VP, Town MS, Halter B, Storey JWV (2005) First measurements of the infrared sky brightness at Dome C, Antarctica. PASP 117:300-308

171. Wales W, Bayly W (1777) The original astronomical observations made in the course of a voyage towards the South Pole and round the world in his majesty's ships the Resolution and the Adventure in the years 1772-1775. Publishers Board of Longitude. Printers WA Strahan, London.

172. Walker CK et al. (24 authors) (2008) The Stratospheric Terahertz Observatory (STO): an LDB experiment to investigate the life cycle of the interstellar medium. In $19^{\text {th }}$ Intl. Symp. on Space Terahertz Technology. Grongingen pp28-31 
173. Windhorst RA et al. (10 authors) (1991) The discovery of a young radio galaxy at $z=2.390$ - probing initial star formation at $z$ less than approximately 3.0. ApJ 380:362-383

174. Yang H, Kulesa CA, Walker CK, Tothill NFH, Yang J, Ashley MCB, Cui X, Feng L, Lawrence JS, Luong-Van DM, Storey JWV, Wang L, Zhou X, Zhu Z (2010) Exceptional terahertz transparency and stability above Dome A, Antarctica. PASP 122:490-494

175. Yang H et al. (37 authors) (2009) The PLATO Dome A site-testing observatory: instrumentation and first results. PASP 121:174-184

176. Zhang X, Lee Y, Bolatto AP, Stark AA (2001) CO 4-3 and [CI] observations of the Carina molecular cloud complex. ApJ 553:274-287

177. Zhao G-B, Zhan H, Wang L, Fan Z, Zhang X (2010) Probing dark energy with the Kunlun Dark Universe Survey Telescope. ApJ submitted

178. Zhou X et al. (32 authors) (2010) The first release of the CSTAR point source catalog from Dome A, Antarctica. PASP 122:347-353

179. Ziad A, Aristidi E, Agabi A, Borgino J, Martin F, Fossat E (2008) First statistics of the turbulence outer scale at Dome C. A\&A 491:917-921

180. Zinnecker H, Epchtein N, Rauer H (eds) (2008) $2^{\text {nd }}$ ARENA conference on the astrophysical science cases at Dome C. European Astronomical Soc. pub. ser. Vol 33

181. Zou $\mathrm{H}$ et al. (34 authors) (2010) The sky brightness and transparency in $i$-band at Dome A, Antarctica. AJ submitted 